\title{
Combined Mean-Field and Semiclassical Limits of Large Fermionic Systems
}

\author{
Li Chen ${ }^{1} \cdot$ Jinyeop Lee ${ }^{2} \cdot$ Matthew Liew ${ }^{1}$ (D)
}

Received: 29 October 2019 / Accepted: 9 January 2021 / Published online: 25 January 2021

(c) The Author(s) 2021

\section{Abstract}

We study the time dependent Schrödinger equation for large spinless fermions with the semiclassical scale $\hbar=N^{-1 / 3}$ in three dimensions. By using the Husimi measure defined by coherent states, we rewrite the Schrödinger equation into a BBGKY type of hierarchy for the $k$ particle Husimi measure. Further estimates are derived to obtain the weak compactness of the Husimi measure, and in addition uniform estimates for the remainder terms in the hierarchy are derived in order to show that in the semiclassical regime the weak limit of the Husimi measure is exactly the solution of the Vlasov equation.

Keywords Large fermionic system · Husimi measure - Semiclassical limit · BBGKY . Wasserstein distance $\cdot$ Vlasov equation

\section{Introduction}

In this paper, we aim to study the combined mean-field and semiclassical limit of $N$-fermions from time-dependent Schrödinger equation to Vlasov equation.

The following anti-symmetric subspace of $L^{2}\left(\mathbb{R}^{3 N}\right)$ is considered for fermions,

$$
L_{a}^{2}\left(\mathbb{R}^{3 N}\right):=\left\{\Psi \in L^{2}\left(\mathbb{R}^{3 N}\right): \Psi\left(q_{\pi(1)}, \ldots, q_{\pi(N)}\right)=\varepsilon(\pi) \Psi\left(q_{1}, \ldots, q_{N}\right)\right\} .
$$

It is known that a system of fermions initially confined in a volume of order one have kinetic energy of order $N^{5 / 3}$ due to the Pauli principle. Therefore, to balance the order, the scale of the interaction term should be of order $N^{-1 / 3}$, we refer to $[6,8]$ for more details about this

Communicated by Eric A. Carlen.

$\triangle$ Matthew Liew

mliew@mail.uni-mannheim.de

Li Chen

chen@math.uni-mannheim.de

Jinyeop Lee

jinyeoplee@kias.re.kr

1 Department of Mathematics, University of Mannheim, 68131 Mannheim, Germany

2 School of Mathematics, Korea Institute for Advanced Study, Seoul 02455, Republic of Korea 
scaling. After a time rescaling of $N^{1 / 3}$ the Schödinger equation for $N$-fermions is written into

$$
N^{\frac{1}{3}} \mathrm{i} \partial_{t} \Psi_{N, t}=\left[-\frac{1}{2} \sum_{j=1}^{N} \Delta_{q_{j}}+\frac{1}{2 N^{\frac{1}{3}}} \sum_{i \neq j}^{N} V\left(q_{i}-q_{j}\right)\right] \Psi_{N, t} .
$$

By denoting the semiclassical scale $\hbar=N^{-1 / 3}$ and multiplying both sides by $\hbar^{2}$, one can recover the $N^{-1}$, the coupling constant for the mean field interaction. Hence one arrives at the following many body Schrödinger equation

$$
\left\{\begin{array}{l}
\mathrm{i} \hbar \partial_{t} \Psi_{N, t}=\left[-\frac{\hbar^{2}}{2} \sum_{j=1}^{N} \Delta_{q_{j}}+\frac{1}{2 N} \sum_{i \neq j}^{N} V\left(q_{i}-q_{j}\right)\right] \Psi_{N, t}=: H_{N} \Psi_{N, t}, \\
\Psi_{N, 0}=\Psi_{N},
\end{array}\right.
$$

where $\Psi_{N, t} \in L_{a}^{2}\left(\mathbb{R}^{3 N}\right), \Psi_{N}$ is the initial data in $L_{a}^{2}\left(\mathbb{R}^{3 N}\right)$, and $V$ is the interacting potential.

The limit from many body Schrödinger equation to the Vlasov equation has been studied extensively in the literature. Narnhofer and Sewell [34] and Spohn [46] are the first to prove this limit with the potential $V$ assumed to be analytic and $C^{2}$ respectively.

For large $N$, in the mean field limit regime, the solution of many body fermionic Schrödinger equation can be approximated by the solution of the following nonlinear HartreeFock equation,

$$
\left\{\begin{array}{l}
\mathrm{i} \hbar \partial_{t} \omega_{N, t}=\left[-\hbar^{2} \Delta+\left(V * \varrho_{t}\right)-X_{t}, \omega_{N, t}\right], \\
\omega_{N, 0}=\omega_{N},
\end{array}\right.
$$

where $\omega_{N, t}$ is the one-particle density matrix, $\varrho_{t}(q)=N^{-1} \omega_{N, t}(q ; q)$ and $X_{N, t}$ is a small term having the kernel $X_{t}(x, y)=N^{-1} V(x-y) \omega_{N, t}(x ; y)$. In [16], for the initial data being a Slater determinant, the approximation has been proved for short time for analytic interaction potential by using BBGKY hierarchy, while [6] proved the approximation with convergence rate for arbitrary time and weakened potential in the framework of second quantization. Similar results have been extended for mixed states in [4] and for relativistic case in [7]. Recently, with the help of Fefferman-de la Llave decomposition [18,26], weaker assumptions on the interaction potential have been considered. Specifically, Coulomb potential has been considered in [38], inverse power law in [41]. Further relevant literature on the fermionic case for the mean-field limit problem of Schrödinger equation can be found in [3,20,35-37].

In parallel, the mean field limit for the bosonic case from many body Schrödinger system to nonlinear Hartree equation was proved in [17] for Coulomb potential. Also for Coulomb potential, the convergence with rate $N^{1 / 2}$ has been obtained in [40]. Later, it has been optimized to the optimal convergence rate $N^{-1}$ in [11], and furthermore for stronger singular potentials in [10].

The semiclassical limit from Hartree-Fock equation to Vlasov equation has been obtained in the literature by using Wigner-Weyl transformation of the one-particle density matrix $\omega_{N, t}$ defined by

$$
W_{N, t}(q, p)=\left(\frac{\hbar}{2 \pi}\right)^{3} \int \mathrm{d} y e^{-i p \cdot y} \omega_{N, t}\left(x+\frac{\hbar}{2} y ; x-\frac{\hbar}{2} y\right),
$$

which has been intensively studied in the semiclassical limit of quantum mechanics by Lions and Paul in [31]. In [5] the authors compared the inverse Wigner transform of the Vlasov solution and the solution of Hartree-Fock and get the convergence rate in the trace norm as well as Hilbert-Schmidt norm with the regular assumptions on the initial data. The works in 


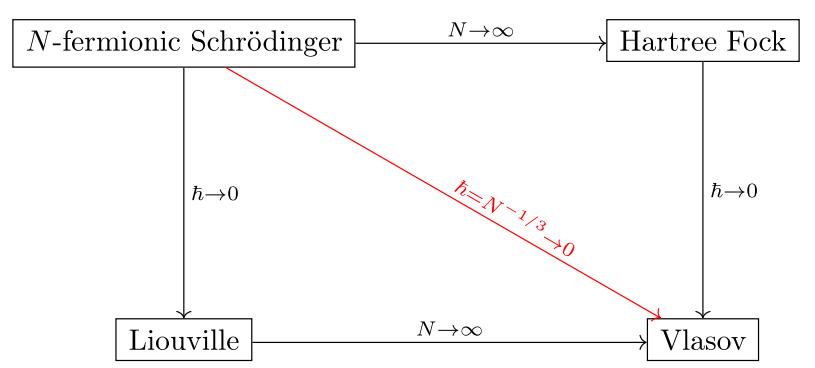

Fig. 1 Relations of $N$-fermionic Schrödinger systems to other mean-field equations [22,23]

this direction have also been extended for inverse power law potential [43], convergence rate in Schatten norm in [30], and Coulomb potential and mixed states in [42]. The convergence of relativistic Hartree dynamic to relativistic Vlasov equation has also been considered in [14]. Further convergence results from Hartree to Vlasov can be found in [1,2,21,33].

It is known that Wigner transform (1.2) is not a true probability density as it may be negative in certain phase-space. In fact, $[27,32,45]$ concludes that the Wigner measure is non-negative if and only if the pure quantum states are Gaussian, whilst [9] state that the Wigner measure is non-negative if the state is a convex combination of coherent states. Nevertheless, it has been shown that if one convolutes the Wigner measure with a Gaussian function in phase-space, it will yield a non-negative probability measure known as Husimi measure $[19,39,48]$. In fact, from [19, p.21], the Husimi measure is given by

$$
m_{N, t}^{(k)}=\frac{N(N-1) \cdots(N-k+1)}{N^{k}} W_{N, t}^{(k)} * \mathcal{G}^{\hbar},
$$

where $1 \leq k \leq N, \mathcal{G}^{\hbar}=(\pi \hbar)^{-3 k} \exp \left(-\hbar^{-1}\left(\sum_{j=1}^{k}\left|q_{j}\right|^{2}+\left|p_{j}\right|^{2}\right)\right)$ and $W_{N, t}^{(k)}$ is the Wigner transform of $k$-particle density matrix.

In the recent development, the convergence to Vlasov equation in the semiclassical Wasserstein pseudo-distance has been proved in [23-25,28,29]. The semiclassical Wasserstein pseudo-distance is computed between the Husimi measure and Vlasov solution.

One can also show the combined limit by first taking the semiclassical limit and then the mean field limit from many particle Schrödinger to Vlasov via the Liouville equations, and the corresponding BBGKY hierarchy. ${ }^{1}$ This has been done in [23]

Our goal, therefore, is to obtain the Vlasov equation from Schrödinger equation directly, as shown in the diagonal line of Figure 1, by taking $N \rightarrow \infty$ and $\hbar \rightarrow 0$ simultaneously. In order to do this, it is convenient for us to introduce the second quantization framework in our study of the quantum many-body systems. In particular, we utilize the notations in $[6,8,11]$ where the fermionic Fock space is defined as

$$
\mathcal{F}_{a}=\bigoplus_{n \geq 0} L_{a}^{2}\left(\mathbb{R}^{3 n},(\mathrm{~d} x)^{\otimes n}\right),
$$

where we denote $(\mathrm{d} x)^{\otimes n}=\mathrm{d} x_{1} \cdots \mathrm{d} x_{n}$. The creation and annihilation operator in terms of their respective distributive forms,

$$
a^{*}(f)=\int \mathrm{d} x a_{x}^{*} f(x), \quad a(f)=\int \mathrm{d} x a_{x} \overline{f(x)} .
$$

${ }^{1}$ See Figure 1. 
Due to the canonical anti-commutator relation (CAR) in the fermionic regime, we have that for all $f, g \in H^{1}\left(\mathbb{R}^{3}\right)$

$$
\left\{a(f), a^{*}(g)\right\}=\langle f, g\rangle, \quad\left\{a^{*}(f), a^{*}(g)\right\}=\{a(f), a(g)\}=0,
$$

where $\{A, B\}=A B+B A$ is the anti-commutator. In particular, the CAR for operator kernels hold as follow

$$
\left\{a_{x}, a_{y}^{*}\right\}=\delta_{x=y}, \quad\left\{a_{x}^{*}, a_{y}^{*}\right\}=\left\{a_{x}, a_{y}\right\}=0 .
$$

This CAR in distributive form will be frequently used in our computations.

As in [6], we may write the corresponding Hamiltonian in terms of the operator valued distribution in $\mathcal{F}_{a}$ by

$$
\mathcal{H}_{N}=\frac{\hbar^{2}}{2} \int \mathrm{d} x \nabla_{x} a_{x}^{*} \nabla_{x} a_{x}+\frac{1}{2 N} \iint \mathrm{d} x \mathrm{~d} y V(x-y) a_{x}^{*} a_{y}^{*} a_{y} a_{x} .
$$

Therefore, we rewrite the Schrödinger equation in Fock space as follows,

$$
\left\{\begin{array}{l}
\mathrm{i} \hbar \partial_{t} \psi_{N, t}=\mathcal{H}_{N} \psi_{N, t}, \\
\psi_{N, 0}=\psi_{N},
\end{array}\right.
$$

for all $\psi_{N, t} \in \mathcal{F}_{a}^{(N)}$ and $t \in[0, T]$, where $\psi_{N} \in \mathcal{F}_{a}^{(N)}$ with $\left\|\psi_{N}\right\|=1$. The solution to the above Cauchy problem is $\psi_{N, t}=e^{-\frac{i}{\hbar} \mathcal{H} t} \psi_{N}$, with a given initial data $\psi_{N}$.

Remark 1.1 It should be noted the states $\psi_{N, t}$ in our analysis stays in the $N$ th-sector of $\mathcal{F}_{a}$ due to the definition of Husimi measure which will be given later. Therefore, denoting $\mathcal{F}_{a}^{(n)}$ to be the $n$-th sector in $\mathcal{F}_{a}$, we say that $\psi_{N, t} \in \mathcal{F}_{a}^{(N)}$ for all $t \geq 0$.

Furthermore, we use the definition of the number and kinetic energy operators as follows,

$$
\mathcal{N}=\int \mathrm{d} x a_{x}^{*} a_{x} \text { and } \mathcal{K}=\hbar^{2} \int \mathrm{d} x \nabla_{x} a_{x}^{*} \nabla_{x} a_{x}
$$

respectively. We further explore the properties of the operators in (1.9) in Sect. 2.2.2.

Next, we shall introduce the Husimi measure. In fact, our notation follows closely with the notations in Fournais et al. [19] where it deals with large fermionic particles in stationary case. The main tool in their analysis is the use of coherent state, a subtle tool that proves extremely useful in our work as well.

For any real-valued normalized function $f$, the coherent state is given by, ${ }^{2}$

$$
f_{q, p}^{\hbar}(y):=\hbar^{-\frac{3}{4}} f\left(\frac{y-q}{\sqrt{\hbar}}\right) e^{\frac{i}{\hbar} p \cdot y},
$$

Similar to [12] and [19], the $k$-particle Husimi measure is defined as, for any $1 \leq k \leq N$

$$
m_{N}^{(k)}\left(q_{1}, p_{1}, \ldots, q_{k}, p_{k}\right):=\left\langle\psi_{N}, a^{*}\left(f_{q_{1}, p_{1}}^{\hbar}\right) \cdots a^{*}\left(f_{q_{k}, p_{k}}^{\hbar}\right) a\left(f_{q_{k}, p_{k}}^{\hbar}\right) \cdots a\left(f_{q_{1}, p_{1}}^{\hbar}\right) \psi_{N}\right\rangle,
$$

where $\psi_{N} \in \mathcal{F}_{a}^{(N)}$ is the $N$-fermionic states, $a\left(f_{q, p}^{\hbar}\right)$ and $a^{*}\left(f_{q, p}^{\hbar}\right)$ are the annihilation and creation operators respectively. Husimi measure defined in (1.11) measures how many particles, in particularly fermions, are in the $k$ semiclassical boxes with length scaled of $\sqrt{\hbar}$ centered in its respectively phase-space pair, $\left(q_{1}, p_{1}\right), \ldots,\left(q_{k}, p_{k}\right)$.

2 The function $f$ can be any real-valued function. [19] For this paper, we set $f$ to be compactly supported. See Assumption A1. 
In the context of this paper, we use $m_{N, t}^{(k)}$ to be the time dependent Husimi measure defined by the solution of the Schrödinger equation $\psi_{N, t}$. By using operator kernels defined in (1.4), we may rewrite the Husimi measure as follows

$$
\begin{aligned}
& m_{N, t}^{(k)}\left(q_{1}, p_{1}, \ldots, q_{k}, p_{k}\right) \\
& \quad:=\int \cdots \int(\mathrm{d} w \mathrm{~d} u)^{\otimes k}\left(f_{q, p}^{\hbar}(w) \overline{f_{q, p}^{\hbar}(u)}\right)^{\otimes k}\left\langle\psi_{N, t}, a_{w_{1}}^{*} \cdots a_{w_{k}}^{*} a_{u_{k}} \cdots a_{u_{1}} \psi_{N, t}\right\rangle,
\end{aligned}
$$

where the tensor products indicate

$$
(\mathrm{d} w \mathrm{~d} u)^{\otimes k}:=\mathrm{d} w_{1} \mathrm{~d} u_{1} \cdots \mathrm{d} w_{k} \mathrm{~d} u_{k}
$$

and

$$
\left(f_{q, p}^{\hbar}(w) \overline{f_{q, p}^{\hbar}(u)}\right)^{\otimes k}:=\prod_{j=1}^{k} f_{q_{j}, p_{j}}^{\hbar}\left(w_{j}\right) \overline{f_{q_{j}, p_{j}}^{\hbar}\left(u_{j}\right)} .
$$

Note that the function $f$ here is a very well localized function in practice [19], therefore we may take the following assumption

Assumption A1 The real-valued function $f \in H^{1}\left(\mathbb{R}^{3}\right)$ satisfies $\|f\|_{2}=1$, and has compact support.

Additionally, we assume that the interaction potential to satisfy

Assumption A2 $V$ is a real-valued function such that $V(-x)=V(x)$ and $V \in W^{2, \infty}\left(\mathbb{R}^{3}\right)$.

As is well known that in the mean field semiclassical regime, the dynamic of (1.1) can be approximated by a one particle Vlasov equation. Namely, for all $q, p \in \mathbb{R}^{3}$

$$
\partial_{t} m_{t}(q, p)+p \cdot \nabla_{q} m_{t}(q, p)=\nabla\left(V * \rho_{t}\right)(q) \cdot \nabla_{p} m_{t}(q, p),
$$

with initial data $m_{0}(q, p)$, where $m_{t}(q, p)$ is the time dependent one particle probability density function, and $\rho_{t}(q)=\int m_{t}(q, p) \mathrm{d} p$. Although (1.13) is a non-linear equation, such equation would be more suitable to analyze than the increasingly large systems of Schrödinger equation. The well-posedness of the above Vlasov problem is given by Drobrushin [15] for smooth $V$.

Now, we are ready to state the our main results.

Theorem 1.1 Let Assumptions A1 and A2 hold, $\psi_{N, t}$ be the solution of Schrödinger equation (1.8), $m_{N, t}^{(k)}$ be the Husimi measure defined in (1.12). If $m_{N}^{(1)}$, the 1-particle Husimi measure of the initial data $\psi_{N}$, satisfies

$$
\iint \mathrm{d} q_{1} \mathrm{~d} p_{1}\left(\left|p_{1}\right|^{2}+\left|q_{1}\right|\right) m_{N}^{(1)}\left(q_{1}, p_{1}\right) \leq C .
$$

Then, for all $t \geq 0$, the $k$-particle Husimi measure at time $t, m_{N, t}^{(k)}$ has a weakly convergent subsequence which converges to $m_{t}^{(k)}$ in $L^{1}\left(\mathbb{R}^{6}\right)$, where $m_{t}^{(k)}$ is a weak solution of the following infinite hierarchy in the sense of distribution, i.e. it satisfies for all $k \geq 1$ that

$$
\begin{aligned}
& \partial_{t} m_{t}^{(k)}\left(q_{1}, p_{1}, \ldots, q_{k}, p_{k}\right)+\mathbf{p}_{k} \cdot \nabla_{\mathbf{q}_{k}} m_{t}^{(k)}\left(q_{1}, p_{1}, \ldots, q_{k}, p_{k}\right) \\
& \quad=\frac{1}{(2 \pi)^{3}} \nabla_{\mathbf{p}_{k}} \cdot \iint \mathrm{d} q_{k+1} \mathrm{~d} p_{k+1} \nabla V\left(q_{j}-q_{k+1}\right) m_{t}^{(k+1)}\left(q_{1}, p_{1}, \ldots, q_{k+1}, p_{k+1}\right) .
\end{aligned}
$$


By using [47, Theorem 7.12], we have the following corollary,

Corollary 1.1 Suppose assumptions A1 and A2 hold. Assume further that the initial data of (1.15) can be factorized, i.e. for all $k \geq 1$,

$$
\left\|m_{N}^{(k)}-m_{0}^{\otimes k}\right\|_{L^{1}} \rightarrow 0, \quad \text { as } N \rightarrow \infty .
$$

Then, if the infinite hierarchy (1.15) has a unique solution and $m_{t}$ is the solution to the classical Vlasov equation in (1.13), it holds that

$$
W_{1}\left(m_{N, t}^{(1)}, m_{t}\right) \longrightarrow 0, \quad \text { as } N \rightarrow \infty,
$$

for $t \geq 0$.

Remark 1.2 In the pioneering work by Spohn [46], he considered

$$
\begin{aligned}
& r_{n}^{(N)}\left(\xi_{1}, \eta_{1}, \ldots, \xi_{N}, \eta_{N}, t\right) \\
& \quad=\operatorname{tr}\left[e^{-\mathrm{i} H_{N} t}\left|\Psi_{N}\right\rangle\left\langle\Psi_{N}\right| e^{\mathrm{i} H_{N} t} \prod_{j=1}^{N} \exp \left(\mathrm{i}\left(N^{-1 / 3} \xi_{j} p_{j}+\eta_{j} x_{j}\right)\right)\right]
\end{aligned}
$$

with $p_{j}=-\mathrm{i} \nabla_{j}$ and obtained the following Vlasov hierarchy,

$$
\begin{aligned}
\frac{\partial}{\partial t} r_{n}^{(N)}\left(\xi_{1}, \eta_{1}, \ldots, \xi_{n}, \eta_{n}, t\right) \\
\quad=\sum_{j=1}^{n} \eta_{j} \frac{\partial}{\partial \xi_{j}} r_{n}^{(N)}\left(\xi_{1}, \eta_{1}, \ldots, \xi_{n}, \eta_{n}, t\right) \\
\quad+\sum_{j=1}^{n} \int \hat{V}(\mathrm{~d} k) k \cdot \xi_{j} r_{n+1}^{(N)}\left(\xi_{1}, \eta_{1}, \ldots, \xi_{j}, \eta_{j}+k, \ldots \xi_{n}, \eta_{n}, 0,-k, t\right),
\end{aligned}
$$

which is slightly different from Vlasov hierarchy for Husimi measure given in (1.15), or the version in (2.3) before taking the limit. The benefit of the hierarchy in (2.3) is that one observes directly the mean field and semiclassical structure in the remainder terms. The explicit formulation is helpful in getting estimates for the remainder terms in (2.3). Moreover if one can handle singular potentials (or even the Coulomb potential) for both terms separately, one expects that this new approach can be applied to obtain the limit from many body Schrördinger to Vlasov with singular potentials in the future. Since the mean field limit with singular potential has been studied with convergence rate, for example in [8], then we can utilize similar ideas to handle one of the remainder term which includes the mean field structure. In parallel, we can apply the techniques in semiclassical limit, for example in [43], to get estimates for the other remainder term.

Remark 1.3 Although the results in this article does not yield a convergent rate, the main purpose of this article is to present an alternative approach and framework, namely to rewrite the Schrödinger equation into a BBGKY type of hierarchy, and to derive estimates for the remainder terms that appear in the new hierarchy.

Remark 1.4 In Corollary 1.1, the convergence is stated in terms of 1-Wasserstein distance. For completeness, we give its definition as defined in [47]

$$
W_{1}(\mu, v):=\max _{\pi \in \Pi(\mu, \nu)} \int|x-y| \mathrm{d} \pi(x, y),
$$


where $\mu$ and $v$ are probability measures and $\Pi(\mu, v)$ the set of all probability measures with marginals $\mu$ and $\nu$. The Wasserstein distance, also known as Monge-Kantorovich distance, is a distance on the set of probability measures. In fact, if we interpret the metric in $L^{p}$ space as the distance that measures two densities "vertically", the Wasserstein distance measures the distance between two densities "horizontally" [44].

Remark 1.5 The assumptions for initial data (1.14) and (1.16) can be realized by choosing $\psi_{N}$ to be the Slater-determinant. That is, for all orthonormal basis $\left\{\varphi_{j}\right\}_{j=1}^{\infty}$, the initial data is given as

$$
\psi_{N}\left(q_{1}, \ldots, q_{N}\right)=\frac{1}{\sqrt{N !}} \operatorname{det}\left\{\varphi_{j}\left(q_{i}\right)\right\}_{1 \leq i, j \leq N},
$$

Remark 1.6 Assumptions A1 and A2 are expected to be weakened to the situation that $f \in H^{1}\left(\mathbb{R}^{3}\right),|x| f(x) \in L^{2}\left(\mathbb{R}^{3}\right)$, and $V$ to be Coulomb potential. These will be our future projects.

Remark 1.7 In this context, we have applied the BBGKY hierarchy, the intermediate mean field approximation Hartree Fock system has not been benefited. With Hartree Fock approximation, one can do direct factorization in the equation for $m_{N, t}^{(1)}$. In this direction, we expect to derive the rate of convergence in an appropriate distance between the Husimi measure and the solution of the Vlasov equation.

The arrangement of the paper is the following. In Sect. 2, we give the main strategy of the proof. Followed by the reformulation of Schrödinger equation into a hierarchy of the Husimi measure, a sequence of necessary estimates on number operators, the localized number operators, and the kinetic energy operator are given, which will be contributed to do compactness argument for the Husimi measure. We leave the computation of the hierarchy to Sect. 3.1. Furthermore, the uniform estimates for remainder terms in the hierarchy, which is another main contribution of this article, are provided in Sect. 3.2.

\section{Proof Strategy Through BBGKY Type Hierarchy for Husimi Measure}

We first start from the many particle Schrödinger equation and derive an approximated hierarchy of time dependent Husimi measure by direct computation. Compare to the BBGKY hierarchy of Liouville equation in the classical sense, it has two families of remainder terms, which are determined by the $N$ particle wave function from Schrödinger equation. In order to take a convergent subsequence of the $k$-particle Husimi measure, we derive the uniform estimates for number operator and the kinetic energy. Together with an additional estimate for localized number operator, we can show that the remainder terms are of order $\hbar^{\frac{1}{2}-\delta}$, for arbitrary small $\delta$. Then the desired result will be obtained by the uniqueness of solution to the infinite hierarchy.

\subsection{Reformulation: Hierarchy of Time Dependent Husimi Measure}

In this subsection, we begin by examining the dynamics of $k$-particle Husimi measure by using the $N$-body fermionic Schrödinger. The proofs of the following propositions are provided in Sect. 3.1. 
Proposition 2.1 Suppose $\psi_{N, t} \in \mathcal{F}_{a}^{(N)}$ is anti-symmetric $N$-particle state satisfying the Schrödinger equation in (1.8). Moreover, if $V(-x)=V(x)$ then we have the following equation for $k=1$,

$$
\begin{aligned}
& \partial_{t} m_{N, t}^{(1)}\left(q_{1}, p_{1}\right)+p_{1} \cdot \nabla_{q_{1}} m_{N, t}^{(1)}\left(q_{1}, p_{1}\right) \\
& \quad=\frac{1}{(2 \pi)^{3}} \nabla_{p_{1}} \cdot \iint \mathrm{d} q_{2} \mathrm{~d} p_{2} \nabla V\left(q_{1}-q_{2}\right) m_{N, t}^{(2)}\left(q_{1}, p_{1}, q_{2}, p_{2}\right)+\nabla_{q_{1}} \cdot \mathcal{R}_{1}+\nabla_{p_{1}} \cdot \widetilde{\mathcal{R}}_{1},
\end{aligned}
$$

where the remainder terms $\mathcal{R}_{1}$ and $\widetilde{\mathcal{R}}_{1}$, are given by

$$
\begin{aligned}
\mathcal{R}_{1}:= & \hbar \operatorname{Im}\left\langle\nabla_{q_{1}} a\left(f_{q_{1}, p_{1}}^{\hbar}\right) \psi_{N, t}, a\left(f_{q_{1}, p_{1}}^{\hbar}\right) \psi_{N, t}\right\rangle, \\
\widetilde{\mathcal{R}}_{1}:= & \frac{1}{(2 \pi)^{3}} \cdot \operatorname{Re} \iint \mathrm{d} w \mathrm{~d} u \iint \mathrm{d} y \mathrm{~d} v \iint \mathrm{d} q_{2} \mathrm{~d} p_{2} \int_{0}^{1} \mathrm{~d} s \\
& \nabla V(s u+(1-s) w-y) f_{q_{1}, p_{1}}^{\hbar}(w) \overline{f_{q_{1}, p_{1}}^{\hbar}(u)} f_{q_{2}, p_{2}}^{\hbar}(y) \overline{f_{q_{2}, p_{2}}^{\hbar}(v)}\left\langle a_{y} a_{w} \psi_{N, t}, a_{v} a_{u} \psi_{N, t}\right\rangle \\
& \quad-\frac{1}{(2 \pi)^{3}} \iint \mathrm{d} q_{2} \mathrm{~d} p_{2} \nabla V\left(q_{1}-q_{2}\right) m_{N, t}^{(2)}\left(q_{1}, p_{1}, q_{2}, p_{2}\right),
\end{aligned}
$$

Proposition 2.2 For every $1 \leq i, j \leq k$ and $q_{j}, p_{j} \in \mathbb{R}^{3}$, denote $\mathbf{q}_{k}=\left(q_{1}, \ldots, q_{k}\right)$ and $\mathbf{p}_{k}=\left(p_{1}, \ldots, p_{k}\right)$. Under the assumption in Proposition 2.1, then for $1<k \leq N$, we have the following hierarchy

$$
\begin{aligned}
& \partial_{t} m_{N, t}^{(k)}\left(q_{1}, p_{1}, \ldots, q_{k}, p_{k}\right)+\mathbf{p}_{k} \cdot \nabla_{\mathbf{q}_{k}} m_{N, t}^{(k)}\left(q_{1}, p_{1}, \ldots, q_{k}, p_{k}\right) \\
& =\frac{1}{(2 \pi)^{3}} \nabla_{\mathbf{p}_{k}} \cdot \iint \mathrm{d} q_{k+1} \mathrm{~d} p_{k+1} \nabla V\left(q_{j}-q_{k+1}\right) m_{N, t}^{(k+1)}\left(q_{1}, p_{1}, \ldots, q_{k+1}, p_{k+1}\right) \\
& \quad+\nabla_{\mathbf{q}_{k}} \cdot \mathcal{R}_{k}+\nabla_{\mathbf{p}_{k}} \cdot \widetilde{\mathcal{R}}_{k}+\widehat{\mathcal{R}}_{k},
\end{aligned}
$$

where the remainder terms are denoted as

$$
\begin{aligned}
\mathcal{R}_{k}:= & \hbar \operatorname{Im}\left\langle\nabla_{\mathbf{q}_{k}}\left(a\left(f_{q_{k}, p_{k}}^{\hbar}\right) \cdots a\left(f_{q_{1}, p_{1}}^{\hbar}\right)\right) \psi_{N, t}, a\left(f_{q_{k}, p_{k}}^{\hbar}\right) \cdots a\left(f_{q_{1}, p_{1}}^{\hbar}\right) \psi_{N, t}\right\rangle, \\
\left(\widetilde{\mathcal{R}}_{k}\right)_{j}:= & \frac{1}{(2 \pi)^{3}} \operatorname{Re} \int \cdots \int(\mathrm{d} w \mathrm{~d} u)^{\otimes k} \int \mathrm{d} y\left[\int_{0}^{1} \mathrm{~d} s \nabla V\left(s u_{j}+(1-s) w_{j}-y\right)\right]\left(f_{q, p}^{\hbar}(w) \overline{f_{q, p}^{\hbar}(u)}\right)^{\otimes k} \\
& \iint \mathrm{d} \widetilde{q} d \widetilde{p} f_{\tilde{q}, \widetilde{p}}^{\hbar}(y) \int \mathrm{d} v \overline{f_{\widetilde{q}, \widetilde{p}}^{\hbar}(v)}\left\langle a_{w_{k}} \cdots a_{w_{1}} a_{y} \psi_{N, t}, a_{u_{k}} \cdots a_{u_{1}} a_{v} \psi_{N, t}\right\rangle \\
& -\frac{1}{(2 \pi)^{3}} \iint \mathrm{d} q_{k+1} \mathrm{~d} p_{k+1} \nabla V\left(q_{j}-q_{k+1}\right) m_{N, t}^{(k+1)}\left(q_{1}, p_{1}, \ldots, q_{k+1}, p_{k+1}\right), \\
\widehat{\mathcal{R}}_{k}:= & \left.\frac{\hbar^{2}}{2} \operatorname{Im} \int \cdots \int(\mathrm{d} w \mathrm{~d} u)^{\otimes k} \sum_{j \neq i}^{k}\left[V\left(u_{j}-u_{i}\right)-V\left(w_{j}-w_{i}\right)\right]\left(f_{q, p}^{\hbar}(w) \overline{f_{q, p}^{\hbar}(u)}\right)\right)^{\otimes k} \\
& \left\langle a_{w_{k}} \cdots a_{w_{1}} \psi_{N, t}, a_{u_{k}} \cdots a_{u_{1}} \psi_{N, t}\right\rangle
\end{aligned}
$$

\subsection{A Priori Estimates}

In the next steps, we derive estimates in order to have compactness of each $k$-particle Husimi measure, as well as to prove that the remainder terms converge to zero in the sense of distri- 
bution. The estimates are derived directly from the solutions of the $N$-fermionic Schrödinger equation.

\subsubsection{Properties of Coherent States and Husimi Measure}

Here we give the properties of coherent states and Husimi measure provided in [19], which will be frequently needed in our computation. Firstly, we observe that the coherent state has a projection property, that is

Lemma 2.1 (Projection of the coherent state, [19]) For every real-valued function $f$ satisfying $\|f\|_{2}=1$ and the coherent states $f_{q, p}^{\hbar}$ defined as in (1.10), we have that

$$
\frac{1}{(2 \pi \hbar)^{3}} \iint \mathrm{d} q \mathrm{~d} p\left|f_{q, p}^{\hbar}\right\rangle\left\langle f_{q, p}^{\hbar}\right|=\frac{1}{(2 \pi \hbar)^{3}} \iint \mathrm{d} q \mathrm{~d} p\left\langle f_{q, p}^{\hbar}, \cdot\right\rangle f_{q, p}^{\hbar}(y)=\mathbb{1} .
$$

Secondly, the properties of the $k$-particle Husimi measure $m_{N}^{(k)}$ is given as follows

Lemma 2.2 (Properties of $k$-particle Husimi measure, [19]) Suppose for $\psi_{N} \in \mathcal{F}_{a}^{(N)}$ is normalized. Then, the following properties hold true for $m_{N}^{(k)}$ :

1. $m_{N}^{(k)}\left(q_{1}, p_{1}, \ldots, q_{k}, p_{k}\right)$ is symmetric,

2. $\frac{1}{(2 \pi)^{3 k}} \int \cdots \int(\mathrm{d} q \mathrm{~d} p)^{\otimes k} m_{N}^{(k)}\left(q_{1}, p_{1}, \ldots, q_{k}, p_{k}\right)=\frac{N(N-1) \cdots(N-k+1)}{N^{k}}$,

3. $\frac{1}{(2 \pi \hbar)^{3}} \iint \mathrm{d} q_{k} \mathrm{~d} p_{k} m_{N}^{(k)}\left(q_{1}, p_{1}, \ldots, q_{k}, p_{k}\right)=(N-k+1) m_{N}^{(k-1)}\left(q_{1}, p_{1}, \ldots, q_{k-1}, p_{k-1}\right)$, and

4. $0 \leq m_{N}^{(k)}\left(q_{1}, p_{1}, \ldots, q_{k}, p_{k}\right) \leq 1$ a.e.,

where $1 \leq k \leq N$.

Remark 2.1 Note that as $\left\|\psi_{N}\right\|=\left\|\psi_{N, t}\right\|$, Lemma 2.2 is also valid if we replaced the stationary wave-function $\psi_{N}$, to a time-dependent $\psi_{N, t}$, for $t \geq 0$. Moreover, it can be obtained that for any fixed positive integer $1 \leq k \leq N$,

$$
0 \leq m_{N, t}^{(k)} \leq 1 \quad \text { a.e. in } \mathbb{R}^{6 k} \text {. }
$$

Following [19], we define the $\hbar$-weighted Fourier transformation as follows,

Definition 2.1 ( $\hbar$-weighted Fourier transform) Let $F$ be any real-valued function in $L^{2}\left(\mathbb{R}^{3}\right)$. We define the $\hbar$-weighted Fourier transform of $f$ to be,

$$
\mathcal{F}_{\hbar}[f](p):=\frac{1}{(2 \pi \hbar)^{\frac{3}{2}}} \int_{\mathbb{R}^{3}} \mathrm{~d} x f(x) e^{-\frac{\mathrm{i}}{\hbar} p \cdot x},
$$

and its inverse transform by $\mathcal{F}_{\hbar}^{-1}$.

From the Definition 2.1, we have the following identity,

$$
\int_{\mathbb{R}^{3}} \mathrm{~d} y G(y) F(y)=\int_{\mathbb{R}^{3}} \mathrm{~d} y G(y) \frac{1}{(2 \pi \hbar)^{3}} \iint_{\mathbb{R}^{3 \cdot 2}} \mathrm{~d} p_{2} \mathrm{~d} v F(v) e^{\frac{\mathrm{i}}{\hbar} p_{2} \cdot(y-v)},
$$

for any $G, F \in L^{2}\left(\mathbb{R}^{3}\right)$. In other words, the Dirac-delta distribution is given by

$$
\delta_{y}(v)=\frac{1}{(2 \pi \hbar)^{3}} \int_{\mathbb{R}^{3}} \mathrm{~d} p_{2} e^{\frac{\mathrm{i}}{\hbar} p_{2} \cdot(y-v)} .
$$




\subsubsection{Number Operator and Localized Number Operator}

In this part, we give the bounds of number operators and its corresponding localized version, both of which are used extensively in estimating the remainder terms in (2.1) and (2.3).

Lemma 2.3 Let $\psi_{N, t} \in \mathcal{F}_{a}^{(N)}$ be the solution to Schrödinger equation in (1.1) with initial data $\left\|\psi_{N}\right\|=1$, the number operator $\mathcal{N}$ defined in (1.9). Then, for finite $1 \leq k \leq N$, we have

$$
\left\langle\psi_{N, t}, \frac{\mathcal{N}^{k}}{N^{k}} \psi_{N, t}\right\rangle=1 .
$$

Proof Since $\psi_{N, t}$ satisfies the Schrödinger equation, then for $k \geq 1$,

$$
i \hbar \frac{\mathrm{d}}{\mathrm{d} t}\left\langle\psi_{N, t}, \mathcal{N}^{k} \psi_{N, t}\right\rangle=\left\langle\psi_{N, t},\left[\mathcal{N}^{k}, \mathcal{H}_{N}\right] \psi_{N, t}\right\rangle=k\left\langle\psi_{N, t}, \mathcal{N}^{k-1}\left[\mathcal{N}, \mathcal{H}_{N}\right] \psi_{N, t}\right\rangle=0,
$$

where we used the fact that $\mathcal{H}_{N}$ is self-adjoint and $\left[\mathcal{H}_{N}, \mathcal{N}\right]=0$. Therefore, integrating the above equation with respect to time, gives us

$$
\left\langle\psi_{N, t}, \frac{\mathcal{N}^{k}}{N^{k}} \psi_{N, t}\right\rangle=\left\langle\psi_{N}, \frac{\mathcal{N}^{k}}{N^{k}} \psi_{N}\right\rangle=1,
$$

for any $1 \leq k \leq N$.

Remark 2.2 The expectation of the number operator is the total mass of Husimi measure. In fact, observe that

$$
\left\langle\psi_{N, t}, \mathcal{N} \psi_{N, t}\right\rangle=\int \mathrm{d} x\left\langle\psi_{N, t}, a_{x}^{*} a_{x} \psi_{N, t}\right\rangle=\int \mathrm{d} x\left\langle\psi_{N, t}, a_{x}^{*} 1 a_{x} \psi_{N, t}\right\rangle,
$$

Then, by (2.5)

$$
\begin{aligned}
& =\frac{1}{(2 \pi \hbar)^{3}} \iint \mathrm{d} q \mathrm{~d} p \int \mathrm{d} x\left\langle\psi_{N, t}, a_{x}^{*} f_{q, p}^{\hbar}(x)\left(\int \mathrm{d} y a_{y} \overline{f_{q, p}^{\hbar}(y)}\right) \psi_{N, t}\right\rangle \\
& =\frac{1}{(2 \pi \hbar)^{3}} \iint \mathrm{d} q \mathrm{~d} p\left\langle\psi_{N, t}, a^{*}\left(f_{q, p}^{\hbar}\right) a\left(f_{q, p}^{\hbar}\right) \psi_{N, t}\right\rangle \\
& =\frac{1}{(2 \pi \hbar)^{3}} \iint \mathrm{d} q \mathrm{~d} p m_{N, t}^{(1)}(q, p) \\
& =N
\end{aligned}
$$

where we use Lemma 2.2 in the last equality. Moverover, if we repeat the projection above for $k$-times, we get

$$
\begin{aligned}
& \frac{1}{(2 \pi)^{3 k}} \int \ldots \int(\mathrm{d} q \mathrm{~d} p)^{\otimes k} m_{N, t}^{(k)}\left(q_{1}, p_{1}, \ldots, q_{k}, p_{k}\right) \\
& \leq\left\langle\psi_{N, t}, \frac{\mathcal{N}^{k}}{N^{k}} \psi_{N, t}\right\rangle=1,
\end{aligned}
$$

where $1 \leq k \leq N$ and $t \geq 0$.

More importantly, we have the following estimates for localized number operators. 
Lemma 2.4 (Bound on localized number operator) Let $\psi_{N} \in \mathcal{F}_{a}^{(N)}$ such that $\left\|\psi_{N}\right\|=1$, and $R$ be the radius of a ball such that the volume is 1 . Then, for all $1 \leq k \leq N$, we have

$$
\int \cdots \int(\mathrm{d} q \mathrm{~d} x)^{\otimes k}\left\langle\psi_{N},\left(\prod_{n=1}^{k} \chi_{\left|x_{n}-q_{n}\right| \leq \sqrt{\hbar} R}\right) a_{x_{1}}^{*} \cdots a_{x_{k}}^{*} a_{x_{k}} \cdots a_{x_{1}} \psi_{N}\right\rangle \leq \hbar^{-\frac{3}{2} k},
$$

where $\chi$ is a characteristic function

Proof Consider first the case where $k=1$. For every $1 \leq j \leq k$, we have

$$
\begin{aligned}
& \int \mathrm{d} x_{j}\left(\int \mathrm{d} q_{j} \chi_{\left|x_{j}-q_{j}\right| \leq \sqrt{\hbar} R}\right)\left\langle\psi_{N}, a_{x_{j}}^{*} a_{x_{j}} \psi_{N}\right\rangle \\
& =\hbar^{\frac{3}{2}}\left\langle\psi_{N}, \mathcal{N} \psi_{N}\right\rangle=\hbar^{\frac{3}{2}-3}\left\langle\psi_{N}, \frac{\mathcal{N}}{N} \psi_{N}\right\rangle \leq \hbar^{-\frac{3}{2}},
\end{aligned}
$$

where we used Lemma 2.3. Analogously, for $2 \leq k \leq N$,

$$
\begin{aligned}
& \int(\mathrm{d} x)^{\otimes k}\left(\prod_{n=1}^{k} \int \mathrm{d} q_{n} \chi_{\left|x_{n}-q_{n}\right| \leq \sqrt{\hbar} R}\right)\left\langle\psi_{N}, a_{x_{1}}^{*} \cdots a_{x_{k}}^{*} a_{x_{k}} \cdots a_{x_{1}} \psi_{N}\right\rangle \\
& \leq \hbar^{\frac{3}{2} k}\left\langle\psi_{N}, \mathcal{N}^{k} \psi_{N}\right\rangle=\hbar^{\frac{3}{2} k-3 k}\left\langle\psi_{N}, \frac{\mathcal{N}^{k}}{N^{k}} \psi_{N}\right\rangle \leq \hbar^{\frac{3}{2} k-3 k},
\end{aligned}
$$

where we applied Lemma 2.3 again.

Lemma 2.5 (Estimate of oscillation) For $\varphi(p) \in C_{0}^{\infty}\left(\mathbb{R}^{3}\right)$ and

$$
\Omega_{\hbar}:=\left\{x \in \mathbb{R}^{3} ; \max _{1 \leq j \leq 3}\left|x_{j}\right| \leq \hbar^{\alpha}\right\}
$$

it holds for every $\alpha \in(0,1), s \in \mathbb{N}$, and $x \in \mathbb{R}^{3} \backslash \Omega_{\hbar}$,

$$
\left|\int_{\mathbb{R}^{3}} \mathrm{~d} p e^{\frac{\mathrm{i}}{\hbar} p \cdot x} \varphi(p)\right| \leq C \hbar^{(1-\alpha) s},
$$

where $C$ depends on the compact support and the $C^{s}$ norm of $\varphi$.

Proof We will prove the lemma in a single-variable environment. That is, we let the momentum and space to be $p=\left(p_{1}, p_{2}, p_{3}\right)$ and $x=\left(x_{1}, x_{2}, x_{3}\right)$ such that $x_{j}, p_{j} \in \mathbb{R}$ for all $j \in\{1,2,3\}$. Then, for arbitrary $x \in \mathbb{R}^{3} \backslash \Omega_{\hbar}$, one of the $x_{j}$ s is bigger than $\hbar^{\alpha}$. Without loss of generality, we assume that $\left|x_{1}\right|>\hbar^{\alpha}$ and $x_{2}, x_{3} \in \mathbb{R}$. Let supp $\varphi \subset B_{r}(0) \subset \mathbb{R}^{3}$, we can rewrite the left hand of (2.11) into the following,

$$
\begin{aligned}
& \left|\int_{-r}^{r} \mathrm{~d} p_{1} \int_{-r}^{r} \mathrm{~d} p_{2} \int_{-r}^{r} \mathrm{~d} p_{3} e^{\frac{\mathrm{i}}{\hbar}\left(p_{1} x_{1}+p_{2} x_{2}+p_{3} x_{3}\right)} \varphi(p)\right| \\
& \quad=\left|\int_{-r}^{r} \mathrm{~d} p_{2} e^{\frac{\mathrm{i}}{\hbar} p_{2} x_{2}} \int_{-r}^{r} \mathrm{~d} p_{3} e^{\frac{\mathrm{i}}{\hbar} p_{3} x_{3}} \int_{-r}^{r} \mathrm{~d} p_{1} e^{\frac{\mathrm{i}}{\hbar} p_{1} x_{1}} \varphi(p)\right|
\end{aligned}
$$

Observe that since

$$
-\mathrm{i} \frac{\hbar}{x_{1}} \frac{\mathrm{d}}{\mathrm{d} p_{1}} e^{\frac{\mathrm{i}}{\hbar} p_{1} x_{1}}=e^{\frac{\mathrm{i}}{\hbar} p_{1} x_{1}}
$$


we have after $s$ times integration by parts in $p_{1}$,

$$
\begin{aligned}
& \left|\int_{-r}^{r} \mathrm{~d} p_{1} \int_{-r}^{r} \mathrm{~d} p_{2} \int_{-r}^{r} \mathrm{~d} p_{3} e^{\frac{\mathrm{i}}{\hbar}\left(p_{1} x_{1}+p_{2} x_{2}+p_{3} x_{3}\right)} \varphi(p)\right| \\
& \quad=\left|\left(-\mathrm{i} \frac{\hbar}{x_{1}}\right)^{s} \int_{-r}^{r} \mathrm{~d} p_{2} e^{\frac{\mathrm{i}}{\hbar} p_{2} x_{2}} \int_{-r}^{r} \mathrm{~d} p_{3} e^{\frac{\mathrm{i}}{\hbar} p_{3} x_{3}} \int_{-r}^{r} \mathrm{~d} p_{1} e^{\frac{\mathrm{i}}{\hbar} p_{1} x_{1}} \partial_{p_{1}}^{s} \varphi(p)\right| \\
& \quad \leq C \frac{\hbar^{s}}{\left|x_{1}\right|^{s}} \leq C \hbar^{(1-\alpha) s},
\end{aligned}
$$

where $s$ indicates the number of time that integration by parts has been performed.

\subsubsection{Finite Moments of Husimi Measure}

To prove that the second moment in $p$ of the Husimi measure is finite, we first show that the kinetic energy is bounded from above. Recall that the definition of the kinetic energy operator $\mathcal{K}$, i.e.,

$$
\mathcal{K}=\frac{\hbar^{2}}{2} \int \mathrm{d} x \nabla_{x} a_{x}^{*} \nabla_{x} a_{x},
$$

and the kinetic energy associated with $\psi_{N}$ is given as $\left\langle\psi_{N}, \mathcal{K} \psi_{N}\right\rangle$.

Lemma 2.6 Assume $V \in W^{1, \infty}$, then the kinetic energy is bounded in the following

$$
\left\langle\psi_{N, t}, \frac{\mathcal{K}}{N} \psi_{N, t}\right\rangle \leq 2\left\langle\psi_{N}, \frac{\mathcal{K}}{N} \psi_{N}\right\rangle+C t^{2}
$$

where $C$ depends on $\|\nabla V\|_{\infty}$.

Proof From the Schrödinger equation, we get

$$
i \hbar \frac{\mathrm{d}}{\mathrm{d} t}\left\langle\psi_{N, t}, \mathcal{K} \psi_{N, t}\right\rangle=\left\langle\psi_{N, t},[\mathcal{K}, \mathcal{H}] \psi_{N, t}\right\rangle .
$$

Note that since the commutator between kinetic and interaction term is given as

$$
\begin{aligned}
{[\mathcal{K}, \mathcal{H}] } & =\frac{\hbar^{2}}{4}\left[\int \mathrm{d} x \nabla_{x} a_{x}^{*} \nabla_{x} a_{x}, \iint \mathrm{d} y \mathrm{~d} z V(y-z) a_{y}^{*} a_{z}^{*} a_{z} a_{y}\right] \\
& =\frac{\hbar^{2}}{4} \iint \mathrm{d} x \mathrm{~d} y \nabla_{x} V(x-y)\left(\nabla_{x} a_{x}^{*} a_{y}^{*} a_{y} a_{x}-a_{x}^{*} a_{y}^{*} a_{y} \nabla_{x} a_{x}\right) \\
& =\frac{\hbar^{2}}{2 N} \operatorname{Im} \iint \mathrm{d} x \mathrm{~d} y \nabla_{x} V(x-y)\left(\nabla_{x} a_{x}^{*} a_{y}^{*} a_{y} a_{x}\right)
\end{aligned}
$$

Then, from (2.13), we have that

$$
\frac{1}{N} \frac{\mathrm{d}}{\mathrm{d} t}\left\langle\psi_{N, t}, \mathcal{K} \psi_{N, t}\right\rangle=\frac{\hbar}{2 N^{2}} \operatorname{Im} \iint \mathrm{d} x \mathrm{~d} y \nabla_{x} V(x-y)\left\langle\psi_{N, t}, \nabla_{x} a_{x}^{*} a_{y}^{*} a_{y} a_{x} \psi_{N, t}\right\rangle .
$$

Now, observe that

$$
\begin{aligned}
& \left|\frac{\hbar}{2 N^{2}} \iint \mathrm{d} x \mathrm{~d} y \nabla_{x} V(x-y)\left\langle\psi_{N, t}, \nabla_{x} a_{x}^{*} a_{y}^{*} a_{y} a_{x} \psi_{N, t}\right\rangle\right| \\
& \quad \leq \frac{\hbar}{2 N^{2}}\|\nabla V\|_{L^{\infty}} \iint \mathrm{d} x \mathrm{~d} y\left\|a_{y} \nabla_{x} a_{x} \psi_{N, t}\right\|\left\|a_{y} a_{x} \psi_{N, t}\right\|
\end{aligned}
$$




$$
\begin{aligned}
& \leq C \frac{\hbar}{2 N^{2}}\left(\iint \mathrm{d} x \mathrm{~d} y\left\langle\psi_{N, t}, \nabla_{x} a_{x}^{*} a_{y}^{*} a_{y} \nabla_{x} a_{x} \psi_{N, t}\right\rangle\right)^{\frac{1}{2}}\left(\iint \mathrm{d} x \mathrm{~d} y\left\langle\psi_{N, t}, a_{x}^{*} a_{y}^{*} a_{y} a_{x} \psi_{N, t}\right\rangle\right)^{\frac{1}{2}} \\
& =C\left(\frac{\hbar^{2}}{N} \int \mathrm{d} x\left\langle\psi_{N, t}, \nabla_{x} a_{x}^{*} \frac{\mathcal{N}}{N} \nabla_{x} a_{x} \psi_{N, t}\right\rangle\right)^{\frac{1}{2}}\left\langle\psi_{N, t}, \frac{\mathcal{N}^{2}}{N^{2}} \psi_{N, t}\right\rangle^{\frac{1}{2}} \\
& \leq C\left(\left\langle\psi_{N, t}, \frac{\mathcal{K}}{N} \psi_{N, t}\right\rangle\right)^{\frac{1}{2}},
\end{aligned}
$$

Thus, we have

$$
\frac{\mathrm{d}}{\mathrm{d} t}\left\langle\psi_{N, t}, \frac{\mathcal{K}}{N} \psi_{N, t}\right\rangle \leq C\left\langle\psi_{N, t}, \frac{\mathcal{K}}{N} \psi_{N, t}\right\rangle^{\frac{1}{2}} .
$$

Integrating both sides with respect to time $t$ and we obtain the desired inequality.

Proposition 2.3 For $t \geq 0$, assume Al and let $m_{N, t}^{(k)}$ to be the $k$-particle Husimi measure. Denoting the phase-space vectors $\mathbf{q}_{k}=\left(q_{1}, \ldots, q_{k}\right)$ and $\mathbf{p}_{k}=\left(p_{1}, \ldots, p_{k}\right)$, we have the following finite moments,

$$
\int \ldots \int(\mathrm{d} q \mathrm{~d} p)^{\otimes k}\left(\left|\mathbf{q}_{k}\right|+\left|\mathbf{p}_{k}\right|^{2}\right) m_{N, t}^{(k)}\left(q_{1}, \ldots, p_{k}\right) \leq C\left(1+t^{3}\right)
$$

where $C$ is a constant dependent on $k, \iint \mathrm{d} q_{1} \mathrm{~d} p_{1}\left(\left|q_{1}\right|+\left|p_{1}\right|^{2}\right) m_{N}^{(1)}\left(q_{1}, p_{1}\right)$, and $\|\nabla V\|_{\infty}$.

Proof We first consider the case where $k=1$. Observe that we may rewrite the kinetic energy as follows

$$
\begin{aligned}
& \frac{1}{N}\left\langle\psi_{N, t}, \mathcal{K} \psi_{N, t}\right\rangle=\frac{\hbar^{2}}{N} \int \mathrm{d} w\left\langle\psi_{N, t}, \nabla_{w} a_{w}^{*} \nabla_{w} a_{w} \psi_{N, t}\right\rangle \\
& =\frac{\hbar^{2}}{N}(2 \pi \hbar)^{-3} \iint \mathrm{d} q_{1} \mathrm{~d} p_{1} \iint \mathrm{d} w \mathrm{~d} u f_{q_{1}, p_{1}}^{\hbar}(w) \overline{f_{q_{1}, p_{1}}^{\hbar}(u)}\left\langle\psi_{N, t}, \nabla_{w} a_{w}^{*} \nabla_{u} a_{u} \psi_{N, t}\right\rangle \\
& =\frac{\hbar^{2}}{(2 \pi)^{3}} \iint \mathrm{d} q_{1} \mathrm{~d} p_{1} \iint \mathrm{d} w \mathrm{~d} u \nabla_{w} f_{q_{1}, p_{1}}^{\hbar}(w) \overline{\nabla_{u} f_{q_{1}, p_{1}}^{\hbar}(u)}\left\langle\psi_{N, t}, a_{w}^{*} a_{u} \psi_{N, t}\right\rangle \\
& =\frac{\hbar^{2}}{(2 \pi)^{3}} \iint \mathrm{d} q_{1} \mathrm{~d} p_{1} \iint \mathrm{d} w \mathrm{~d} u\left(-\nabla_{q_{1}}+i \hbar^{-1} p_{1}\right) f_{q_{1}, p_{1}}^{\hbar}(w) \\
& \quad \cdot\left(-\nabla_{q_{1}}-i \hbar^{-1} p_{1}\right) \overline{f_{q_{1}, p_{1}}^{\hbar}(u)}\left\langle\psi_{N, t}, a_{w}^{*} a_{u} \psi_{N, t}\right\rangle,
\end{aligned}
$$

where we used the fact that

$$
\nabla_{w} f\left(\frac{w-q_{1}}{\sqrt{\hbar}}\right)=-\nabla_{q_{1}} f\left(\frac{w-q_{1}}{\sqrt{\hbar}}\right) .
$$

To continue, we have

$$
\begin{aligned}
\frac{1}{N}\left\langle\psi_{N, t}, \mathcal{K} \psi_{N, t}\right\rangle= & \frac{1}{(2 \pi)^{3}} \iint \mathrm{d} q_{1} \mathrm{~d} p_{1}\left|p_{1}\right|^{2} m_{N, t}^{(1)}\left(q_{1}, p_{1}\right) \\
& +\frac{\hbar^{2}}{(2 \pi)^{3}} \iint \mathrm{d} q_{1} \mathrm{~d} p_{1} \iint \mathrm{d} w \mathrm{~d} u \nabla_{q_{1}} f_{q_{1}, p_{1}}^{\hbar}(w) \cdot \nabla_{q_{1}} \overline{f_{q_{1}, p_{1}}^{\hbar}(u)}\left\langle\psi_{N, t}, a_{w}^{*} a_{u} \psi_{N, t}\right\rangle \\
& +\hbar \frac{2 i}{(2 \pi)^{3}} \operatorname{Im} \iint \mathrm{d} q_{1} \mathrm{~d} p_{1} \iint \mathrm{d} w \mathrm{~d} u p_{1} \cdot \nabla_{q_{1}} f_{q_{1}, p_{1}}^{\hbar}(w) \overline{f_{q_{1}, p_{1}}^{\hbar}(u)}\left\langle\psi_{N, t}, a_{w}^{*} a_{u} \psi_{N, t}\right\rangle .
\end{aligned}
$$


Since kinetic energy is real-valued, if we take the real part of (2.14), the last term in the right hand side vanishes since it is purely imaginary, yielding

$$
\begin{aligned}
\frac{1}{N}\left\langle\psi_{N, t}, \mathcal{K} \psi_{N, t}\right\rangle= & \frac{1}{(2 \pi)^{3}} \iint \mathrm{d} q_{1} \mathrm{~d} p_{1}\left|p_{1}\right|^{2} m_{N, t}^{(1)}\left(q_{1}, p_{1}\right) \\
& +\frac{\hbar^{2}}{(2 \pi)^{3}} \operatorname{Re} \iint \mathrm{d} q_{1} \mathrm{~d} p_{1} \iint \mathrm{d} w \mathrm{~d} u \nabla_{q_{1}} f_{q_{1}, p_{1}}^{\hbar}(w) \cdot \nabla_{q_{1}} \overline{f_{q_{1}, p_{1}}^{\hbar}(u)}\left\langle\psi_{N, t}, a_{w}^{*} a_{u} \psi_{N, t}\right\rangle .
\end{aligned}
$$

Note that by (2.7), we have

$$
\begin{aligned}
& \frac{\hbar^{2}}{(2 \pi)^{3}} \iint \mathrm{d} q_{1} \mathrm{~d} p_{1} \iint \mathrm{d} w \mathrm{~d} u \nabla_{q_{1}} f_{q_{1}, p_{1}}^{\hbar}(w) \cdot \nabla_{q_{1}} \overline{f_{q_{1}, p_{1}}^{\hbar}(u)}\left\langle\psi_{N, t}, a_{w}^{*} a_{u} \psi_{N, t}\right\rangle \\
& =\hbar^{2+3} \iint \mathrm{d} q_{1} \mathrm{~d} w \hbar^{-\frac{3}{2}}\left|\nabla_{q_{1}} f\left(\frac{w-q_{1}}{\sqrt{\hbar}}\right)\right|^{2}\left\langle\psi_{N, t}, a_{w}^{*} a_{w} \psi_{N, t}\right\rangle \\
& =\hbar \int \mathrm{d} \widetilde{q}|\nabla f(\widetilde{q})|^{2}\left\langle\psi_{N, t}, \frac{\mathcal{N}}{N} \psi_{N, t}\right\rangle \\
& =\hbar \int \mathrm{d} \widetilde{q}|\nabla f(\widetilde{q})|^{2},
\end{aligned}
$$

where we recall that $\hbar^{3}=N^{-1}$. Thus, taking the real part of (2.14), we have that

$$
\left\langle\psi_{N, t}, \frac{\mathcal{K}}{N} \psi_{N, t}\right\rangle=\frac{1}{(2 \pi)^{3}} \iint \mathrm{d} q_{1} \mathrm{~d} p_{1}\left|p_{1}\right|^{2} m_{N, t}^{(1)}\left(q_{1}, p_{1}\right)+\hbar \int \mathrm{d} q|\nabla f(q)|^{2},
$$

which means,

$$
\frac{1}{(2 \pi)^{3}} \iint \mathrm{d} q_{1} \mathrm{~d} p_{1}\left|p_{1}\right|^{2} m_{N, t}^{(1)}\left(q_{1}, p_{1}\right) \leq\left\langle\psi_{N, t}, \frac{\mathcal{K}}{N} \psi_{N, t}\right\rangle .
$$

Therefore, (2.17) tells us that the second moment of the 1-particle Husimi measure in momentum space is finite if the kinetic energy is finite.

Now, we turn our focus on the moment with respect to position space. From (2.1), we get

$\partial_{t} \iint \mathrm{d} q_{1} \mathrm{~d} p_{1}\left|q_{1}\right| m_{N, t}^{(1)}\left(q_{1}, p_{1}\right)=\iint\left|q_{1}\right| \partial_{t} m_{N, t}^{(1)}\left(q_{1}, p_{1}\right)$

$$
\begin{aligned}
& =\iint \mathrm{d} q_{1} \mathrm{~d} p_{1}\left|q_{1}\right|\left(-p_{1} \cdot \nabla_{q_{1}} m_{N, t}^{(1)}\left(q_{1}, p_{1}\right)+\frac{1}{(2 \pi)^{3}} \nabla_{p_{1}} \cdot \iint \mathrm{d} w \mathrm{~d} u \iint \mathrm{d} x \mathrm{~d} y \iint \mathrm{d} q_{2} \mathrm{~d} p_{2} \int_{0}^{1} \mathrm{~d} s\right. \\
& \left.\nabla V(s u+(1-s) w-x) f_{q_{1}, p_{1}}^{\hbar}(w) \overline{f_{q_{1}, p_{1}}^{\hbar}(u)} f_{q_{2}, p_{2}}^{\hbar}(x) \overline{f_{q_{2}, p_{2}}^{\hbar}(y)}\left\langle a_{x} a_{w} \psi_{N, t}, a_{y} a_{u} \psi_{N, t}\right\rangle+\nabla_{q_{1}} \cdot \mathcal{R}_{1}\right) .
\end{aligned}
$$

Then, using intergration by parts with respect to $p_{1}$,

$$
\begin{aligned}
& =\iint \mathrm{d} q_{1} \mathrm{~d} p_{1} \nabla_{q_{1}}\left|q_{1}\right| \cdot\left(p_{1} m_{N, t}^{(1)}\left(q_{1}, p_{1}\right)+\mathcal{R}_{1}\right) \\
& =\iint \mathrm{d} q_{1} \mathrm{~d} p_{1} \frac{q_{1}}{\left|q_{1}\right|} \cdot\left(p_{1} m_{N, t}^{(1)}\left(q_{1}, p_{1}\right)+\mathcal{R}_{1}\right) \\
& \leq \iint \mathrm{d} q_{1} \mathrm{~d} p_{1}\left(\left|p_{1}\right| m_{N, t}^{(1)}\left(q_{1}, p_{1}\right)+\left|\mathcal{R}_{1}\right|\right),
\end{aligned}
$$

where $R_{1}$ is the remainder term in (2.2). 
Note that by Young's product inequality, we have

$$
\begin{aligned}
\iint \mathrm{d} q_{1} \mathrm{~d} p_{1}\left|p_{1}\right| m_{N, t}^{(1)}\left(q_{1}, p_{1}\right) & \leq \iint \mathrm{d} q_{1} \mathrm{~d} p_{1}\left(1+\left|p_{1}\right|^{2}\right) m_{N, t}^{(1)}\left(q_{1}, p_{1}\right) \\
& \leq(2 \pi)^{3}\left(1+2\left\langle\psi_{N}, \frac{\mathcal{K}}{N} \psi_{N}\right\rangle+C t^{2}\right)
\end{aligned}
$$

where we used (2.17) and Lemma 2.6 in the last inequality. Next, we want to bound the term associated with $\mathcal{R}_{1}$,

$$
\iint \mathrm{d} q_{1} \mathrm{~d} p_{1}\left|\mathcal{R}_{1}\right| \leq \hbar \iint \mathrm{d} q_{1} \mathrm{~d} p_{1}\left|\left\langle\nabla_{q_{1}} a\left(f_{q_{1}, p_{1}}^{\hbar}\right) \psi_{N, t}, a\left(f_{q_{1}, p_{1}}^{\hbar}\right) \psi_{N, t}\right\rangle\right| .
$$

Observe that we have,

$$
\begin{aligned}
& \hbar \iint \mathrm{d} q_{1} \mathrm{~d} p_{1}\left|\left\langle\nabla_{q_{1}} a\left(f_{q_{1}, p_{1}}^{\hbar}\right) \psi_{N, t}, a\left(f_{q_{1}, p_{1}}^{\hbar}\right) \psi_{N, t}\right\rangle\right| \\
& \leq \hbar \iint \mathrm{d} q_{1} \mathrm{~d} p_{1}\left\|\nabla_{q_{1}} a\left(f_{q_{1}, p_{1}}^{\hbar}\right) \psi_{N, t},\right\|\left\|a\left(f_{q_{1}, p_{1}}^{\hbar}\right) \psi_{N, t}\right\| \\
& \leq \hbar\left[\iint \mathrm{d} q_{1} \mathrm{~d} p_{1}\left\langle\nabla_{q_{1}} a\left(f_{q_{1}, p_{1}}^{\hbar}\right) \psi_{N, t}, \nabla_{q_{1}} a\left(f_{q_{1}, p_{1}}^{\hbar}\right) \psi_{N, t}\right\rangle\right]^{\frac{1}{2}} \\
& \quad\left[\iint \mathrm{d} q_{1} \mathrm{~d} p_{1}\left\langle\psi_{N, t}, a^{*}\left(f_{q_{1}, p_{1}}^{\hbar}\right) a\left(f_{q_{1}, p_{1}}^{\hbar}\right) \psi_{N, t}\right\rangle\right]^{\frac{1}{2}} \\
& \quad=\left[\hbar^{2} \iint \mathrm{d} q_{1} \mathrm{~d} p_{1} \iint \mathrm{d} w \mathrm{~d} u \nabla_{q_{1}} f_{q_{1}, p_{1}}^{\hbar}(w) \cdot \nabla_{q_{1}} \overline{f_{q_{1}, p_{1}}^{\hbar}(u)}\left\langle\psi_{N, t}, a_{w}^{*} a_{u} \psi_{N, t}\right\rangle\right]^{\frac{1}{2}}(2 \pi)^{\frac{3}{2}} \\
& \quad \leq(2 \pi)^{3} \sqrt{\hbar}\left[\int \mathrm{d} \widetilde{q}|\nabla f(\widetilde{q})|^{2}\right]^{\frac{1}{2}},
\end{aligned}
$$

where we used (2.15), Lemma 2.2. Thus, we have that

$$
\partial_{t} \iint \mathrm{d} q_{1} \mathrm{~d} p_{1}\left|q_{1}\right| m_{N, t}^{(1)}\left(q_{1}, p_{1}\right) \leq(2 \pi)^{3}\left(1+2\left\langle\psi_{N}, \frac{\mathcal{K}}{N} \psi_{N}\right\rangle+C t^{2}+C \sqrt{\hbar}\right) \leq C\left(1+t^{2}\right) .
$$

which gives the estimate for first moment after integrating with respect to time $t$.

We now consider the case of $2 \leq k \leq N$. In this computation, we make use of the properties of $k$-particle Husimi measure. Namely, that the $m_{N, t}^{(k)}$ is symmetric and satisfies the following equation

$$
\begin{aligned}
\frac{1}{(2 \pi)^{3}} \iint \mathrm{d} q_{k} \mathrm{~d} p_{k} m_{N, t}^{(k)}\left(q_{1}, p_{1}, \ldots, q_{k}, p_{k}\right) & =\frac{(N-k+1)}{N} m_{N, t}^{(k-1)}\left(q_{1}, p_{1}, \ldots, q_{k-1}, p_{k-1}\right) \\
& \leq m_{N, t}^{(k-1)}\left(q_{1}, p_{1}, \ldots, q_{k-1}, p_{k-1}\right) .
\end{aligned}
$$

Observe that for fixed $1 \leq k \leq N$.

$$
\begin{aligned}
& \int \cdots \int(\mathrm{d} q \mathrm{~d} p)^{\otimes k} \sum_{j=1}^{k}\left|p_{j}\right|^{2} m_{N, t}^{(k)}\left(q_{1}, p_{1}, \ldots, q_{k}, p_{k}\right) \\
& \quad=\sum_{j=1}^{k} \iint \mathrm{d} q_{j} \mathrm{~d} p_{j}\left|p_{j}\right|^{2} \int \cdots \int \mathrm{d} q_{1} \mathrm{~d} p_{1} \cdots \widehat{\mathrm{d} q_{j} \mathrm{~d} p_{j}} \cdots \mathrm{d} q_{k} \mathrm{~d} p_{k} m_{N, t}^{(k)}\left(q_{1}, p_{1}, \ldots, q_{k}, p_{k}\right) .
\end{aligned}
$$


Then, by using the symmetricity of $m_{N, t}^{(k)}$ and change of variables, we get

$$
\begin{aligned}
& =k \iint \mathrm{d} q \mathrm{~d} p|p|^{2} \int \cdots \int(\mathrm{d} q \mathrm{~d} p)^{\otimes k-1} m_{N, t}^{(k)}\left(q, p, q_{1}, p_{1} \ldots, q_{k-1}, p_{k-1}\right) \\
& =(2 \pi)^{3(k-1)} k \frac{(N-1) \cdots(N-k+1)}{N^{k-1}} \iint \mathrm{d} q \mathrm{~d} p|p|^{2} m_{N, t}^{(1)}(q, p) \\
& \leq(2 \pi)^{3 k} k\left(1+2\left\langle\psi_{N}, \frac{\mathcal{K}}{N} \psi_{N}\right\rangle+C t^{2}\right) \leq C\left(1+t^{2}\right),
\end{aligned}
$$

where we denoted $(\mathrm{d} q \mathrm{~d} p)^{\otimes k-1}=\mathrm{d} q_{1} \mathrm{~d} p_{1} \cdots \mathrm{d} q_{k-1} \mathrm{~d} p_{k-1}$.

Similar strategy is used to obtain the first moment with respect to $\mathbf{q}_{k}$. That is

$$
\begin{aligned}
& \int \cdots \int(\mathrm{d} q \mathrm{~d} p)^{\otimes k} \sum_{j=1}^{k}\left|q_{j}\right| m_{N, t}^{(k)}\left(q_{1}, p_{1}, \ldots, q_{k}, p_{k}\right) \\
& =(2 \pi)^{3(k-1)} k \frac{(N-1) \cdots(N-k+1)}{N^{k-1}} \iint \mathrm{d} q \mathrm{~d} p|q| m_{N, t}^{(1)}(q, p) \\
& \leq(2 \pi)^{3(k-1)} k \iint \mathrm{d} q \mathrm{~d} p|q| m_{N, t}^{(1)}(q, p) \leq C\left(1+t^{3}\right) .
\end{aligned}
$$

This yields the desired conclusion.

\subsection{Uniform Estimates for the Remainder Terms}

In this subsection, we give uniform estimates for the error terms that appear in (2.1) and (2.3). They are all bounded of order $\hbar^{\frac{1}{2}-\delta}$ for arbitrary small $\delta>0$. The proofs of all the following propositions will be provided in Sect. 3.2.

Proposition 2.4 Let Assumption A1 holds, then for $1 \leq k \leq N$, we have the following bound for $\mathcal{R}_{k}$ in (2.1) and (2.3). For arbitrary small $\delta>0$, the following estimate holds for any test function $\Phi \in C_{0}^{\infty}\left(\mathbb{R}^{6 k}\right)$,

$$
\left|\int \ldots \int(\mathrm{d} q \mathrm{~d} p)^{\otimes k} \Phi\left(q_{1}, p_{1}, \ldots, q_{k}, p_{k}\right) \nabla_{\mathbf{q}_{k}} \cdot \mathcal{R}_{k}\right| \leq C \hbar^{\frac{1}{2}-\delta},
$$

where $C$ depends on $\left\|D^{s(\delta)} \Phi\right\|_{\infty}$ and $k$.

Proposition 2.5 Let Assumption A1 and A2 hold, then we have the following bound for $\widetilde{\mathcal{R}}_{1}$ in (2.2). For arbitrary small $\delta>0$, the following estimate holds for any test function $\Phi \in C_{0}^{\infty}\left(\mathbb{R}^{6}\right)$,

$$
\left|\iint \mathrm{d} q_{1} \mathrm{~d} p_{1} \Phi\left(q_{1}, p_{1}\right) \nabla_{p_{1}} \cdot \widetilde{\mathcal{R}}_{1}\right| \leq C \hbar^{\frac{1}{2}-\delta},
$$

where $C$ depends on $\left\|D^{s(\delta)} \Phi\right\|_{\infty}$.

Proposition 2.6 Suppose that Assumption A1 and A2 hold. Denote the remainders terms $\widetilde{\mathcal{R}}_{k}$ and $\widehat{\mathcal{R}}_{k}$ as in (2.4). Then for $1 \leq k \leq N$ and arbitrary small $\delta>0$, the following estimates hold for any test function $\Phi \in C_{0}^{\infty}\left(\mathbb{R}^{6 k}\right)$,

$$
\left|\int \cdots \int(\mathrm{d} q \mathrm{~d} p)^{\otimes k} \Phi\left(q_{1}, p_{1}, \ldots, q_{k}, p_{k}\right) \cdot \widehat{\mathcal{R}}_{k}\right| \leq C \hbar^{3-\delta},
$$


and

$$
\left|\int \cdots \int(\mathrm{d} q \mathrm{~d} p)^{\otimes k} \Phi\left(q_{1}, p_{1}, \ldots, q_{k}, p_{k}\right) \nabla_{\mathbf{p}_{k}} \cdot \widetilde{\mathcal{R}}_{k}\right| \leq C \hbar^{\frac{1}{2}-\delta},
$$

where $C$ depends on $\left\|D^{s(\delta)} \Phi\right\|_{\infty}$ and $k$.

\subsection{Convergence to Infinite Hierarchy}

In this subsection, we prove that the $k$-particle Husimi measure $m_{N, t}^{(k)}$ has subsequence that converges weakly (as $N \rightarrow \infty$ ) to a limit $m_{t}^{(k)}$ in $L^{1}$, which is a solution of the infinite hierarchy in the sense of distribution.

The weak compactness of $k$-particle Husimi measure $m_{N, t}^{(k)}$ can be proved by the use of Dunford-Pettis theorem. ${ }^{3}$ In particular, we have the following result.

Proposition 2.7 Let $\left\{m_{N, t}^{(k)}\right\}_{N \in \mathbb{N}}$ be the k-particle Husimi measure, then there exists a subsequence $\left\{m_{N_{j}, t}^{(k)}\right\}_{j \in \mathbb{N}}$ that converges weakly in $L^{1}\left(\mathbb{R}^{6 k}\right)$ to a function $(2 \pi)^{3 k} m_{t}^{(k)}$, i.e. for all $\varphi \in L^{\infty}\left(\mathbb{R}^{6 k}\right)$, it holds

$$
\frac{1}{(2 \pi)^{3 k}} \int \cdots \int(\mathrm{d} q \mathrm{~d} p)^{\otimes k} m_{N_{j}, t}^{(k)} \varphi \rightarrow \int \cdots \int(\mathrm{d} q \mathrm{~d} p)^{\otimes k} m_{t}^{(k)} \varphi,
$$

when $j \rightarrow \infty$ for arbitrary fixed $k \geq 1$.

Proof To apply Dunford-Pettis theorem, we need to check that it is uniformly integrable and bounded. From the previous uniform estimates that we have obtained for $m_{N, t}^{(k)}$ from (2.6) and its second finite moment in Proposition 2.3 imply

$$
\left\|m_{N, t}^{(k)}\right\|_{L^{\infty}} \leq 1, \quad\left\|\left(\left|\mathbf{q}_{k}\right|+\left|\mathbf{p}_{k}\right|\right) m_{N, t}^{(k)}\right\|_{L^{1}} \leq C(t) .
$$

where $\mathbf{q}_{k}:=\left(q_{1}, \ldots, q_{k}\right), \mathbf{p}_{k}:=\left(p_{1}, \ldots, p_{k}\right)$ and $C(t)$ is a time-dependent constant, we can check the uniform integrability. More precisely, for any $\varepsilon>0$, by taking $r=\varepsilon^{-1}(2 \pi)^{3 k} C(t)$ we have that

$$
\frac{1}{(2 \pi)^{3 k}} \int \cdots \int_{\left|\mathbf{q}_{k}\right|+\left|\mathbf{p}_{k}\right| \geq r}(\mathrm{~d} q \mathrm{~d} p)^{\otimes k} m_{N, t}^{(k)} \leq \frac{1}{r} \frac{1}{(2 \pi)^{3 k}} \int \ldots \int(\mathrm{d} q \mathrm{~d} p)^{\otimes k}\left(\left|\mathbf{q}_{k}\right|+\left|\mathbf{p}_{k}\right|\right) m_{N, t}^{(k)} \leq \varepsilon .
$$

Furthermore, for arbitrary $\varepsilon>0$, by taking $\delta=\varepsilon$, we have that for all $E \subset \mathbb{R}^{6 k}$ with $\operatorname{Vol}(E) \leq \delta$, it holds

$$
\int \cdots \int_{E} m_{N, t}^{(k)} \leq\left\|m_{N, t}^{(k)}\right\|_{\infty} \operatorname{Vol}(E) \leq \varepsilon,
$$

which means that there is no concentration for the $k$-particle Husimi measure.

It is shown in (2.9) that the boundedness of $k$-particle Husimi measure in $L^{1}$, i.e.

$$
\left\|m_{N, t}^{(k)}\right\|_{L^{1}} \leq(2 \pi)^{3 k} .
$$

Then applying directly Dunford-Pettis Theorem one obtain that $k$-particle Husimi measure is weakly compact in $L^{1}$.

3 See [13] for the treatment of uniform integerability. 
Proof (Proof of Theorem 1.1 and Corollary 1.1) Cantor's diagonal procedure shows that we can take the same convergent subsequence of $m_{N, t}^{(k)}$ for all $k \geq 1$. Then by the error estimates obtained in Propositions 2.4, 2.5, and 2.6, we can obtain that the limit satisfies the infinite hierarchy (1.15) in the sense of distribution, by directly taking the limit in the weak formulation of (2.1) and (2.3).

Observe that the estimates for the remainder terms also show that any convergent subsequence of $m_{N, t}^{(k)}$ converges weakly in $L^{1}$ to the solution of the infinite hierarchy. Therefore, if furthermore, the infinite hierarchy has a unique solution, then the sequence $m_{N, t}^{(k)}$ itself converges weakly to the solution of the infinite hierarchy.

As for Corollary 1.1, one only need to combine the facts that the infinite hierarchy has a unique solution and that the tensor products of the solution of the Vlasov equation (1.13), $m_{t}^{\otimes k}$ is a solution of the infinite hierarchy.

Lastly, by Theorem 7.12 in [47], we would obtain the convergence in 1-Wasserstein metric.

\section{Completion of the Reformulation and Estimates in the Proof}

\subsection{Proof of the Reformulation in Sect. 2.1}

In this subsection we supply the proofs for the reformulation of Schrödinger equation into a hierarchy of $k(1 \leq k \leq N)$ particle Husimi measure. The reformulation shares similar structure to the classical BBGKY hierarchy.

Proof (Proof of Proposition 2.1) First, observe that taking the time derivative on the Husimi measure, we have

$$
\begin{aligned}
& 2 \mathrm{i} \hbar \partial_{t} m_{N, t}^{(1)}\left(q_{1}, p_{1}\right) \\
& =\left(\hbar^{2} \iiint \mathrm{d} w \mathrm{~d} u \mathrm{~d} x f_{q_{1}, p_{1}}^{\hbar}(w) \overline{f_{q_{1}, p_{1}}^{\hbar}(u)}\left\langle\psi_{N, t}, a_{w}^{*} a_{u} \nabla_{x} a_{x}^{*} \nabla_{x} a_{x} \psi_{N, t}\right\rangle\right. \\
& \left.-\hbar^{2} \iiint \mathrm{d} w \mathrm{~d} u \mathrm{~d} x \overline{f_{q_{1}, p_{1}}^{\hbar}(w)} f_{q_{1}, p_{1}}^{\hbar}(u)\left\langle\psi_{N, t}, \nabla_{x} a_{x}^{*} \nabla_{x} a_{x} a_{u}^{*} a_{w} \psi_{N, t}\right\rangle\right) \\
& +\left(\frac{1}{N} \iint \mathrm{d} w \mathrm{~d} u \iint \mathrm{d} x \mathrm{~d} y f_{q_{1}, p_{1}}^{\hbar}(w) \overline{f_{q_{1}, p_{1}}^{\hbar}(u)}\left\langle\psi_{N, t}, V(x-y) a_{w}^{*} a_{u} a_{x}^{*} a_{y}^{*} a_{y} a_{x} \psi_{N, t}\right\rangle\right. \\
& \left.-\frac{1}{N} \iint \mathrm{d} w \mathrm{~d} u \iint \mathrm{d} x \mathrm{~d} y \overline{f_{q_{1}, p_{1}}^{\hbar}(w)} f_{q_{1}, p_{1}}^{\hbar}(u)\left\langle\psi_{N, t}, V(x-y) a_{x}^{*} a_{y}^{*} a_{y} a_{x} a_{u}^{*} a_{w} \psi_{N, t}\right\rangle\right) \\
& =: I_{1}+I_{1} \text {. }
\end{aligned}
$$

Now, focus on $I_{1}$, we have

$$
\begin{aligned}
I_{1}= & \hbar^{2} \iiint \mathrm{d} w \mathrm{~d} u \mathrm{~d} x f_{q_{1}, p_{1}}^{\hbar}(w) \overline{f_{q_{1}, p_{1}}^{\hbar}(u)}\left\langle\psi_{N, t}, a_{w}^{*} a_{u} \nabla_{x} a_{x}^{*} \nabla_{x} a_{x} \psi_{N, t}\right\rangle \\
& -\hbar^{2} \iiint \mathrm{d} w \mathrm{~d} u \mathrm{~d} x f_{q_{1}, p_{1}}^{\hbar}(w) \overline{f_{q_{1}, p_{1}}^{\hbar}(u)}\left\langle\psi_{N, t}, \nabla_{x} a_{x}^{*} \nabla_{x} a_{x} a_{w}^{*} a_{u} \psi_{N, t}\right\rangle,
\end{aligned}
$$

where the last equality is just change of variable on the complex conjugate term. Then, from CAR, observe we have that 


$$
\begin{aligned}
-a_{w}^{*} a_{u} a_{x}^{*} \Delta_{x} a_{x} & =a_{w}^{*} a_{x}^{*} a_{u} \Delta_{x} a_{x}-\delta_{u=x} a_{w}^{*} \Delta_{x} a_{x} \\
& =a_{x}^{*} a_{w}^{*} \Delta_{x} a_{x} a_{u}-\delta_{u=x} a_{w}^{*} \Delta_{x} a_{x} \\
& =\Delta_{x} a_{x}^{*} a_{w}^{*} a_{x} a_{u}-\delta_{u=x} a_{w}^{*} \Delta_{x} a_{x} \\
& =-\Delta_{x} a_{x}^{*} a_{x} a_{w}^{*} a_{u}+\delta_{w=x} \Delta_{x} a_{x}^{*} a_{u}-\delta_{u=x} a_{w}^{*} \Delta_{x} a_{x},
\end{aligned}
$$

where integration by parts and CAR of the operator have been used several times. Putting this back, we cancel out the the second term and get

$$
\begin{aligned}
I_{1}= & \hbar^{2} \iiint \mathrm{d} w \mathrm{~d} u \mathrm{~d} x f_{q_{1}, p_{1}}^{\hbar}(w) \overline{f_{q_{1}, p_{1}}^{\hbar}(u)}\left\langle\psi_{N, t},\left(\delta_{w=x} \Delta_{x} a_{x}^{*} a_{u}-\delta_{u=x} a_{w}^{*} \Delta_{x} a_{x}\right) \psi_{N, t}\right\rangle \\
= & \hbar^{2} \iint \mathrm{d} w \mathrm{~d} u\left(\Delta_{w} f_{q_{1}, p_{1}}^{\hbar}(w)\right) \overline{f_{q_{1}, p_{1}}^{\hbar}(u)}\left\langle\psi_{N, t}, a_{w}^{*} a_{u} \psi_{N, t}\right\rangle \\
& -\hbar^{2} \iint \mathrm{d} w \mathrm{~d} u f_{q_{1}, p_{1}}^{\hbar}(w)\left(\Delta_{u} \overline{f_{q_{1}, p_{1}}^{\hbar}(u)}\right)\left\langle\psi_{N, t}, a_{w}^{*} a_{u} \psi_{N, t}\right\rangle .
\end{aligned}
$$

Now, observe the following

$$
\begin{aligned}
\nabla_{u} \overline{f_{q_{1}, p_{1}}^{\hbar}(u)} & =\nabla_{u}\left(\hbar^{-\frac{3}{4}} f\left(\frac{u-q_{1}}{\sqrt{\hbar}}\right) e^{-\frac{\mathrm{i}}{\hbar} p_{1} \cdot u}\right) \\
& =\hbar^{-\frac{3}{4}} \nabla_{u} f\left(\frac{u-q_{1}}{\sqrt{\hbar}}\right) e^{-\frac{\mathrm{i}}{\hbar} p_{1} \cdot u}+\hbar^{-\frac{3}{4}} f\left(\frac{u-q_{1}}{\sqrt{\hbar}}\right) \nabla_{u} e^{-\frac{\mathrm{i}}{\hbar} p_{1} \cdot u} \\
& =-\hbar^{-\frac{3}{4}} \nabla_{q_{1}} f\left(\frac{u-q_{1}}{\sqrt{\hbar}}\right) e^{-\frac{\mathrm{i}}{\hbar} p_{1} \cdot u}-\mathrm{i} \hbar^{-1} p_{1} \cdot \hbar^{-\frac{3}{4}} f\left(\frac{u-q_{1}}{\sqrt{\hbar}}\right) e^{-\frac{\mathrm{i}}{\hbar} p_{1} \cdot u} \\
& =\left(-\nabla_{q_{1}}-\mathrm{i} \hbar^{-1} p_{1}\right) \overline{f_{q_{1}, p_{1}}^{\hbar}(u)}
\end{aligned}
$$

and furthermore,

$$
\begin{aligned}
\Delta_{u} \overline{f_{q_{1}, p_{1}}^{\hbar}(u)} & =\nabla_{u} \cdot \nabla_{u} \overline{f_{q_{1}, p_{1}}^{\hbar}(u)} \\
& =\nabla_{u} \cdot\left(-\nabla_{q_{1}}-\mathrm{i} \hbar^{-1} p_{1}\right) \overline{f_{q_{1}, p_{1}}^{\hbar}(u)} \\
& =\left(-\nabla_{q_{1}}-\mathrm{i} \hbar^{-1} p_{1}\right) \cdot\left(-\nabla_{q_{1}}-\mathrm{i} \hbar^{-1} p_{1}\right) \overline{f_{q_{1}, p_{1}}^{\hbar}(u)} \\
& =\left(\Delta_{q_{1}}+2 \mathrm{i} \hbar^{-1} p_{1} \cdot \nabla_{q_{1}}-\hbar^{-2} p_{1}^{2}\right) \overline{f_{q_{1}, p_{1}}^{\hbar}(u)} .
\end{aligned}
$$

and similarly

$$
\Delta_{w} f_{q_{1}, p_{1}}^{\hbar}(w)=\left(\Delta_{q_{1}}-2 \mathrm{i} \hbar^{-1} p_{1} \cdot \nabla_{q_{1}}-\hbar^{-2} p_{1}^{2}\right) f_{q_{1}, p_{1}}^{\hbar}(w),
$$


we obtain by putting these back into (3.1),

$$
\begin{aligned}
I_{1}= & \hbar^{2}\left[\left\langle\Delta_{q_{1}} \int \mathrm{d} w \overline{f_{q_{1}, p_{1}}^{\hbar}(w)} a_{w} \psi_{N, t}, \int \mathrm{d} u \overline{f_{q_{1}, p_{1}}^{\hbar}(u)} a_{u} \psi_{N, t}\right\rangle\right. \\
& \left.-\left\langle\int \mathrm{d} w \overline{f_{q_{1}, p_{1}}^{\hbar}(w)} a_{w} \psi_{N, t}, \Delta_{q_{1}} \int \mathrm{d} u \overline{f_{q_{1}, p_{1}}^{\hbar}(u)} a_{u} \psi_{N, t}\right\rangle\right] \\
& -2 \mathrm{i} \hbar p_{1} \cdot\left[\left\langle\nabla_{q_{1}} \int \mathrm{d} w \overline{f_{q_{1}, p_{1}}^{\hbar}(w)} a_{w} \psi_{N, t}, \int \mathrm{d} u \overline{f_{q_{1}, p_{1}}^{\hbar}(u)} a_{u} \psi_{N, t}\right\rangle\right. \\
& \left.+\left\langle\int \mathrm{d} w \overline{f_{q_{1}, p_{1}}^{\hbar}(w)} a_{w} \psi_{N, t}, \nabla_{q_{1}} \int \mathrm{d} u \overline{f_{q_{1}, p_{1}}^{\hbar}(u)} a_{u} \psi_{N, t}\right\rangle\right] \\
= & 2 \mathrm{i} \hbar^{2} \operatorname{Im}\left\langle\Delta_{q_{1}} a\left(f_{q_{1}, p_{1}}^{\hbar}\right) \psi_{N, t}, a\left(f_{q_{1}, p_{1}}^{\hbar}\right) \psi_{N, t}\right\rangle-2 \mathrm{i} \hbar p_{1} \cdot \nabla_{q_{1}} m_{N, t}^{(1)}\left(q_{1}, p_{1}\right) .
\end{aligned}
$$

Since the Husimi measure is actually a real-valued function, we have that

$$
\partial_{t} m_{N, t}^{(1)}\left(q_{1}, p_{1}\right)+p_{1} \cdot \nabla_{q_{1}} m_{N, t}^{(1)}\left(q_{1}, p_{1}\right)=\operatorname{Re}\left(\frac{I I_{1}}{2 \mathrm{i} \hbar}\right)+\hbar \operatorname{Im}\left\langle\Delta_{q_{1}} a\left(f_{q_{1}, p_{1}}^{\hbar}\right) \psi_{N, t}, a\left(f_{q_{1}, p_{1}}^{\hbar}\right) \psi_{N, t}\right\rangle .
$$

Now, we turn our focus on $I_{1}$, i.e.,

$$
\begin{aligned}
I I_{1}= & \frac{1}{N} \iint \mathrm{d} w \mathrm{~d} u \iint \mathrm{d} x \mathrm{~d} y f_{q_{1}, p_{1}}^{\hbar}(w) \overline{f_{q_{1}, p_{1}}^{\hbar}(u)} \\
& \left\langle\psi_{N, t}, V(x-y) a_{w}^{*} a_{u} a_{x}^{*} a_{y}^{*} a_{y} a_{x} \psi_{N, t}\right\rangle \\
& -\frac{1}{N} \iint \mathrm{d} w \mathrm{~d} u \iint \mathrm{d} x \mathrm{~d} y \overline{f_{q_{1}, p_{1}}^{\hbar}(w)} f_{q_{1}, p_{1}}^{\hbar}(u) \\
& \left\langle\psi_{N, t}, V(x-y) a_{x}^{*} a_{y}^{*} a_{y} a_{x} a_{u}^{*} a_{w} \psi_{N, t}\right\rangle .
\end{aligned}
$$

Observe that

$$
\begin{aligned}
a_{w}^{*} a_{u} a_{x}^{*} a_{y}^{*} a_{y} a_{x}= & a_{x}^{*} a_{y}^{*} a_{y} a_{x} a_{w}^{*} a_{u} \\
& +\delta_{w=y} a_{x}^{*} a_{y}^{*} a_{x} a_{u}-\delta_{w=x} a_{x}^{*} a_{y}^{*} a_{y} a_{u} \\
& +\delta_{u=x} a_{w}^{*} a_{y}^{*} a_{y} a_{x}-\delta_{u=y} a_{w}^{*} a_{x}^{*} a_{y} a_{x} .
\end{aligned}
$$

The first term and the complex conjugate term vanishes under changes of variable, $u$ to $w$ and $w$ to $u$. Therefore, since from assumption $V(x)=V(-x)$, we have 


$$
\begin{aligned}
I I_{1}= & \frac{1}{N} \iiint \mathrm{d} w \mathrm{~d} u \mathrm{~d} x f_{q_{1}, p_{1}}^{\hbar}(w) \overline{f_{q_{1}, p_{1}}^{\hbar}(u)}\left\langle\psi_{N, t}, V(x-w) a_{x}^{*} a_{w}^{*} a_{x} a_{u} \psi_{N, t}\right\rangle \\
& -\frac{1}{N} \iiint \mathrm{d} w \mathrm{~d} u \mathrm{~d} x f_{q_{1}, p_{1}}^{\hbar}(w) \overline{f_{q_{1}, p_{1}}^{\hbar}(u)}\left\langle\psi_{N, t}, V(x-u) a_{w}^{*} a_{x}^{*} a_{u} a_{x} \psi_{N, t}\right\rangle \\
& +\frac{1}{N} \iiint \mathrm{d} w \mathrm{~d} u \mathrm{~d} y f_{q_{1}, p_{1}}^{\hbar}(w) \overline{f_{q_{1}, p_{1}}^{\hbar}(u)}\left\langle\psi_{N, t}, V(u-y) a_{w}^{*} a_{y}^{*} a_{y} a_{u} \psi_{N, t}\right\rangle \\
& -\frac{1}{N} \iiint \mathrm{d} w \mathrm{~d} u \mathrm{~d} y f_{q_{1}, p_{1}}^{\hbar}(w) \overline{f_{q_{1}, p_{1}}^{\hbar}(u)}\left\langle\psi_{N, t}, V(w-y) a_{w}^{*} a_{y}^{*} a_{y} a_{u} \psi_{N, t}\right\rangle \\
= & \frac{1}{N} \iiint \mathrm{d} w \mathrm{~d} u \mathrm{~d} x f_{q_{1}, p_{1}}^{\hbar}(w) \overline{f_{q_{1}, p_{1}}^{\hbar}(u)}(V(u-x)-V(w-x))\left\langle\psi_{N, t}, a_{w}^{*} a_{x}^{*} a_{x} a_{u} \psi_{N, t}\right\rangle \\
& +\frac{1}{N} \iiint \mathrm{d} w \mathrm{~d} u \mathrm{~d} y f_{q_{1}, p_{1}}^{\hbar}(w) \overline{f_{q_{1}, p_{1}}^{\hbar}(u)}(V(u-y)-V(w-y))\left\langle\psi_{N, t}, a_{w}^{*} a_{y}^{*} a_{y} a_{u} \psi_{N, t}\right\rangle \\
= & \frac{2}{N} \iiint \mathrm{d} w \mathrm{~d} u \mathrm{~d} y f_{q_{1}, p_{1}}^{\hbar}(w) \overline{f_{q_{1}, p_{1}}^{\hbar}(u)}(V(u-y)-V(w-y))\left\langle\psi_{N, t}, a_{w}^{*} a_{y}^{*} a_{y} a_{u} \psi_{N, t}\right\rangle .
\end{aligned}
$$

Now, note that mean value theorem gives

$$
V(u-y)-V(w-y)=\int_{0}^{1} \mathrm{~d} s \nabla V(s(u-y)+(1-s)(w-y)) \cdot(u-w),
$$

and observe that since, $V(s(u-y)+(1-s)(w-y))=V(s u+(1-s) w-y)$, we can have from (3.6) the following

$$
\begin{aligned}
I I_{1}= & \frac{2}{N} \iiint \mathrm{d} w \mathrm{~d} u \mathrm{~d} y f_{q_{1}, p_{1}}^{\hbar}(w) \overline{f_{q_{1}, p_{1}}^{\hbar}(u)}\left(\int_{0}^{1} \mathrm{~d} s \nabla V(s u+(1-s) w-y)\right) \\
& \cdot(u-w) \cdot \quad\left\langle\psi_{N, t}, a_{w}^{*} a_{y}^{*} a_{y} a_{u} \psi_{N, t}\right\rangle \\
= & \frac{2 \mathrm{i} \hbar}{N} \iiint \mathrm{d} w \mathrm{~d} u \mathrm{~d} y \int_{0}^{1} \nabla V(s u+(1-s) w-y) \\
& \cdot \nabla_{p_{1}}\left(f_{q_{1}, p_{1}}^{\hbar}(w) \overline{f_{q_{1}, p_{1}}^{\hbar}(u)}\right)\left\langle\psi_{N, t}, a_{w}^{*} a_{y}^{*} a_{y} a_{u} \psi_{N, t}\right\rangle \\
= & \frac{2 \mathrm{i} \hbar}{N} \iiint \mathrm{d} w \mathrm{~d} u \mathrm{~d} y \int_{0}^{1} \nabla V(s u+(1-s) w-y) \\
& \cdot \nabla_{p_{1}}\left(f_{q_{1}, p_{1}}^{\hbar}(w) \overline{f_{q_{1}, p_{1}}^{\hbar}(u)}\right)\left\langle a_{w} a_{y} \psi_{N, t}, a_{u} a_{y} \psi_{N, t}\right\rangle,
\end{aligned}
$$

where we use the fact that

$$
\nabla_{p_{1}}\left(f_{q_{1}, p_{1}}^{\hbar}(w) \overline{f_{q_{1}, p_{1}}^{\hbar}(u)}\right)=\frac{\mathrm{i}}{\hbar}(w-u) \cdot f_{q_{1}, p_{1}}^{\hbar}(w) \overline{f_{q_{1}, p_{1}}^{\hbar}(u)} .
$$

Then we get

$$
I I_{1}=\frac{2 \mathrm{i} \hbar}{N} \iiint \mathrm{d} w \mathrm{~d} u \mathrm{~d} y \int_{0}^{1} \nabla V(s u+(1-s) w-y) \cdot \nabla_{p_{1}}\left(f_{q_{1}, p_{1}}^{\hbar}(w) \overline{f_{q_{1}, p_{1}}^{\hbar}(u)}\right)\left\langle a_{w} a_{y} \psi_{N, t}, a_{u} a_{y} \psi_{N, t}\right\rangle .
$$

Applying the following projection

$$
\frac{1}{(2 \pi \hbar)^{3}} \iint \mathrm{d} q_{2} \mathrm{~d} p_{2}\left|f_{q_{2}, p_{2}}^{\hbar}\right\rangle\left\langle f_{q_{2}, p_{2}}^{\hbar}\right|=\mathbb{1},
$$

onto $a_{y} \psi_{N, t}$, we get

$$
a_{y} \psi_{N, t}=\frac{1}{(2 \pi \hbar)^{3}} \iint \mathrm{d} q_{2} \mathrm{~d} p_{2} f_{q_{2}, p_{2}}^{\hbar}(y) \int \mathrm{d} v \overline{f_{q_{2}, p_{2}}^{\hbar}(v)} a_{v} \psi_{N, t} .
$$


Putting this back into (3.10), we get the following

$$
\begin{array}{r}
I I_{1}=\frac{2 \mathrm{i} \hbar}{N} \frac{1}{(2 \pi \hbar)^{3}} \iint \mathrm{d} w \mathrm{~d} u \iint \mathrm{d} y \mathrm{~d} v \iint \mathrm{d} q_{2} \mathrm{~d} p_{2} \int_{0}^{1} \mathrm{~d} s \nabla V(s u+(1-s) w-y) \\
\cdot \nabla_{p_{1}}\left(f_{q_{1}, p_{1}}^{\hbar}(w) \overline{f_{q_{1}, p_{1}}^{\hbar}(u)}\right) f_{q_{2}, p_{2}}^{\hbar}(y) \overline{f_{q_{2}, p_{2}}^{\hbar}(v)}\left\langle a_{w} a_{y} \psi_{N, t}, a_{u} a_{v} \psi_{N, t}\right\rangle .
\end{array}
$$

Recall that $\hbar^{3}=N^{-1}$, we have

$$
\begin{aligned}
I_{1}=\frac{2 \mathrm{i} \hbar}{(2 \pi)^{3}} \iint \mathrm{d} w \mathrm{~d} u \iint \mathrm{d} y \mathrm{~d} v \iint \mathrm{d} q_{2} \mathrm{~d} p_{2} \int_{0}^{1} \mathrm{~d} s \nabla V(s u+(1-s) w-y) \\
\cdot \nabla_{p_{1}}\left(f_{q_{1}, p_{1}}^{\hbar}(w) \overline{f_{q_{1}, p_{1}}^{\hbar}(u)}\right) f_{q_{2}, p_{2}}^{\hbar}(y) \overline{f_{q_{2}, p_{2}}^{\hbar}(v)}\left\langle a_{w} a_{y} \psi_{N, t}, a_{u} a_{v} \psi_{N, t}\right\rangle .
\end{aligned}
$$

Therefore, we have the last term in (3.5) as

$$
\begin{aligned}
\operatorname{Re} \frac{I I_{1}}{2 i \hbar}=\frac{1}{(2 \pi)^{3}} \operatorname{Re} \iint \mathrm{d} w \mathrm{~d} u \iint \mathrm{d} y \mathrm{~d} v \iint \mathrm{d} q_{2} \mathrm{~d} p_{2} \int_{0}^{1} \mathrm{~d} s \nabla V(s u+(1-s) w-y) \\
\cdot \nabla_{p_{1}}\left(f_{q_{1}, p_{1}}^{\hbar}(w) \overline{f_{q_{1}, p_{1}}^{\hbar}(u)}\right) f_{q_{2}, p_{2}}^{\hbar}(y) \overline{f_{q_{2}, p_{2}}^{\hbar}(v)}\left\langle a_{w} a_{y} \psi_{N, t}, a_{u} a_{v} \psi_{N, t}\right\rangle,
\end{aligned}
$$

thus we have derived the equation for $m_{N, t}^{(1)}\left(q_{1}, p_{1}\right)$.

We have proved the reformulation from Schrödinger equation into 1-particle Husimi measure. We also observed that it contains a resemblance to the classical Vlasov equation. Next we want to prove the similar result for $2 \leq k \leq N$.

Proof (Proof of Proposition 2.2) Now we focus on the case where $2 \leq k \leq N$. As in the proof for the case of $k=1$, we first observe that for every $k \in \mathbb{N}$,

$$
\begin{aligned}
2 \mathrm{i} \hbar \partial_{t} & m_{N, t}^{(k)}\left(q_{1}, p_{1}, \ldots, q_{k}, p_{k}\right) \\
= & \left(-\hbar^{2} \int \cdots \int(\mathrm{d} w \mathrm{~d} u)^{\otimes k} \int \mathrm{d} x\left(f_{q, p}^{\hbar}(w) \overline{f_{q, p}^{\hbar}(u)}\right)^{\otimes k} \Delta_{x}\left\langle\psi_{N, t}, a_{w_{1}}^{*} \cdots a_{w_{k}}^{*} a_{u_{k}} \cdots a_{u_{1}} a_{x}^{*} a_{x} \psi_{N, t}\right\rangle\right. \\
& \left.+\hbar^{2} \int \cdots \int(\mathrm{d} w \mathrm{~d} u)^{\otimes k} \int \mathrm{d} x\left(f_{q, p}^{\hbar}(w) \overline{f_{q, p}^{\hbar}(u)}\right)^{\otimes k} \Delta_{x}\left\langle\psi_{N, t}, a_{x}^{*} a_{x} a_{w_{1}}^{*} \cdots a_{w_{k}}^{*} a_{u_{k}} \cdots a_{u_{1}} \psi_{N, t}\right\rangle\right) \\
& +\left(\frac{1}{N} \int \cdots \int(\mathrm{d} w \mathrm{~d} u)^{\otimes k} \iint \mathrm{d} x \mathrm{~d} y V(x-y)\left(f_{q, p}^{\hbar}(w) \overline{f_{q, p}^{\hbar}(u)}\right)^{\otimes k}\right. \\
& \left\langle\psi_{N, t}, a_{w_{1}}^{*} \cdots a_{w_{k}}^{*} a_{u_{k}} \cdots a_{u_{1}} a_{x}^{*} a_{y}^{*} a_{y} a_{x} \psi_{N, t}\right\rangle \\
& -\frac{1}{N} \int \cdots \int(\mathrm{d} w \mathrm{~d} u)^{\otimes k} \iint \mathrm{d} x \mathrm{~d} y V(x-y)\left(f_{q, p}^{\hbar}(w) \overline{f_{q, p}^{\hbar}(u)}\right) \\
& \left.\left\langle\psi_{N, t}, a_{x}^{*} a_{y}^{*} a_{y} a_{x} a_{w_{1}}^{*} \cdots a_{w_{k}}^{*} a_{u_{k}} \cdots a_{u_{1}} \psi_{N, t}\right\rangle\right) \\
= & : I_{2}+I I_{2},
\end{aligned}
$$

where the tensor product denotes $(\mathrm{d} w \mathrm{~d} u)^{\otimes k}=\mathrm{d} w_{1} \cdots \mathrm{d} w_{k} \mathrm{~d} u_{1} \cdots \mathrm{d} u_{k}$. 
We first focus on the $I_{2}$ part of (3.14), i.e.,

$$
\begin{aligned}
I_{2}= & -\hbar^{2} \int \cdots \int(\mathrm{d} w \mathrm{~d} u)^{\otimes k} \int \mathrm{d} x\left(f_{q, p}^{\hbar}(w) \overline{f_{q, p}^{\hbar}(u)}\right)^{\otimes k} \Delta_{x}\left\langle\psi_{N, t}, a_{w_{1}}^{*} \cdots a_{w_{k}}^{*} a_{u_{k}} \cdots a_{u_{1}} a_{x}^{*} a_{x} \psi_{N, t}\right\rangle \\
& +\hbar^{2} \int \cdots \int(\mathrm{d} w \mathrm{~d} u)^{\otimes k} \int \mathrm{d} x\left(f_{q, p}^{\hbar}(w) \overline{f_{q, p}^{\hbar}(u)}\right)^{\otimes k} \Delta_{x}\left\langle\psi_{N, t}, a_{x}^{*} a_{x} a_{w_{1}}^{*} \cdots a_{w_{k}}^{*} a_{u_{k}} \cdots a_{u_{1}} \psi_{N, t}\right\rangle .
\end{aligned}
$$

Observe that we have

$$
\begin{aligned}
a_{w_{1}}^{*} \cdots a_{w_{k}}^{*} a_{u_{k}} \cdots a_{u_{1}} a_{x}^{*} a_{x}= & (-1)^{4 k} a_{x}^{*} a_{x} a_{w_{1}}^{*} \cdots a_{w_{k}}^{*} a_{u_{k}} \cdots a_{u_{1}} \\
& +a_{x}^{*}\left(\sum_{j=1}^{k}(-1)^{j} \delta_{x=w_{j}} a_{w_{1}}^{*} \cdots \widehat{a_{w_{j}}^{*}} \cdots a_{w_{k}}^{*}\right) a_{u_{k}} \cdots a_{u_{1}} \\
& -a_{w_{1}}^{*} \cdots a_{w_{k}}^{*}\left(\sum_{j=1}^{k}(-1)^{j} \delta_{x=u_{j}} a_{u_{k}} \cdots \widehat{a_{u_{j}}} \cdots a_{u_{1}}\right) a_{x},
\end{aligned}
$$

where the hat indicates exclusion of that element.

Putting this back into (3.15), we obtain

$$
\begin{gathered}
I_{2}=\hbar^{2} \int \ldots \int(\mathrm{d} w \mathrm{~d} u)^{\otimes k} \int \mathrm{d} x\left(f_{q, p}^{\hbar}(w) \overline{f_{q, p}^{\hbar}(u)}\right)^{\otimes k} \\
\cdot \Delta_{x}\left\langle\psi_{N, t}, a_{w_{1}}^{*} \cdots a_{w_{k}}^{*}\left(\sum_{j=1}^{k}(-1)^{j} \delta_{x=u_{j}} a_{u_{k}} \cdots \widehat{a_{u_{j}}} \cdots a_{u_{1}}\right) a_{x} \psi_{N, t}\right\rangle \\
-\hbar^{2} \int \ldots \int(\mathrm{d} w \mathrm{~d} u)^{\otimes k} \int \mathrm{d} x\left(f_{q, p}^{\hbar}(w) \overline{f_{q, p}^{\hbar}(u)}\right)^{\otimes k} \\
\cdot \Delta_{x}\left\langle\psi_{N, t}, a_{x}^{*}\left(\sum_{j=1}^{k}(-1)^{j} \delta_{x=w_{j}} a_{w_{1}}^{*} \cdots \widehat{a_{w_{j}}^{*}} \cdots a_{w_{k}}^{*}\right) a_{u_{k}} \cdots a_{u_{1}} \psi_{N, t}\right\rangle \\
=\hbar^{2} \sum_{j=1}^{k}(-1)^{j} \int \ldots /(\mathrm{d} w \mathrm{~d} u)^{\otimes k}\left(f_{q, p}^{\hbar}(w) \overline{f_{q, p}^{\hbar}(u)}\right)^{\otimes k} \\
\cdot\left(\Delta_{u_{j}}\left\langle\psi_{N, t}, a_{w_{1}}^{*} \cdots a_{w_{k}}^{*}\left(a_{u_{k}} \cdots \widehat{a_{u_{j}}} \cdots a_{u_{1}}\right) a_{u_{j}} \psi_{N, t}\right\rangle\right. \\
\left.-\Delta_{w_{j}}\left\langle\psi_{N, t}, a_{w_{j}}^{*}\left(a_{w_{1}}^{*} \cdots \widehat{a_{w_{j}}^{*}} \cdots a_{w_{k}}^{*}\right) a_{u_{k}} \cdots a_{u_{1}} \psi_{N, t}\right\rangle\right) .
\end{gathered}
$$

Note that, if we want to move the missing $a_{u_{j}}$ or $a_{w_{j}}^{*}$ back to their original position after applying the delta function, we have for fixed $j$

$$
\begin{aligned}
(-1)^{j} a_{w_{1}}^{*} \cdots a_{w_{k}}^{*}\left[a_{u_{k}} \cdots \widehat{a_{u_{j}}} \cdots a_{u_{1}}\right] a_{u_{j}} & =\frac{(-1)^{j}}{(-1)^{j-1}} a_{w_{1}}^{*} \cdots a_{w_{k}}^{*} a_{u_{k}} \cdots a_{u_{1}} \\
& =(-1)^{1} a_{w_{1}}^{*} \cdots a_{w_{k}}^{*} a_{u_{k}} \cdots a_{u_{1}} \\
(-1)^{j} a_{w_{j}}^{*}\left[a_{w_{1}}^{*} \cdots \widehat{a_{w_{j}}^{*}} \cdots a_{w_{k}}^{*}\right] a_{u_{k}} \cdots a_{u_{1}} & =(-1)^{1} a_{w_{1}}^{*} \cdots a_{w_{k}}^{*} a_{u_{k}} \cdots a_{u_{1}} .
\end{aligned}
$$


Therefore, continuing from (3.17), we have

$$
I_{2}=-\hbar^{2} \sum_{j=1}^{k} \int \cdots \int(\mathrm{d} w \mathrm{~d} u)^{\otimes k}\left(f_{q, p}^{\hbar}(w) \overline{f_{q, p}^{\hbar}(u)}\right)^{\otimes k}\left[\Delta_{u_{j}}-\Delta_{w_{j}}\right]\left\langle\psi_{N, t}, a_{w_{1}}^{*} \cdots a_{w_{k}}^{*} a_{u_{k}} \cdots a_{u_{1}} \psi_{N, t}\right\rangle .
$$

Now, by integration by parts on (3.18) and note that the Laplacian acting on the coherent state would be similar to (3.2) and (3.3), i.e., for fixed $j$ where $1 \leq j \leq k$

$$
\begin{aligned}
& \Delta_{u_{j}}\left(\overline{f_{q, p}^{\hbar}(u)}\right)^{\otimes k}=\left(\Delta_{q_{j}}+2 \mathrm{i} \hbar^{-1} p_{j} \cdot \nabla_{q_{j}}-\hbar^{-2} p_{j}^{2}\right)\left(\overline{f_{q, p}^{\hbar}(u)}\right)^{\otimes k}, \\
& \Delta_{w_{j}}\left(f_{q, p}^{\hbar}(w)\right)^{\otimes k}=\left(\Delta_{q_{j}}-2 \mathrm{i} \hbar^{-1} p_{j} \cdot \nabla_{q_{j}}-\hbar^{-2} p_{j}^{2}\right)\left(f_{q, p}^{\hbar}(w)\right)^{\otimes k} .
\end{aligned}
$$

Thus, we have similar for when $k=1$, the kinetic part as

$$
\begin{aligned}
I_{2}= & -2 \mathrm{i} \hbar \sum_{j=1}^{k} p_{j} \cdot \nabla_{q_{j}} \int \cdots \int(\mathrm{d} w \mathrm{~d} u)^{\otimes k}\left(f_{q, p}^{\hbar}(w) \overline{f_{q, p}^{\hbar}(u)}\right)^{\otimes k}\left\langle\psi_{N, t}, a_{w_{1}}^{*} \cdots a_{w_{k}}^{*} a_{u_{k}} \cdots a_{u_{1}} \psi_{N, t}\right\rangle \\
& +2 \hbar^{2} \operatorname{Im} \sum_{j=1}^{k}\left\langle\Delta_{q_{j}} a\left(f_{q_{k}, p_{k}}^{\hbar}\right) \cdots a\left(f_{q_{1}, p_{1}}^{\hbar}\right) \psi_{N, t}, a\left(f_{q_{k}, p_{k}}^{\hbar}\right) \cdots a\left(f_{q_{1}, p_{1}}^{\hbar}\right) \psi_{N, t}\right\rangle \\
= & -2 \mathrm{i} \hbar \mathbf{p}_{k} \cdot \nabla_{\mathbf{q}_{k}}\left\langle a\left(f_{q_{k}, p_{k}}^{\hbar}\right) \cdots a\left(f_{q_{1}, p_{1}}^{\hbar}\right) \psi_{N, t}, a\left(f_{q_{k}, p_{k}}^{\hbar}\right) \cdots a\left(f_{q_{1}, p_{1}}^{\hbar}\right) \psi_{N, t}\right\rangle \\
& +2 \mathrm{i} \hbar^{2} \operatorname{Im} \sum_{j=1}^{k}\left\langle\Delta_{q_{j}} a\left(f_{q_{k}, p_{k}}^{\hbar}\right) \cdots a\left(f_{q_{1}, p_{1}}^{\hbar}\right) \psi_{N, t}, a\left(f_{q_{k}, p_{k}}^{\hbar}\right) \cdots a\left(f_{q_{1}, p_{1}}^{\hbar}\right) \psi_{N, t}\right\rangle .
\end{aligned}
$$

Therefore it follows that

$$
\begin{aligned}
I_{2}= & -2 \mathrm{i} \hbar \mathbf{p}_{k} \cdot \nabla_{\mathbf{q}_{k}} m_{N, t}^{(k)}\left(q_{1}, p_{1}, \ldots, q_{k}, p_{k}\right) \\
& +2 \mathrm{i} \hbar^{2} \operatorname{Im} \sum_{j=1}^{k}\left\langle\Delta_{q_{j}} a\left(f_{q_{k}, p_{k}}^{\hbar}\right) \cdots a\left(f_{q_{1}, p_{1}}^{\hbar}\right) \psi_{N, t}, a\left(f_{q_{k}, p_{k}}^{\hbar}\right) \cdots a\left(f_{q_{1}, p_{1}}^{\hbar}\right) \psi_{N, t}\right\rangle .
\end{aligned}
$$

Now, we turn our focus on part $I_{2}$ of (3.14),

$$
\begin{aligned}
& I_{2} \\
& \left.=\frac{1}{N} \int \cdots \int(\mathrm{d} w \mathrm{~d} u)^{\otimes k}\left(f_{q, p}^{\hbar}(w) \overline{f_{q, p}^{\hbar}(u)}\right)\right)^{\otimes k} \iint \mathrm{d} x \mathrm{~d} y V(x-y) \\
& \quad\left\langle\psi, a_{w_{1}}^{*} \cdots a_{w_{k}}^{*} a_{u_{k}} \cdots a_{u_{1}} a_{x}^{*} a_{y}^{*} a_{y} a_{x} \psi\right\rangle \\
& \quad-\frac{1}{N} \int \cdots \int(\mathrm{d} w \mathrm{~d} u)^{\otimes k}\left(f_{q, p}^{\hbar}(w) \overline{f_{q, p}^{\hbar}(u)}\right)^{\otimes k} \iint \mathrm{d} x \mathrm{~d} y V(x-y) \\
& \\
& \quad\left\langle\psi, a_{x}^{*} a_{y}^{*} a_{y} a_{x} a_{w_{1}}^{*} \cdots a_{w_{k}}^{*} a_{u_{k}} \cdots a_{u_{1}} \psi\right\rangle .
\end{aligned}
$$


For $1 \leq k \leq N$, observe that from the CAR, we have

$$
\begin{aligned}
& a_{w_{1}}^{*} \cdots a_{w_{k}}^{*} a_{u_{k}} \cdots a_{u_{1}} a_{x}^{*} a_{y}^{*} a_{y} a_{x}-(-1)^{8 k} a_{x}^{*} a_{y}^{*} a_{y} a_{x} a_{w_{1}}^{*} \cdots a_{w_{k}}^{*} a_{u_{k}} \cdots a_{u_{1}} \\
& =-a_{w_{1}}^{*} \cdots a_{w_{k}}^{*}\left(\sum_{j=1}^{k}(-1)^{j} \delta_{x=u_{j}} a_{u_{k}} \cdots \widehat{a_{u_{j}}} \cdots a_{u_{1}}\right) a_{y}^{*} a_{y} a_{x} \\
& \quad-a_{x}^{*} a_{w_{1}}^{*} \cdots a_{w_{k}}^{*}\left(\sum_{j=1}^{k}(-1)^{j} \delta_{y=u_{j}} a_{u_{k}} \cdots \widehat{a_{u_{j}}} \cdots a_{u_{1}}\right) a_{y} a_{x} \\
& \quad+a_{x}^{*} a_{y}^{*}\left(\sum_{j=1}^{k}(-1)^{j} \delta_{y=w_{j}} a_{w_{1}}^{*} \cdots \widehat{a_{w_{j}}^{*}} \cdots a_{w_{k}}^{*}\right) a_{u_{k}} \cdots a_{u_{1}} a_{x} \\
& \quad+a_{x}^{*} a_{y}^{*} a_{y}\left(\sum_{j=1}^{k}(-1)^{j} \delta_{x=w_{j}} a_{w_{1}}^{*} \cdots \widehat{a_{w_{j}}^{*}} \cdots a_{w_{k}}^{*}\right) a_{u_{k}} \cdots a_{u_{1}}
\end{aligned}
$$

From (3.21), we have that

$$
\begin{aligned}
& \iint \mathrm{d} x \mathrm{~d} y V(x-y)\left(a_{w_{1}}^{*} \cdots a_{w_{k}}^{*} a_{u_{k}} \cdots a_{u_{1}} a_{x}^{*} a_{y}^{*} a_{y} a_{x}-a_{x}^{*} a_{y}^{*} a_{y} a_{x} a_{w_{1}}^{*} \cdots a_{w_{k}}^{*} a_{u_{k}} \cdots a_{u_{1}}\right) \\
& =\iint \mathrm{d} x \mathrm{~d} y V(x-y)\left[-a_{w_{1}}^{*} \cdots a_{w_{k}}^{*}\left(\sum_{j=1}^{k}(-1)^{j} \delta_{x=u_{j}} a_{u_{k}} \cdots \widehat{a_{u_{j}}} \cdots a_{u_{1}}\right) a_{y}^{*} a_{y} a_{x}\right. \\
& \quad-a_{x}^{*} a_{w_{1}}^{*} \cdots a_{w_{k}}^{*}\left(\sum_{j=1}^{k}(-1)^{j} \delta_{y=u_{j}} a_{u_{k}} \cdots \widehat{a_{u_{j}}} \cdots a_{u_{1}}\right) a_{y} a_{x} \\
& \quad+a_{x}^{*} a_{y}^{*} a_{y}\left(\sum_{j=1}^{k}(-1)^{j} \delta_{x=w_{j}} a_{w_{1}}^{*} \cdots \widehat{a_{w_{j}}^{*}} \cdots a_{w_{k}}^{*}\right) a_{u_{k}} \cdots a_{u_{1}} \\
& \left.\quad+a_{x}^{*} a_{y}^{*} a_{y}\left(\sum_{j=1}^{k}(-1)^{j} \delta_{x=w_{j}} a_{w_{1}}^{*} \cdots \widehat{a_{w_{j}}^{*}} \cdots a_{w_{k}}^{*}\right) a_{u_{k}} \cdots a_{u_{1}}\right] \\
& =: J_{1}+J_{2}+J_{3}+J_{4} .
\end{aligned}
$$

Note that summing $J_{1}$ and $J_{4}$, we have

$$
\begin{aligned}
J_{1}+J_{4}= & -\sum_{j=1}^{k}(-1)^{j} \int \mathrm{d} y\left[\left(V\left(u_{j}-y\right) a_{w_{1}}^{*} \cdots a_{w_{k}}^{*} a_{u_{k}} \cdots \widehat{a_{u_{j}}} \cdots a_{u_{1}} a_{y}^{*} a_{y} a_{u_{j}}\right)\right. \\
& \left.-\left(V\left(w_{j}-y\right) a_{w_{j}}^{*} a_{y}^{*} a_{y} a_{w_{1}}^{*} \cdots \widehat{a_{w_{j}}^{*}} \cdots a_{w_{k}}^{*} a_{u_{k}} \cdots a_{u_{1}}\right)\right] \\
= & \sum_{j=1}^{k}\left[\int \mathrm{d} y V\left(u_{j}-y\right) a_{w_{1}}^{*} \cdots a_{w_{k}}^{*} a_{u_{k}} \cdots a_{u_{1}} a_{y}^{*} a_{y}-V(0) a_{w_{1}}^{*} \cdots a_{w_{k}}^{*} a_{u_{k}} \cdots a_{u_{1}}\right] \\
& -\sum_{j=1}^{k}\left[\int \mathrm{d} y V\left(w_{j}-y\right) a_{y}^{*} a_{y} a_{w_{1}}^{*} \cdots a_{w_{k}}^{*} a_{u_{k}} \cdots a_{u_{1}}-V(0) a_{w_{1}}^{*} \cdots a_{w_{k}}^{*} a_{u_{k}} \cdots a_{u_{1}}\right],
\end{aligned}
$$


where the terms with $V(0)$ cancel one another. For the remaining term, we use again CAR to obtain

$$
\begin{aligned}
= & \sum_{j=1}^{k} \int \mathrm{d} y\left(V\left(u_{j}-y\right)-V\left(w_{j}-y\right)\right) a_{y}^{*} a_{w_{1}}^{*} \cdots a_{w_{k}}^{*} a_{u_{k}} \cdots a_{u_{1}} a_{y} \\
& +\sum_{j=1}^{k} \sum_{i=1}^{k}(-1)^{i} \int \mathrm{d} y V\left(u_{j}-y\right) \delta_{u_{i}=y} a_{w_{1}}^{*} \cdots a_{w_{k}}^{*} a_{u_{k}} \cdots \widehat{a_{u_{i}}} \cdots a_{u_{1}} a_{y} \\
& -\sum_{j=1}^{k} \sum_{i=1}^{k}(-1)^{i} \int \mathrm{d} y V\left(w_{j}-y\right) \delta_{w_{i}=y} a_{y}^{*} a_{w_{1}}^{*} \cdots \widehat{a_{w_{i}}^{*}} \cdots a_{w_{k}}^{*} a_{u_{k}} \cdots a_{u_{1}} \\
= & \sum_{j=1}^{k} \int \mathrm{d} y\left(V\left(u_{j}-y\right)-V\left(w_{j}-y\right)\right) a_{y}^{*} a_{w_{1}}^{*} \cdots a_{w_{k}}^{*} a_{u_{k}} \cdots a_{u_{1}} a_{y} \\
& -\sum_{j=1}^{k} \sum_{i=1}^{k}\left(V\left(u_{j}-u_{i}\right)-V\left(w_{j}-w_{i}\right)\right) a_{w_{1}}^{*} \cdots a_{w_{k}}^{*} a_{u_{k}} \cdots a_{u_{1}} .
\end{aligned}
$$

On the other hand, the sum of $J_{2}$ and $J_{2}$ yield

$$
J_{2}+J_{3}=\sum_{j=1}^{k} \int \mathrm{d} x\left(V\left(x-u_{j}\right)-V\left(x-w_{j}\right)\right) a_{x}^{*} a_{w_{1}}^{*} \cdots a_{w_{k}}^{*} a_{u_{k}} \cdots a_{u_{1}} a_{x} .
$$

By change of variable and using the fact that $V(-x)=V(x)$, we have from (3.21) that

$$
\begin{aligned}
I I_{2}= & \frac{2}{N} \int \cdots \int(\mathrm{d} w \mathrm{~d} u)^{\otimes k} \int \mathrm{d} y \sum_{j=1}^{k}\left[V\left(y-u_{j}\right)-V\left(w_{j}-y\right)\right]\left(f_{q, p}^{\hbar}(w) \overline{f_{q, p}^{\hbar}(u)}\right)^{\otimes k} \\
& \cdot\left\langle a_{w_{k}} \cdots a_{w_{1}} a_{y} \psi_{N, t}, a_{u_{k}} \cdots a_{u_{1}} a_{y} \psi_{N, t}\right\rangle \\
- & \frac{1}{N} \int \cdots \int(\mathrm{d} w \mathrm{~d} u)^{\otimes k} \sum_{j \neq i}^{k}\left[V\left(u_{j}-u_{i}\right)-V\left(w_{j}-w_{i}\right)\right]\left(f_{q, p}^{\hbar}(w) \overline{f_{q, p}^{\hbar}(u)}\right)^{\otimes k} \\
& \cdot\left\langle a_{w_{k}} \cdots a_{w_{1}} \psi_{N, t}, a_{u_{k}} \cdots a_{u_{1}} \psi_{N, t}\right\rangle
\end{aligned}
$$

Applying mean value theorem on the first term on right hand side, we have that

$$
\begin{aligned}
\frac{2}{N} \sum_{j=1}^{k} \int \cdots \int(\mathrm{d} w \mathrm{~d} u)^{\otimes k} \int \mathrm{d} y\left(V\left(y-u_{j}\right)-V\left(w_{j}-y\right)\right)\left(f_{q, p}^{\hbar}(w) \overline{f_{q, p}^{\hbar}(u)}\right)^{\otimes k} \\
\cdot\left\langle a_{w_{k}} \cdots a_{w_{1}} a_{y} \psi_{N, t}, a_{u_{k}} \cdots a_{u_{1}} a_{y} \psi_{N, t}\right\rangle \\
=\frac{2}{N} \sum_{j=1}^{k} \int \cdots \int(\mathrm{d} w \mathrm{~d} u)^{\otimes k} \int \mathrm{d} y\left[\int_{0}^{1} \mathrm{~d} s \nabla V\left(s u_{j}+(1-s) w_{j}-y\right)\right] \\
\cdot\left(u_{j}-w_{j}\right)\left(f_{q, p}^{\hbar}(w) \overline{f_{q, p}^{\hbar}(u)}\right)^{\otimes k} \\
\cdot\left\langle a_{w_{k}} \cdots a_{w_{1}} a_{y} \psi_{N, t}, a_{u_{k}} \cdots a_{u_{1}} a_{y} \psi_{N, t}\right\rangle \\
=\frac{2 \mathrm{i} \hbar}{N} \sum_{j=1}^{k} \int \cdots \int(\mathrm{d} w \mathrm{~d} u)^{\otimes k} \int \mathrm{d} y\left[\int_{0}^{1} \mathrm{~d} s \nabla V\left(s u_{j}+(1-s) w_{j}-y\right)\right]
\end{aligned}
$$




$$
\begin{aligned}
& \cdot \nabla_{p_{j}}\left(f_{q, p}^{\hbar}(w) \overline{f_{q, p}^{\hbar}(u)}\right)^{\otimes k} \\
& \cdot\left\langle a_{w_{k}} \cdots a_{w_{1}} a_{y} \psi_{N, t}, a_{u_{k}} \cdots a_{u_{1}} a_{y} \psi_{N, t}\right\rangle .
\end{aligned}
$$

As in the case of $k=1$, we apply the projection (3.11) onto $a_{y} \psi_{N, t}$ and get further

$$
\begin{aligned}
& \frac{2 \mathrm{i} \hbar}{N} \sum_{j=1}^{k} \int \cdots \int(\mathrm{d} w \mathrm{~d} u)^{\otimes k} \int \mathrm{d} y\left[\int_{0}^{1} \mathrm{~d} s \nabla V\left(s u_{j}+(1-s) w_{j}-y\right)\right] \cdot \nabla_{p_{j}}\left(f_{q, p}^{\hbar}(w) \overline{f_{q, p}^{\hbar}(u)}\right)^{\otimes k} \\
& \quad \cdot\left\langle a_{w_{k}} \cdots a_{w_{1}} a_{y} \psi_{N, t}, a_{u_{k}} \cdots a_{u_{1}} \mathbb{1} a_{y} \psi_{N, t}\right\rangle \\
& =\frac{2 \mathrm{i} \hbar}{N} \frac{1}{(2 \pi \hbar)^{3}} \sum_{j=1}^{k} \int \cdots \int(\mathrm{d} w \mathrm{~d} u)^{\otimes k} \int \mathrm{d} y\left[\int_{0}^{1} \mathrm{~d} s \nabla V\left(s u_{j}+(1-s) w_{j}-y\right)\right] \\
& \quad \cdot \nabla_{p_{j}}\left(f_{q, p}^{\hbar}(w) \overline{f_{q, p}^{\hbar}(u)}\right) \\
& \quad \cdot \iint \mathrm{d} \widetilde{q} d \widetilde{p} f_{\widetilde{q}, \widetilde{p}}^{\hbar}(y) \int \mathrm{d} v \overline{f_{\widetilde{q}, \widetilde{p}}^{\hbar}(v)}\left\langle a_{w_{k}} \cdots a_{w_{1}} a_{y} \psi_{N, t}, a_{u_{k}} \cdots a_{u_{1}} a_{v} \psi_{N, t}\right\rangle .
\end{aligned}
$$

Therefore, dividing both equations by $2 \mathrm{i} \hbar$, we have the following equation

$$
\begin{aligned}
& \partial_{t} m_{N, t}^{(k)}\left(q_{1}, p_{1}, \ldots, q_{k}, p_{k}\right)+\mathbf{p}_{k} \cdot \nabla_{\mathbf{q}_{k}} m_{N, t}^{(k)}\left(q_{1}, p_{1}, \ldots, q_{k}, p_{k}\right) \\
& =\hbar \operatorname{Im} \sum_{j=1}^{k}\left\langle\Delta_{q_{j}} a\left(f_{q_{k}, p_{k}}^{\hbar}\right) \cdots a\left(f_{q_{1}, p_{1}}^{\hbar}\right) \psi_{N, t}, a\left(f_{q_{k}, p_{k}}^{\hbar}\right) \cdots a\left(f_{q_{1}, p_{1}}^{\hbar}\right) \psi_{N, t}\right\rangle \\
& +\frac{1}{(2 \pi)^{3}} \sum_{j=1}^{k} \int \ldots \int(\mathrm{d} w \mathrm{~d} u)^{\otimes k} \int \mathrm{d} y\left[\int_{0}^{1} \mathrm{~d} s \nabla V\left(s u_{j}+(1-s) w_{j}-y\right)\right] \\
& \cdot \nabla_{p_{j}}\left(f_{q, p}^{\hbar}(w) \overline{f_{q, p}^{\hbar}(u)}\right)^{\otimes k} \\
& \cdot \iint \mathrm{d} \widetilde{q} d \widetilde{p} f_{\widetilde{q}, \widetilde{p}}^{\hbar}(y) \int \mathrm{d} v \overline{f_{\widetilde{q}, \widetilde{p}}^{\hbar}(v)}\left\langle a_{w_{k}} \cdots a_{w_{1}} a_{y} \psi_{N, t}, a_{u_{k}} \cdots a_{u_{1}} a_{v} \psi_{N, t}\right\rangle \\
& +\frac{\mathrm{i} \hbar^{2}}{2} \int \ldots \int(\mathrm{d} w \mathrm{~d} u)^{\otimes k} \sum_{j \neq i}^{k}\left[V\left(u_{j}-u_{i}\right)-V\left(w_{j}-w_{i}\right)\right]\left(f_{q, p}^{\hbar}(w) \overline{f_{q, p}^{\hbar}(u)}\right)^{\otimes k} \\
& \cdot\left\langle a_{w_{k}} \cdots a_{w_{1}} \psi_{N, t}, a_{u_{k}} \cdots a_{u_{1}} \psi_{N, t}\right\rangle \text {. }
\end{aligned}
$$

for $1 \leq k \leq N, \mathbf{p}_{k}=\left(p_{1}, \ldots, p_{k}\right)$ and recalling $\hbar^{3}=N^{-1}$. At this point we finish the computation of the hierarchy for Husimi measure.

\subsection{Proof of the Uniform Estimates in Section 2.3}

This subsection provide the proof of estimates for the error terms that appeared in the equations for $m_{N, t}^{(k)}$. Note that in all the proofs below, we suppose, without loss of generality, that the test function $\Phi \in C_{0}^{\infty}\left(\mathbb{R}^{6 k}\right)$ is factorized in phase-space by family of test functions in $C_{0}^{\infty}\left(\mathbb{R}^{3}\right)$ space. 


\subsubsection{Proof of Proposition 2.4}

Proof For fixed $k$, we denote the vector $\mathbf{x}_{k}=\left(x_{1}, \cdots, x_{k}\right)$ for each $x_{j} \in \mathbb{R}^{3}$ with $j=$ $1, \cdots, k$. Then we estimate the integral as follows

$$
\begin{aligned}
& \left|\int \cdots \int(\mathrm{d} q \mathrm{~d} p)^{\otimes k} \nabla_{\mathbf{q}_{k}} \Phi\left(q_{1}, p_{1}, \ldots, q_{k}, p_{k}\right) \cdot \mathcal{R}_{k}\right| \\
& \leq \hbar \mid \sum_{j=1}^{k} \int \cdots \int(\mathrm{d} q \mathrm{~d} p)^{\otimes k} \nabla_{q_{j}} \Phi\left(q_{1}, p_{1}, \ldots, q_{k}, p_{k}\right) \\
& \cdot\left\langle\nabla_{q_{j}}\left(a\left(f_{q_{k}, p_{k}}^{\hbar}\right) \cdots a\left(f_{q_{1}, p_{1}}^{\hbar}\right)\right) \psi_{N, t}, a\left(f_{q_{k}, p_{k}}^{\hbar}\right) \cdots a\left(f_{q_{1}, p_{1}}^{\hbar}\right) \psi_{N, t}\right\rangle \mid \\
& =\hbar^{1-\frac{3}{2} k} \mid \sum_{j=1}^{k} \int \ldots \int(\mathrm{d} q \mathrm{~d} p)^{\otimes k} \nabla_{q_{j}} \Phi\left(q_{1}, p_{1}, \ldots, q_{k}, p_{k}\right) \\
& \int \cdots \int(\mathrm{d} w \mathrm{~d} u)^{\otimes k} \prod_{n=1}^{k}\left(\chi_{\left(w_{n}-u_{n}\right) \in \Omega_{\hbar}^{c}}+\chi_{\left(w_{n}-u_{n}\right) \in \Omega_{\hbar}}\right) \\
& \cdot \nabla_{q_{j}} f\left(\frac{w_{n}-q_{n}}{\sqrt{\hbar}}\right) f\left(\frac{u_{n}-q_{n}}{\sqrt{\hbar}}\right) e^{\frac{i}{\hbar} p_{n} \cdot\left(w_{n}-u_{n}\right)}\left\langle a_{w_{k}} \cdots a_{w_{1}} \psi_{N, t}, a_{u_{k}} \cdots a_{u_{1}} \psi_{N, t}\right\rangle \mid \\
& \leq \hbar^{1-\frac{3}{2} k} \sum_{j=1}^{k} \int \cdots \int(\mathrm{d} q \mathrm{~d} w \mathrm{~d} u)^{\otimes k} \\
& \left|\int \cdots \int(\mathrm{d} p)^{\otimes k} \prod_{n=1}^{k}\left(\chi_{\left(w_{n}-u_{n}\right) \in \Omega_{\hbar}}+\chi_{\left(w_{n}-u_{n}\right) \in \Omega_{\hbar}^{c}}\right) \nabla_{q_{j}} \Phi \cdot e^{\frac{i}{\hbar} p_{n} \cdot\left(w_{n}-u_{n}\right)}\right| \\
& \text {. }\left|\nabla_{q_{j}} f\left(\frac{w_{n}-q_{n}}{\sqrt{\hbar}}\right)\right|\left|f\left(\frac{u_{n}-q_{n}}{\sqrt{\hbar}}\right)\right|\left\|a_{w_{k}} \cdots a_{w_{1}} \psi_{N, t}\right\|\left\|a_{u_{k}} \cdots a_{u_{1}} \psi_{N, t}\right\| \\
& =\hbar^{\frac{1}{2}-\frac{3}{2} k} \sum_{j=1}^{k} \int \cdots \int(\mathrm{d} q \mathrm{~d} w \mathrm{~d} u)^{\otimes k} \\
& \left|\int \cdots \int(\mathrm{d} p)^{\otimes k} \prod_{n=1}^{k}\left(\chi_{\left(w_{n}-u_{n}\right) \in \Omega_{\hbar}}+\chi_{\left(w_{n}-u_{n}\right) \in \Omega_{\hbar}^{c}}\right) \nabla_{q_{j}} \Phi \cdot e^{\frac{i}{\hbar} p_{n} \cdot\left(w_{n}-u_{n}\right)}\right| \\
& \cdot \prod_{n \neq j}^{k}\left|f\left(\frac{w_{n}-q_{n}}{\sqrt{\hbar}}\right) f\left(\frac{u_{n}-q_{n}}{\sqrt{\hbar}}\right)\right|\left|\nabla f\left(\frac{w_{j}-q_{j}}{\sqrt{\hbar}}\right)\right|\left|f\left(\frac{u_{j}-q_{j}}{\sqrt{\hbar}}\right)\right| \\
& \text { - }\left\|a_{w_{k}} \cdots a_{w_{1}} \psi_{N, t}\right\|\left\|a_{u_{k}} \cdots a_{u_{1}} \psi_{N, t}\right\|,
\end{aligned}
$$

where $\Omega_{\hbar}$ is defined as in (2.10) and used the fact that

$$
\nabla_{q_{j}} f\left(\frac{w_{j}-q_{j}}{\sqrt{\hbar}}\right)=-\frac{1}{\sqrt{\hbar}} \nabla f\left(\frac{w_{j}-q_{j}}{\sqrt{\hbar}}\right) .
$$

Now, the product term $\prod_{n=1}^{k}\left(\chi_{\left(w_{n}-u_{n}\right) \in \Omega_{\hbar}}+\chi_{\left(w_{n}-u_{n}\right) \in \Omega_{\hbar}^{c}}\right)$ in (3.27) includes a summation of $C(k)$ terms of the following type

$$
\chi_{\left(w_{1}-u_{1}\right) \in \Omega_{\hbar}} \cdots \chi_{\left(w_{\ell}-u_{\ell}\right) \in \Omega_{\hbar}} \chi_{\left(w_{\ell+1}-u_{\ell+1}\right) \in \Omega_{\hbar}^{c}} \cdots \chi_{\left(w_{k}-u_{k}\right) \in \Omega_{\hbar}^{c}},
$$


where $\ell \in\{1, \ldots, k\}$. Thus, to continue from (3.27), we have

$$
\begin{aligned}
& \left|\int \cdots \int(\mathrm{d} q \mathrm{~d} p)^{\otimes k} \nabla_{\mathbf{q}_{k}} \Phi\left(q_{1}, p_{1}, \ldots, q_{k}, p_{k}\right) \cdot \mathcal{R}_{k}\right| \\
& \leq C \hbar^{\frac{1}{2}-\frac{3}{2} k} \sum_{j=1}^{k} \max _{0 \leq \ell \leq k} \int \ldots \int(\mathrm{d} q \mathrm{~d} w \mathrm{~d} u)^{\otimes k} \prod_{n \neq j}^{k}\left|f\left(\frac{w_{n}-q_{n}}{\sqrt{\hbar}}\right) f\left(\frac{u_{n}-q_{n}}{\sqrt{\hbar}}\right)\right| \\
& \left|\nabla f\left(\frac{w_{j}-q_{j}}{\sqrt{\hbar}}\right)\right|\left|f\left(\frac{u_{j}-q_{j}}{\sqrt{\hbar}}\right)\right| \\
& \text {. } \mid \int \cdots \int(\mathrm{d} p)^{\otimes k}\left(\chi_{\left(w_{1}-u_{1}\right) \in \Omega_{\hbar}} \cdots \chi_{\left(w_{\ell}-u_{\ell}\right) \in \Omega_{\hbar}} \chi_{\left(w_{\ell+1}-u_{\ell+1}\right) \in \Omega_{\hbar}^{c}}\right. \\
& \left.\cdots \chi_{\left(w_{k}-u_{k}\right) \in \Omega_{\hbar}^{c}}\right) \nabla_{q_{j}} \Phi \cdot e^{\frac{i}{\hbar} \mathbf{p}_{k} \cdot\left(\mathbf{w}_{k}-\mathbf{u}_{k}\right)} \\
& \text { - }\left\|a_{w_{k}} \cdots a_{w_{1}} \psi_{N, t}\right\|\left\|a_{u_{k}} \cdots a_{u_{1}} \psi_{N, t}\right\| \\
& \leq C \hbar^{\frac{1}{2}-\frac{3}{2} k} \sum_{j=1}^{k} \max _{0 \leq \ell \leq k} \int \cdots \int(\mathrm{d} q \mathrm{~d} w \mathrm{~d} u)^{\otimes k} \prod_{n \neq j}^{k}\left|f\left(\frac{w_{n}-q_{n}}{\sqrt{\hbar}}\right) f\left(\frac{u_{n}-q_{n}}{\sqrt{\hbar}}\right)\right| \\
& \left|\nabla f\left(\frac{w_{j}-q_{j}}{\sqrt{\hbar}}\right)\right|\left|f\left(\frac{u_{j}-q_{j}}{\sqrt{\hbar}}\right)\right| \\
& \cdot \mid \int \ldots(\mathrm{d} p)^{\otimes \ell} \chi_{\left(w_{1}-u_{1}\right) \in \Omega_{\hbar}} \\
& \ldots \chi_{\left(w_{\ell}-u_{\ell}\right) \in \Omega_{\hbar}} e^{\frac{i}{\hbar} \sum_{m=1}^{\ell} p_{m} \cdot\left(w_{m}-u_{m}\right)} \\
& \cdot \int \cdots \int(\mathrm{d} p)^{\otimes(k-\ell)} \chi_{\left(w_{\ell+1}-u_{\ell+1}\right) \in \Omega_{\hbar}^{c}} \\
& \cdots \chi_{\left(w_{k}-u_{k}\right) \in \Omega_{\hbar}^{c}} e^{\frac{i}{\hbar} \sum_{m=k-\ell}^{\ell} p_{m} \cdot\left(w_{m}-u_{m}\right)} \nabla_{q_{j}} \Phi \\
& \text { - }\left\|a_{w_{k}} \cdots a_{w_{1}} \psi_{N, t}\right\|\left\|a_{u_{k}} \cdots a_{u_{1}} \psi_{N, t}\right\|
\end{aligned}
$$

Applying Lemma 2.5 onto the $(k-\ell)$ terms, we have

$$
\begin{aligned}
\leq C \sum_{j=1}^{k} \max _{0 \leq \ell \leq k} \hbar^{\frac{1}{2}-\frac{3}{2} k+(1-\alpha)(k-\ell) s} \int \ldots \int(\mathrm{d} q \mathrm{~d} w \mathrm{~d} u)^{\otimes k}\left(\chi_{\left(w_{1}-u_{1}\right) \in \Omega_{\hbar}} \cdots \chi_{\left(w_{\ell}-u_{\ell}\right) \in \Omega_{\hbar}}\right) \\
\cdot \prod_{n \neq j}^{k}\left|f\left(\frac{w_{n}-q_{n}}{\sqrt{\hbar}}\right) f\left(\frac{u_{n}-q_{n}}{\sqrt{\hbar}}\right)\right| \cdot\left|\nabla f\left(\frac{w_{j}-q_{j}}{\sqrt{\hbar}}\right)\right|\left|f\left(\frac{u_{j}-q_{j}}{\sqrt{\hbar}}\right)\right| \\
\cdot\left\|a_{w_{k}} \cdots a_{w_{1}} \psi_{N, t}\right\|\left\|a_{u_{k}} \cdots a_{u_{1}} \psi_{N, t}\right\| .
\end{aligned}
$$

For a fixed $\ell$, observe that since $f$ is compact supported, by using Hölder's inequality in $w$ and $u$ variables, we have

$$
\begin{gathered}
\int \cdots \int(\mathrm{d} q \mathrm{~d} w \mathrm{~d} u)^{\otimes k}\left(\chi_{\left(w_{1}-u_{1}\right) \in \Omega_{\hbar}} \cdots \chi_{\left(w_{\ell}-u_{\ell}\right) \in \Omega_{\hbar}}\right) \prod_{n \neq j}^{k}\left|f\left(\frac{w_{n}-q_{n}}{\sqrt{\hbar}}\right) f\left(\frac{u_{n}-q_{n}}{\sqrt{\hbar}}\right)\right| \\
\cdot\left|\nabla f\left(\frac{w_{j}-q_{j}}{\sqrt{\hbar}}\right)\right|\left|f\left(\frac{u_{j}-q_{j}}{\sqrt{\hbar}}\right)\right|\left\|a_{w_{k}} \cdots a_{w_{1}} \psi_{N, t}\right\|\left\|a_{u_{k}} \cdots a_{u_{1}} \psi_{N, t}\right\|
\end{gathered}
$$




$$
\begin{aligned}
= & \int \ldots \int(\mathrm{d} q \mathrm{~d} w \mathrm{~d} u)^{\otimes k}\left(\chi_{\left(w_{1}-u_{1}\right) \in \Omega_{\hbar}} \ldots \chi_{\left(w_{\ell}-u_{\ell}\right) \in \Omega_{\hbar}}\right) \prod_{n \neq j}^{k}\left|f\left(\frac{w_{n}-q_{n}}{\sqrt{\hbar}}\right) f\left(\frac{u_{n}-q_{n}}{\sqrt{\hbar}}\right)\right| \\
& \cdot\left|\nabla f\left(\frac{w_{j}-q_{j}}{\sqrt{\hbar}}\right)\right|\left|f\left(\frac{u_{j}-q_{j}}{\sqrt{\hbar}}\right)\right| \prod_{m=1}^{k} \chi_{\left|w_{m}-q_{m}\right| \leq \sqrt{\hbar} R} \chi_{\left|u_{m}-q_{m}\right| \leq \sqrt{\hbar} R} \\
& \left\|a_{w_{k}} \cdots a_{w_{1}} \psi_{N, t}\right\| \| a_{u_{k}} \cdots a_{u_{1}} \psi_{N, t} \mid \\
\leq & \int \cdots /(\mathrm{d} q)^{\otimes k}\left[\int \ldots \int(\mathrm{d} w \mathrm{~d} u)^{\otimes k}\left(\chi_{\left(w_{1}-u_{1}\right) \in \Omega_{\hbar}} \cdots \chi_{\left(w_{\ell}-u_{\ell}\right) \in \Omega_{\hbar}}\right)\right. \\
& \prod_{n \neq j}^{k}\left|f\left(\frac{w_{n}-q_{n}}{\sqrt{\hbar}}\right) f\left(\frac{u_{n}-q_{n}}{\sqrt{\hbar}}\right)\right|^{2} \\
& \left.\cdot\left|\nabla f\left(\frac{w_{j}-q_{j}}{\sqrt{\hbar}}\right)\right|^{2}\left|f\left(\frac{u_{j}-q_{j}}{\sqrt{\hbar}}\right)\right|^{2}\right]^{\frac{1}{2}} \\
& {\left[\int \cdots \int(\mathrm{d} w)^{\otimes k} \prod_{m=1}^{k} \chi_{\left|w_{m}-q_{m}\right| \leq \sqrt{\hbar} R}\left\|a_{w_{k}} \cdots a_{w_{1}} \psi_{N, t}\right\|^{2}\right] . }
\end{aligned}
$$

By change of variables and then applying Lemma 2.4, we have

$$
\begin{aligned}
= & \left.\hbar^{\frac{3}{2} k}\left[\int \cdots \int(\mathrm{d} \widetilde{w} \mathrm{~d} \widetilde{u})^{\otimes k}\left(\chi_{\left|\widetilde{w}_{1}-\widetilde{u}_{1}\right| \leq \hbar^{\alpha+\frac{1}{2}}} \cdots \chi_{\left|\widetilde{w}_{\ell}-\widetilde{u}_{\ell}\right| \leq \hbar^{\alpha+\frac{1}{2}}}\right) \prod_{n \neq j}^{k} \mid f\left(\widetilde{w}_{n}\right)\right) f\left(\widetilde{u}_{n}\right)\right|^{2} \\
& \left.\cdot\left|\nabla f\left(\widetilde{w}_{j}\right)\right|^{2}\left|f\left(\widetilde{u}_{j}\right)\right|^{2}\right]^{\frac{1}{2}} \int \cdots \int(\mathrm{d} q \mathrm{~d} w)^{\otimes k} \prod_{m=1}^{k} \chi_{\left|w_{m}-q_{m}\right| \leq \sqrt{\hbar} R}|| a_{w_{k}} \cdots a_{w_{1}} \psi_{N, t} \|^{2} \\
\leq & {\left[\int \cdots \int(\mathrm{d} \widetilde{w} \mathrm{~d} \tilde{u})^{\otimes k}\left(\chi_{\left|\widetilde{w}_{1}-\widetilde{u}_{1}\right| \leq \hbar^{\alpha+\frac{1}{2}}} \cdots \chi_{\left|\widetilde{w}_{\ell}-\widetilde{u}_{\ell}\right| \leq \hbar^{\alpha+\frac{1}{2}}}\right)\right.} \\
& \left.\left.\prod_{n \neq j}^{k} \mid f\left(\widetilde{w}_{n}\right)\right)\left.f\left(\widetilde{u}_{n}\right)\right|^{2}\left|\nabla f\left(\widetilde{w}_{j}\right)\right|^{2}\left|f\left(\widetilde{u}_{j}\right)\right|^{2}\right]^{\frac{1}{2}} .
\end{aligned}
$$

Observe now that by using Hölder inequality with respect to $\tilde{u}$, we get, for every $1 \leq n \leq k$,

$$
\begin{aligned}
& \int \mathrm{d} \widetilde{w}_{n}\left|f\left(\widetilde{w}_{n}\right)\right|^{2} \int \mathrm{d} \widetilde{u}_{n} \chi_{\left|\widetilde{w}_{n}-\widetilde{u}_{n}\right| \leq \hbar^{\alpha+\frac{1}{2}}}\left|f\left(\widetilde{u}_{n}\right)\right|^{2} \\
& \leq \int \mathrm{d} \widetilde{w}_{n}\left|f\left(\widetilde{w}_{n}\right)\right|^{2}\left(\int \mathrm{d} \widetilde{u}_{n} \chi_{\left|\widetilde{w}_{n}-\widetilde{u}_{n}\right| \leq \hbar^{\alpha+\frac{1}{2}}}\right)^{\frac{2}{3}}\left(\int \mathrm{d} \widetilde{u}_{n}\left|f\left(\widetilde{u}_{n}\right)\right|^{6}\right)^{\frac{1}{3}} \\
& \leq C \hbar^{2 \alpha+1}\left(\int \mathrm{d} \widetilde{w}_{n}\left|f\left(\widetilde{w}_{n}\right)\right|^{2}\right)\left(\int \mathrm{d} \widetilde{u}_{n}\left|f\left(\widetilde{u}_{n}\right)\right|^{6}\right)^{\frac{1}{3}} \\
& \leq C \hbar^{2 \alpha+1},
\end{aligned}
$$

where we have used the fact that $f \in H^{1}$, it is also embedded in the $L^{6}$ space. Similarly,

$$
\begin{aligned}
& \iint \mathrm{d} \widetilde{w}_{j} \mathrm{~d} \widetilde{u}_{j} \chi_{\left|\widetilde{w}_{j}-\tilde{u}_{j}\right| \leq \hbar^{\alpha+\frac{1}{2}}}\left|\nabla f\left(\widetilde{w}_{j}\right)\right|^{2}\left|f\left(\tilde{u}_{j}\right)\right|^{2} \\
& =\int \mathrm{d} \widetilde{w}_{j}\left|\nabla f\left(\widetilde{w}_{j}\right)\right|^{2} \int \mathrm{d} \tilde{u}_{j} \chi_{\left|\tilde{w}_{j}-\tilde{u}_{j}\right| \leq \hbar^{\alpha+\frac{1}{2}}}\left|f\left(\tilde{u}_{j}\right)\right|^{2}
\end{aligned}
$$




$$
\begin{aligned}
& \leq \int \mathrm{d} \tilde{w}_{j}\left|\nabla f\left(\tilde{w}_{j}\right)\right|^{2}\left(\int \mathrm{d} \tilde{u}_{j} \chi_{\left|\tilde{w}_{j}-\tilde{u}_{j}\right| \leq \hbar^{\alpha+\frac{1}{2}}}\right)^{\frac{2}{3}}\left(\int \mathrm{d} \tilde{u}_{j}\left|f\left(\tilde{u}_{j}\right)\right|^{6}\right)^{\frac{1}{3}} \\
& \leq C \hbar^{2 \alpha+1}
\end{aligned}
$$

Putting this back into (3.29), we have

$$
\begin{aligned}
& \int \ldots \int(\mathrm{d} q \mathrm{~d} w \mathrm{~d} u)^{\otimes k}\left(\chi_{\left(w_{1}-u_{1}\right) \in \Omega_{\hbar}} \cdots \chi_{\left(w_{\ell}-u_{\ell}\right) \in \Omega_{\hbar}}\right) \prod_{n \neq j}^{k}\left|f\left(\frac{w_{n}-q_{n}}{\sqrt{\hbar}}\right) f\left(\frac{u_{n}-q_{n}}{\sqrt{\hbar}}\right)\right| \\
& \quad \cdot\left|\nabla f\left(\frac{w_{j}-q_{j}}{\sqrt{\hbar}}\right)\right|\left|f\left(\frac{u_{j}-q_{j}}{\sqrt{\hbar}}\right)\right|\left\|a_{w_{k}} \cdots a_{w_{1}} \psi_{N, t}\right\|\left\|a_{u_{k}} \cdots a_{u_{1}} \psi_{N, t}\right\| \\
& \leq C \hbar^{\left(\alpha+\frac{1}{2}\right) \ell} .
\end{aligned}
$$

Then, from (3.28), we have

$$
\begin{aligned}
\left|\int \ldots \int(\mathrm{d} q \mathrm{~d} p)^{\otimes k} \nabla_{\mathbf{q}_{k}} \Phi\left(q_{1}, p_{1}, \ldots, q_{k}, p_{k}\right) \cdot \mathcal{R}_{k}\right| & \leq C \sum_{j=1}^{k} \max _{0 \leq \ell \leq k} \hbar^{\frac{1}{2}-\frac{3}{2} k+(1-\alpha)(k-\ell) s+\left(\alpha+\frac{1}{2}\right) \ell} \\
& =C k \max _{0 \leq \ell \leq k} \hbar^{\frac{1}{2}-\frac{3}{2} k+(1-\alpha)(k-\ell) s+\left(\alpha+\frac{1}{2}\right) \ell} .
\end{aligned}
$$

Therefore, by picking $s=\left\lceil\frac{1+2 \alpha}{2(1-\alpha)}\right\rceil$ we arrive immediately that

$$
\left|\int \cdots \int(\mathrm{d} q \mathrm{~d} p)^{\otimes k} \nabla_{\mathbf{q}_{k}} \Phi\left(q_{1}, p_{1}, \ldots, q_{k}, p_{k}\right) \cdot \mathcal{R}_{k}\right| \leq C \hbar^{\frac{1}{2}+(\alpha-1) k} .
$$

Therefore, for all $\delta \ll 1$, we choose $\frac{1}{2}<\alpha<1$ such that $(\alpha-1) k \leq-\delta$.

\subsubsection{Proof of Proposition 2.5}

Proof Let $\Phi$ be an arbitrary test function, then the remainder term $\widetilde{\mathcal{R}}_{1}$ can be written explicitly into

$$
\begin{aligned}
\mid \int & \mathrm{d} q_{1} \mathrm{~d} p_{1} \nabla_{p_{1}} \Phi\left(q_{1}, p_{1}\right) \cdot \widetilde{\mathcal{R}}_{1} \mid \\
= & \mid \iint \mathrm{d} q_{1} \mathrm{~d} p_{1} \nabla_{p_{1}} \Phi\left(q_{1}, p_{1}\right) \cdot\left(\iint \mathrm{d} w \mathrm{~d} u \iint \mathrm{d} y \mathrm{~d} v \iint \mathrm{d} q_{2} \mathrm{~d} p_{2}\right. \\
& \cdot\left[\int_{0}^{1} \mathrm{~d} s \nabla V(s u+(1-s) w-y)-\nabla V\left(q_{1}-q_{2}\right)\right] \\
& \left.\cdot f_{q_{1}, p_{1}}^{\hbar}(w) \overline{f_{q_{1}, p_{1}}^{\hbar}(u)} f_{q_{2}, p_{2}}^{\hbar}(y) \overline{f_{q_{2}, p_{2}}^{\hbar}(v)}\left\langle a_{w} a_{y} \psi_{N, t}, a_{u} a_{v} \psi_{N, t}\right\rangle\right) \mid \\
= & \frac{1}{\hbar^{3}} \mid \iint \mathrm{d} q_{1} \mathrm{~d} p_{1} \nabla_{p_{1}} \Phi\left(q_{1}, p_{1}\right) \cdot \iint \mathrm{d} w \mathrm{~d} u \iint \mathrm{d} y \mathrm{~d} v \iint \mathrm{d} q_{2} \mathrm{~d} p_{2} \\
& \cdot\left[\int_{0}^{1} \mathrm{~d} s \nabla V(s u+(1-s) w-y)-\nabla V\left(q_{1}-q_{2}\right)\right] e^{\frac{1}{\hbar} p_{1} \cdot(w-u)} e^{\frac{1}{\hbar} p_{2} \cdot(y-v)} \\
& \left.\cdot f\left(\frac{w-q_{1}}{\sqrt{\hbar}}\right) \overline{f\left(\frac{u-q_{1}}{\sqrt{\hbar}}\right)} f\left(\frac{y-q_{2}}{\sqrt{\hbar}}\right) \frac{v-q_{2}}{\sqrt{\hbar}}\right)\left\langle a_{w} a_{y} \psi_{N, t}, a_{u} a_{v} \psi_{N, t}\right\rangle .
\end{aligned}
$$


Then, utilizing (2.7), we may get

$$
\begin{aligned}
(2 \pi)^{3} \mid & \iint \mathrm{d} q_{1} \mathrm{~d} p_{1} \nabla_{p_{1}} \Phi\left(q_{1}, p_{1}\right) \cdot \iint \mathrm{d} w \mathrm{~d} u \iint \mathrm{d} y \mathrm{~d} q_{2} \\
\cdot & {\left[\int_{0}^{1} \mathrm{~d} s \nabla V(s u+(1-s) w-y)-\nabla V\left(q_{1}-q_{2}\right)\right] } \\
\cdot & f\left(\frac{w-q_{1}}{\sqrt{\hbar}}\right) \overline{f\left(\frac{u-q_{1}}{\sqrt{\hbar}}\right)} e^{\frac{\mathrm{i}}{\hbar} p_{1} \cdot(w-u)}\left|f\left(\frac{y-q_{2}}{\sqrt{\hbar}}\right)\right|^{2}\left\langle a_{w} a_{y} \psi_{N, t}, a_{u} a_{y} \psi_{N, t}\right\rangle \mid \\
= & (2 \pi)^{3} \hbar^{\frac{3}{2}} \mid \iint \mathrm{d} q_{1} \mathrm{~d} p_{1} \nabla_{p_{1}} \Phi\left(q_{1}, p_{1}\right) \cdot \iint \mathrm{d} w \mathrm{~d} u \iint \mathrm{d} y \mathrm{~d} \widetilde{q}_{2} \\
\cdot & {\left[\int_{0}^{1} \mathrm{~d} s \nabla V(s u+(1-s) w-y)-\nabla V\left(q_{1}-y+\sqrt{\hbar} \widetilde{q}_{2}\right)\right] } \\
\cdot & f\left(\frac{w-q_{1}}{\sqrt{\hbar}}\right) \overline{f\left(\frac{u-q_{1}}{\sqrt{\hbar}}\right)} e^{\frac{\mathrm{i}}{\hbar} p_{1} \cdot(w-u)}\left|f\left(\widetilde{q}_{2}\right)\right|^{2}\left\langle a_{w} a_{y} \psi_{N, t}, a_{u} a_{y} \psi_{N, t}\right\rangle \mid .
\end{aligned}
$$

Then, we insert a term, namely $\nabla V\left(q_{1}-y\right)$ and use triangle inequality to obtain

$$
\begin{aligned}
& \leq(2 \pi)^{3} \hbar^{\frac{3}{2}} \mid \iint \mathrm{d} q_{1} \mathrm{~d} p_{1} \nabla_{p_{1}} \Phi\left(q_{1}, p_{1}\right) \cdot \iint \mathrm{d} w \mathrm{~d} u \iint \mathrm{d} y \mathrm{~d} \widetilde{q}_{2} \\
& \quad \cdot \int_{0}^{1} \mathrm{~d} s\left(\nabla V(s u+(1-s) w-y)-\nabla V\left(q_{1}-y\right)\right) \\
& \quad \cdot f\left(\frac{w-q_{1}}{\sqrt{\hbar}}\right) \overline{f\left(\frac{u-q_{1}}{\sqrt{\hbar}}\right)} e^{\frac{\mathrm{i}}{\hbar} p_{1} \cdot(w-u)}\left|f\left(\widetilde{q}_{2}\right)\right|^{2}\left\langle a_{w} a_{y} \psi_{N, t}, a_{u} a_{y} \psi_{N, t}\right\rangle \mid \\
& \quad+(2 \pi)^{3} \hbar^{\frac{3}{2}} \mid \iint \mathrm{d} q_{1} \mathrm{~d} p_{1} \nabla_{p_{1}} \Phi\left(q_{1}, p_{1}\right) \\
& \quad \cdot \iint \mathrm{d} w \mathrm{~d} u \iint \mathrm{d} y \mathrm{~d} \widetilde{q}_{2}\left(\nabla V\left(q_{1}-y\right)-\nabla V\left(q_{1}-y+\sqrt{\hbar} \widetilde{q}_{2}\right)\right) \\
& \quad \cdot f\left(\frac{w-q_{1}}{\sqrt{\hbar}}\right) \overline{f\left(\frac{u-q_{1}}{\sqrt{\hbar}}\right)} e^{\frac{\mathrm{i}}{\hbar} p_{1} \cdot(w-u)}\left|f\left(\widetilde{q}_{2}\right)\right|^{2}\left\langle a_{w} a_{y} \psi_{N, t}, a_{u} a_{y} \psi_{N, t}\right\rangle \mid \\
& =: I_{3}+I I_{3},
\end{aligned}
$$

where we have used change of variable $\sqrt{\hbar} \widetilde{q}_{2}=\left(y-q_{2}\right)$ in the second term above.

We first focus on $I_{3}$. We begin by splitting the integral on momentum, by using Lemma 2.5 , it follows

$$
\begin{aligned}
I I_{3}= & (2 \pi)^{3} \hbar^{\frac{3}{2}} \mid \iint \mathrm{d} q_{1} \mathrm{~d} p_{1} \nabla_{p_{1}} \Phi\left(q_{1}, p_{1}\right) \cdot \iint \mathrm{d} w \mathrm{~d} u \iint \mathrm{d} y \mathrm{~d} \widetilde{q}_{2}\left(\chi_{(w-u) \in \Omega_{\hbar}^{c}}+\chi_{(w-u) \in \Omega_{\hbar}}\right) \\
& \cdot\left(\nabla V\left(q_{1}-y\right)-\nabla V\left(q_{1}-y+\sqrt{\hbar} \widetilde{q}_{2}\right)\right) f\left(\frac{w-q_{1}}{\sqrt{\hbar}}\right) \overline{f\left(\frac{u-q_{1}}{\sqrt{\hbar}}\right)} \\
& \cdot e^{\frac{1}{\hbar} p_{1} \cdot(w-u)}\left|f\left(\widetilde{q}_{2}\right)\right|^{2}\left\langle a_{w} a_{y} \psi_{N, t}, a_{u} a_{y} \psi_{N, t}\right\rangle \mid \\
\leq & (2 \pi)^{3} \hbar^{\frac{3}{2}+\frac{1}{2}} \int \mathrm{d} q_{1} \iint \mathrm{d} w \mathrm{~d} u \int \mathrm{d} y\left(\left|\int \mathrm{d} p_{1} e^{\frac{\mathrm{i}}{\hbar} p_{1} \cdot(w-u)} \chi_{(w-u) \in \Omega_{\hbar}^{c}} \nabla_{p_{1}} \Phi\left(q_{1}, p_{1}\right)\right|\right. \\
& \left.+\left|\int \mathrm{d} p_{1} e^{\frac{i}{\hbar} p_{1} \cdot(w-u)} \chi_{(w-u) \in \Omega_{\hbar}} \nabla_{p_{1}} \Phi\left(q_{1}, p_{1}\right)\right|\right) \cdot\left|f\left(\frac{w-q_{1}}{\sqrt{\hbar}}\right) f\left(\frac{u-q_{1}}{\sqrt{\hbar}}\right)\right|
\end{aligned}
$$




$$
\begin{aligned}
& \cdot\left(\int \mathrm{d} \widetilde{q}_{2}\left|\widetilde{q}_{2}\right|\left|f\left(\widetilde{q}_{2}\right)\right|^{2}\right)\left|\left\langle a_{w} a_{y} \psi_{N, t}, a_{u} a_{y} \psi_{N, t}\right\rangle\right| \\
\leq & C \hbar^{\frac{3}{2}+\frac{1}{2}} \int \mathrm{d} q_{1} \iint \mathrm{d} w \mathrm{~d} u \int \mathrm{d} y\left(\left|\int \mathrm{d} p_{1} e^{\frac{\mathrm{i}}{\hbar} p_{1} \cdot(w-u)} \chi_{(w-u) \in \Omega_{\hbar}^{c}} \nabla_{p_{1}} \Phi\left(q_{1}, p_{1}\right)\right|\right. \\
& \left.+\left|\int \mathrm{d} p_{1} e^{\frac{i}{\hbar} p_{1} \cdot(w-u)} \chi_{(w-u) \in \Omega_{\hbar}} \nabla_{p_{1}} \Phi\left(q_{1}, p_{1}\right)\right|\right) \cdot \mid f\left(\frac{w-q_{1}}{\sqrt{\hbar}}\right) \overline{f\left(\frac{u-q_{1}}{\sqrt{\hbar}}\right) \mid} \\
& \cdot\left|\left\langle a_{w} a_{y} \psi_{N, t}, a_{u} a_{y} \psi_{N, t}\right\rangle\right| \\
= & : i_{31}+i i_{31},
\end{aligned}
$$

where we used the fact that $\nabla V$ is Lipschitz continuous, $f$ has compact support, and the definition of $\Omega_{\hbar}$ in (2.10).

The next step is to use Lemmata 2.4 and 2.5 to bound the terms $i_{31}$ and $i i_{31}$. Then we examine what the appropriate terms $\alpha$ and $s$ should be. By Lemma 2.5, we may bound the term $i_{31}$, i.e.,

$$
\begin{aligned}
i_{31} & \leq C \hbar^{\frac{3}{2}+\frac{1}{2}+(1-\alpha) s} \int \mathrm{d} q_{1} \iint \mathrm{d} w \mathrm{~d} u \int \mathrm{d} y \cdot\left|f\left(\frac{w-q_{1}}{\sqrt{\hbar}}\right) \overline{f\left(\frac{u-q_{1}}{\sqrt{\hbar}}\right)}\right|\left|\left\langle a_{w} a_{y} \psi_{N, t}, a_{u} a_{y} \psi_{N, t}\right\rangle\right| \\
& \leq C \hbar^{\frac{3}{2}+\frac{1}{2}+(1-\alpha) s} \int \mathrm{d} q_{1} \iint \mathrm{d} w \mathrm{~d} u \int \mathrm{d} y\left|f\left(\frac{w-q_{1}}{\sqrt{\hbar}}\right) \overline{f\left(\frac{u-q_{1}}{\sqrt{\hbar}}\right)}\right|\left\|a_{w} a_{y} \psi_{N, t}\right\|\left\|a_{u} a_{y} \psi_{N, t}\right\| .
\end{aligned}
$$

Since we assume that $f$ is compactly supported, by Hölder inequality with respect to $w$ and $u$, we have we have that

$$
\begin{aligned}
i_{31} \leq C \hbar^{\frac{3}{2}+\frac{1}{2}+(1-\alpha) s} \int \mathrm{d} q_{1}\left(\iint \mathrm{d} w \mathrm{~d} u\left|f\left(\frac{w-q_{1}}{\sqrt{\hbar}}\right)\right|^{2}\left|f\left(\frac{u-q_{1}}{\sqrt{\hbar}}\right)\right|^{2}\right)^{\frac{1}{2}} \\
\cdot\left(\iint \mathrm{d} w \mathrm{~d} u \chi_{\left|w-q_{1}\right| \leq \sqrt{\hbar} R} \chi_{\left|u-q_{1}\right| \leq \sqrt{\hbar} R}\left(\int \mathrm{d} y\left\|a_{w} a_{y} \psi_{N, t}\right\|\left\|a_{u} a_{y} \psi_{N, t}\right\|\right)^{2}\right)^{\frac{1}{2}} \\
=C \hbar^{3+\frac{1}{2}+(1-\alpha) s} \int \mathrm{d} q_{1}\left(\int \mathrm{d} \widetilde{w} \mid f\left(\left.\widetilde{w}\right|^{2}\right)\right. \\
\quad\left(\iint \mathrm{d} w \mathrm{~d} u \chi_{\left|w-q_{1}\right| \leq \sqrt{\hbar} R} \chi_{\left|u-q_{1}\right| \leq \sqrt{\hbar} R}\left(\int \mathrm{d} y\left\|a_{w} a_{y} \psi_{N, t}\right\|\left\|a_{u} a_{y} \psi_{N, t}\right\|\right)^{2}\right)^{\frac{1}{2}},
\end{aligned}
$$

where we used the change of variable $\sqrt{\hbar} \widetilde{w}=w-q_{1}$ in the last inequality. Now, since $\|f\|_{2}$ is normalized, we continue to have

$$
\begin{aligned}
& \leq C \hbar^{3+\frac{1}{2}+(1-\alpha) s} \\
& \quad \int \mathrm{d} q_{1}\left(\iint \mathrm{d} w \mathrm{~d} u \chi_{\left|w-q_{1}\right| \leq \sqrt{\hbar} R} \chi_{\left|u-q_{1}\right| \leq \sqrt{\hbar} R}\left(\int \mathrm{d} y\left\|a_{w} a_{y} \psi_{N, t}\right\|\left\|a_{u} a_{y} \psi_{N, t}\right\|\right)^{2}\right)^{\frac{1}{2}} \\
& \leq C \hbar^{3+\frac{1}{2}+(1-\alpha) s} \\
& \quad \int \mathrm{d} q_{1}\left(\iint \mathrm{d} w \mathrm{~d} u \chi_{\left|w-q_{1}\right| \leq \sqrt{\hbar} R} \chi_{\left|u-q_{1}\right| \leq \sqrt{\hbar} R}\left(\int \mathrm{d} y\left\|a_{w} a_{y} \psi_{N, t}\right\|^{2}\right)\left(\int \mathrm{d} y\left\|a_{u} a_{y} \psi_{N, t}\right\|^{2}\right)\right)^{\frac{1}{2}}
\end{aligned}
$$




$$
\begin{aligned}
& =C \hbar^{3+\frac{1}{2}+(1-\alpha) s} \int \mathrm{d} q_{1} \iint \mathrm{d} y \mathrm{~d} w \chi_{\left|w-q_{1}\right| \leq \sqrt{\hbar} R}\left\|a_{w} a_{y} \psi_{N, t}\right\|^{2} \\
& =C \hbar^{3+\frac{1}{2}+(1-\alpha) s} \int \mathrm{d} y \iint \mathrm{d} q_{1} \mathrm{~d} w\left\langle a_{y} \psi_{N, t}, \chi_{\left|w-q_{1}\right| \leq \sqrt{\hbar} R} a_{w}^{*} a_{w} a_{y} \psi_{N, t}\right\rangle
\end{aligned}
$$

by Lemma 2.4

$$
\begin{aligned}
i_{31} & \leq C \hbar^{3-\frac{3}{2}+\frac{1}{2}+(1-\alpha) s} \int \mathrm{d} y\left\langle a_{y} \psi_{N, t}, a_{y} \psi_{N, t}\right\rangle \\
& =C \hbar^{(1-\alpha) s-1}\left\langle\psi_{N, t}, \frac{\mathcal{N}}{N} \psi_{N, t}\right\rangle \leq C \hbar^{(1-\alpha) s-1} .
\end{aligned}
$$

On the other hand, from $i i_{31}$ we have

$$
\begin{aligned}
& i i_{31} \leq C \hbar^{\frac{3}{2}+\frac{1}{2}} \int \mathrm{d} q_{1} \iint \mathrm{d} w \mathrm{~d} u \int \mathrm{d} y\left|\int \mathrm{d} p_{1} e^{\frac{\mathrm{i}}{\hbar} p_{1} \cdot(w-u)} \chi_{(w-u) \in \Omega_{\hbar}} \nabla_{p_{1}} \Phi\left(q_{1}, p_{1}\right)\right| \\
& \cdot\left|f\left(\frac{w-q_{1}}{\sqrt{\hbar}}\right) \overline{f\left(\frac{u-q_{1}}{\sqrt{\hbar}}\right)}\right|\left|\left\langle a_{w} a_{y} \psi_{N, t}, a_{u} a_{y} \psi_{N, t}\right\rangle\right| \\
& \leq C \hbar^{\frac{3}{2}+\frac{1}{2}} \int \mathrm{d} q_{1} \iint \mathrm{d} w \mathrm{~d} u \int \mathrm{d} y \int \mathrm{d} p_{1} \chi_{(w-u) \in \Omega_{\hbar}}\left|\nabla_{p_{1}} \Phi\left(q_{1}, p_{1}\right)\right| \\
& \cdot\left|f\left(\frac{w-q_{1}}{\sqrt{\hbar}}\right) \overline{f\left(\frac{u-q_{1}}{\sqrt{\hbar}}\right)}\right|\left|\left\langle a_{w} a_{y} \psi_{N, t}, a_{u} a_{y} \psi_{N, t}\right\rangle\right| \\
& \leq C \hbar^{\frac{3}{2}+\frac{1}{2}} \int \mathrm{d} q_{1} \iint \mathrm{d} w \mathrm{~d} u \int \mathrm{d} y \chi_{(w-u) \in \Omega_{\hbar}} \\
& \cdot\left|f\left(\frac{w-q_{1}}{\sqrt{\hbar}}\right) \overline{f\left(\frac{u-q_{1}}{\sqrt{\hbar}}\right)}\right|\left|\left\langle a_{w} a_{y} \psi_{N, t}, a_{u} a_{y} \psi_{N, t}\right\rangle\right|
\end{aligned}
$$

Since $f$ is assumed to be compactly supported, we have

$$
\begin{gathered}
\leq C \hbar^{\frac{3}{2}+\frac{1}{2}} \int \mathrm{d} q_{1}\left(\iint \mathrm{d} w \mathrm{~d} u \chi_{(w-u) \in \Omega_{\hbar}}\left|f\left(\frac{w-q_{1}}{\sqrt{\hbar}}\right) f\left(\frac{u-q_{1}}{\sqrt{\hbar}}\right)\right|^{2}\right)^{\frac{1}{2}} \\
\cdot\left(\iint \mathrm{d} w \mathrm{~d} u \chi_{\left|w-q_{1}\right| \leq \sqrt{\hbar} R} \chi_{\left|u-q_{1}\right| \leq \sqrt{\hbar} R}\left(\int \mathrm{d} y\left\|a_{w} a_{y} \psi_{N, t}\right\|\left\|a_{u} a_{y} \psi_{N, t}\right\|\right)^{2}\right)^{\frac{1}{2}},
\end{gathered}
$$

where we use Cauchy-Schwarz inequality and Hölder inequality.

Next, by change of variables as well as Hölder inequality in respect of $y$, we have

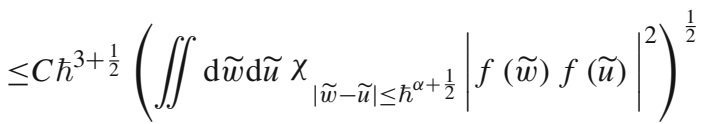

$$
\begin{aligned}
& \cdot \int \mathrm{d} y \iint \mathrm{d} q_{1} \mathrm{~d} w \chi_{\left|w-q_{1}\right| \leq \sqrt{\hbar} R}\left\langle a_{y} \psi_{N, t}, a_{w}^{*} a_{w} a_{y} \psi_{N, t}\right\rangle \\
& \leq C \hbar^{-1}\left(\int \mathrm{d} \tilde{w}|f(\widetilde{w})|^{2} \int \mathrm{d} \widetilde{u} \chi_{|\widetilde{w}-\widetilde{u}| \leq \hbar^{\alpha+\frac{1}{2}}}|f(\widetilde{u})|^{2}\right)^{\frac{1}{2}},
\end{aligned}
$$

where we applied Lemma 2.4. Observe from (3.30), we get

$$
i i_{31} \leq C \hbar^{\alpha-\frac{1}{2}} .
$$


Now we compare power of $\hbar$ with the one in (3.33). Namely,

$$
\alpha-\frac{1}{2}=(1-\alpha) s-1
$$

Therefore, we choose $s=\left\lceil\frac{1+2 \alpha}{2(1-\alpha)}\right\rceil$ such that $I_{3}$ is of order $\hbar^{\alpha-\frac{1}{2}}$. Now, focus on $I_{3}$, we use similar strategy as with $\mathrm{II}_{3}$.

$$
\begin{aligned}
I_{3} \leq & C \hbar^{\frac{3}{2}} \int \mathrm{d} q_{1} \iint \mathrm{d} w \mathrm{~d} u \int \mathrm{d} y \int_{0}^{1} \mathrm{~d} s\left|\nabla V(s u+(1-s) w-y)-\nabla V\left(q_{1}-y\right)\right| \\
& \cdot\left(\left|\int \mathrm{d} p_{1} e^{\frac{\mathrm{i}}{\hbar} p_{1} \cdot(w-u)} \chi_{(w-u) \in \Omega_{\hbar}^{c}} \nabla_{p_{1}} \Phi\left(q_{1}, p_{1}\right)\right|\right. \\
& \left.+\left|\int \mathrm{d} p_{1} e^{\frac{i}{\hbar} p_{1} \cdot(w-u)} \chi_{(w-u) \in \Omega_{\hbar}} \nabla_{p_{1}} \Phi\left(q_{1}, p_{1}\right)\right|\right) \\
& \cdot\left|f\left(\frac{w-q_{1}}{\sqrt{\hbar}}\right) \overline{f\left(\frac{u-q_{1}}{\sqrt{\hbar}}\right)}\right|\left(\int \mathrm{d} \widetilde{q}_{2}\left|f\left(\widetilde{q}_{2}\right)\right|^{2}\right)\left|\left\langle a_{w} a_{y} \psi_{N, t}, a_{u} a_{y} \psi_{N, t}\right\rangle\right| \\
\leq & C \hbar^{\frac{3}{2}} \int \mathrm{d} q_{1} \iint \mathrm{d} w \mathrm{~d} u \int \mathrm{d} y \\
& \int_{0}^{1} \mathrm{~d} s\left|s u+(1-s) w-q_{1}\right|\left(\left|\int \mathrm{d} p_{1} e^{\frac{\mathrm{i}}{\hbar} p_{1} \cdot(w-u)} \chi_{(w-u) \in \Omega_{\hbar}^{c} \nabla_{p_{1}}} \Phi\left(q_{1}, p_{1}\right)\right|\right. \\
& \left.+\left|\int \mathrm{d} p_{1} e^{\frac{\mathrm{i}}{\hbar} p_{1} \cdot(w-u)} \chi_{(w-u) \in \Omega_{\hbar}} \nabla_{p_{1}} \Phi\left(q_{1}, p_{1}\right)\right|\right) \cdot\left|f\left(\frac{w-q_{1}}{\sqrt{\hbar}}\right) f\left(\frac{u-q_{1}}{\sqrt{\hbar}}\right)\right| \\
& \cdot \chi_{\left|w-q_{1}\right| \leq \sqrt{\hbar} R} \chi_{\left|u-q_{1}\right| \leq \sqrt{\hbar} R}\left\|a_{w} a_{y} \psi_{N, t}\right\| \mid\left\|a_{u} a_{y} \psi_{N, t}\right\| \\
= & : i_{32}+i i_{32}
\end{aligned}
$$

Again, by Lemma 2.5 and the bounds for number operator and localized number operator, we have for $i_{32}$ that

$$
\begin{aligned}
& i_{32 \leq} \leq C \hbar^{\frac{3}{2}+(1-\alpha) s} \int \mathrm{d} q_{1} \iint \mathrm{d} w \mathrm{~d} u \int_{0}^{1} \mathrm{~d} s\left|s u+(1-s) w-q_{1}\right| \cdot \mid f\left(\frac{w-q_{1}}{\sqrt{\hbar}}\right) \overline{\left(\frac{u-q_{1}}{\sqrt{\hbar}}\right) \mid} \\
& \cdot \chi_{\left|w-q_{1}\right| \leq \sqrt{\hbar} R} \chi_{\left|u-q_{1}\right| \leq \sqrt{\hbar} R} \int \mathrm{d} y\left\|a_{w} a_{y} \psi_{N, t}\right\|\left\|a_{u} a_{y} \psi_{N, t}\right\| \\
& \leq C \hbar^{3+\frac{1}{2}+(1-\alpha) s} \int \mathrm{d} q_{1}\left(\iint \mathrm{d} \tilde{w} \mathrm{~d} \tilde{u} \int_{0}^{1} \mathrm{~d} s|s \tilde{u}+(1-s) \tilde{w}|^{2} \cdot|f(\widetilde{w}) f(\tilde{u})|^{2}\right)^{\frac{1}{2}} \\
& \quad\left(\iint \mathrm{d} w \mathrm{~d} u \chi_{\left|w-q_{1}\right| \leq \sqrt{\hbar} R} \chi_{\left|u-q_{1}\right| \leq \sqrt{\hbar} R}\left(\int \mathrm{d} y\left\|a_{w} a_{y} \psi_{N, t}\right\|\left\|a_{u} a_{y} \psi_{N, t}\right\|\right)^{2}\right)^{\frac{1}{2}} \\
& \leq C \hbar^{3+\frac{1}{2}+(1-\alpha) s}\left(\iint \mathrm{d} \tilde{w} \mathrm{~d} \tilde{u} \int_{0}^{1} \mathrm{~d} s|s \tilde{u}+(1-s) \tilde{w}|^{2} \cdot|f(\widetilde{w}) f(\widetilde{u})|^{2}\right)^{\frac{1}{2}} \\
& \quad \cdot \int \mathrm{d} y \iint \mathrm{d} q_{1} \mathrm{~d} w \chi_{\left|w-q_{1}\right| \leq \sqrt{\hbar} R}\left\langle a_{y} \psi_{N, t}, a_{w}^{*} a_{w} a_{y} \psi_{N, t}\right\rangle \\
& \leq C \hbar^{(1-\alpha) s-1},
\end{aligned}
$$


where we used Lemma 2.4 and the bounds for number operator. Similarly, for $i i_{32}$, we have

$$
\begin{aligned}
& i i_{32} \leq C \hbar^{\frac{3}{2}} \int \mathrm{d} q_{1} \iint \mathrm{d} w \mathrm{~d} u \int \mathrm{d} y \int_{0}^{1} \mathrm{~d} s\left|s u+(1-s) w-q_{1}\right| \int \mathrm{d} p_{1}\left|\chi_{(w-u) \in \Omega_{\hbar}} \nabla_{p_{1}} \Phi\left(q_{1}, p_{1}\right)\right| \\
& \quad \cdot \mid f\left(\frac{w-q_{1}}{\sqrt{\hbar}}\right) \overline{\left(\frac{u-q_{1}}{\sqrt{\hbar}}\right) \mid \chi_{\left|w-q_{1}\right| \leq \sqrt{\hbar} R} \chi_{\left|u-q_{1}\right| \leq \sqrt{\hbar} R}\left\|a_{w} a_{y} \psi_{N, t}\right\|\left\|a_{u} a_{y} \psi_{N, t}\right\|} \\
& \leq C \hbar^{\frac{3}{2}} \int \mathrm{d} q_{1} \iint \mathrm{d} w \mathrm{~d} u \int \mathrm{d} y \int_{0}^{1} \mathrm{~d} s\left|s u+(1-s) w-q_{1}\right| \chi_{(w-u) \in \Omega_{\hbar}}\left|f\left(\frac{w-q_{1}}{\sqrt{\hbar}}\right) f\left(\frac{u-q_{1}}{\sqrt{\hbar}}\right)\right| \\
& \quad \cdot \chi_{\left|w-q_{1}\right| \leq \sqrt{\hbar} R} \chi_{\left|u-q_{1}\right| \leq \sqrt{\hbar} R} \mid a_{w} a_{y} \psi_{N, t}\|\| a_{u} a_{y} \psi_{N, t} \| \\
& \leq C \hbar^{\frac{3}{2}} \int \mathrm{d} q_{1}\left(\iint \mathrm{d} w \mathrm{~d} u \int_{0}^{1} \mathrm{~d} s\left|s u+(1-s) w-q_{1}\right|^{2}\left|f\left(\frac{w-q_{1}}{\sqrt{\hbar}}\right) f\left(\frac{u-q_{1}}{\sqrt{\hbar}}\right)\right|^{2} \chi_{(w-u) \in \Omega_{\hbar}}\right)^{\frac{1}{2}} \\
& \quad \cdot\left(\int \mathrm{d} w \mathrm{~d} u \chi_{\left|w-q_{1}\right| \leq \sqrt{\hbar} R} \chi_{\left|u-q_{1}\right| \leq \sqrt{\hbar} R} \int \mathrm{d} y\left\|a_{w} a_{y} \psi_{N, t}\right\|\left\|a_{u} a_{y} \psi_{N, t}\right\|\right)^{\frac{1}{2}} \\
& \leq C \hbar^{3+\frac{1}{2}}\left(\iint \mathrm{d} \widetilde{w} \mathrm{~d} \tilde{u} \int_{0}^{1} \mathrm{~d} s|s \tilde{u}+(1-s) \widetilde{w}|^{2}|f(\widetilde{w}) f(\widetilde{u})|^{2} \chi_{|\widetilde{w}-\widetilde{u}| \leq \hbar^{\alpha+\frac{1}{2}}}\right)^{\frac{1}{2}} \\
& \quad \int \mathrm{d} q_{1}\left(\iint \mathrm{d} w \mathrm{~d} u \chi_{\left|w-q_{1}\right| \leq \sqrt{\hbar} R} \chi_{\left|u-q_{1}\right| \leq \sqrt{\hbar} R} \int \mathrm{d} y\left\|a_{w} a_{y} \psi_{N, t}\right\|\left\|a_{u} a_{y} \psi_{N, t}\right\|\right)^{\frac{1}{2}} .
\end{aligned}
$$

By Lemma 2.4 and the bounds for number operator, we have

$$
\leq C \hbar^{-1}\left(\iint \mathrm{d} \tilde{w} \mathrm{~d} \tilde{u} \int_{0}^{1} \mathrm{~d} s|s \tilde{u}+(1-s) \widetilde{w}|^{2}|f(\widetilde{w}) f(\widetilde{u})|^{2} \chi_{|\widetilde{w}-\widetilde{u}| \leq \hbar^{\alpha+\frac{1}{2}}}\right)^{\frac{1}{2}} .
$$

Then, by using similar computation in (3.30) and the assumption that $f$ is compactly supported, we may get

$$
i i_{32} \leq C \hbar^{\alpha-\frac{1}{2}}
$$

Therefore, $I_{3}$ and $I_{3}$ together, we have the bound of order $\hbar^{\alpha-\frac{1}{2}}$ for $\alpha \in\left(\frac{1}{2}, 1\right)$.

\subsubsection{Proof of Proposition 2.6}

Proof To calculate the bound in (2.21) for $\widehat{\mathcal{R}}_{k}$. It has automatically an $1 / N$ as a factor, therefore, we expect it has better estimates than the other remainder terms. More precisely, we can split the integrals as before,

$$
\begin{aligned}
& \mid \frac{1}{2 N} \int \ldots \int(\mathrm{d} q \mathrm{~d} p)^{\otimes k}(\mathrm{~d} w \mathrm{~d} u)^{\otimes k} \Phi\left(q_{1}, p_{1}, \ldots, q_{k}, p_{k}\right) \sum_{j \neq i}^{k}\left[V\left(u_{j}-u_{i}\right)-V\left(w_{j}-w_{i}\right)\right] \\
& \cdot\left(f_{q, p}^{\hbar}(w) \overline{f_{q, p}^{\hbar}(u)}\right)^{\otimes k}\left\langle a_{w_{k}} \cdots a_{w_{1}} \psi_{N, t}, a_{u_{k}} \cdots a_{u_{1}} \psi_{N, t}\right\rangle \mid \\
& =\mid \frac{1}{2 N} \int \cdots \int(\mathrm{d} q \mathrm{~d} p)^{\otimes k}(\mathrm{~d} w \mathrm{~d} u)^{\otimes k} \Phi\left(q_{1}, p_{1}, \ldots, q_{k}, p_{k}\right) \\
& \quad \sum_{j \neq i}^{k}\left[V\left(u_{j}-u_{i}\right)-V\left(w_{j}-w_{i}\right)\right]\left(f_{q, p}^{\hbar}(w) \overline{f_{q, p}^{\hbar}(u)}\right)^{\otimes k}
\end{aligned}
$$




$$
\cdot \prod_{n=1}^{k}\left(\chi_{\left(w_{n}-u_{n}\right) \in \Omega_{\hbar}^{c}}+\chi_{\left(w_{n}-u_{n}\right) \in \Omega_{\hbar}}\right)\left\langle a_{w_{k}} \cdots a_{w_{1}} \psi_{N, t}, a_{u_{k}} \cdots a_{u_{1}} \psi_{N, t}\right\rangle \mid,
$$

where $\Omega_{\hbar}$ is defined as in (2.10). Since $V \in W^{2, \infty}$ and recall $\hbar^{3}=N^{-1}$, we have

$$
\begin{aligned}
& \leq C(k)\|V\|_{\infty} \hbar^{3-\frac{3}{2} k} \int \ldots \int(\mathrm{d} q \mathrm{~d} w \mathrm{~d} u)^{\otimes k} \prod_{n=1}^{k}\left|f\left(\frac{w_{n}-q_{n}}{\sqrt{\hbar}}\right) f\left(\frac{u_{n}-q_{n}}{\sqrt{\hbar}}\right)\right| \\
& \left\|a_{w_{k}} \cdots a_{w_{1}} \psi_{N, t}\right\|\left\|a_{u_{k}} \cdots a_{u_{1}} \psi_{N, t}\right\| \\
& \cdot\left|\prod_{n=1}^{k}\left(\chi_{\left(w_{n}-u_{n}\right) \in \Omega_{\hbar}^{c}}+\chi_{\left(w_{n}-u_{n}\right) \in \Omega_{\hbar}}\right) \int \ldots \int(\mathrm{d} p)^{\otimes k} e^{\frac{i}{\hbar} \sum_{m=1}^{k} p_{m} \cdot\left(w_{m}-u_{m}\right)} \Phi\left(q_{1}, \ldots, p_{k}\right)\right| \\
& \leq C \hbar^{3-\frac{3}{2} k} \max _{0 \leq \ell \leq k} \int \ldots \int(\mathrm{d} q \mathrm{~d} w \mathrm{~d} u)^{\otimes k} \prod_{n=1}^{k}\left|f\left(\frac{w_{n}-q_{n}}{\sqrt{\hbar}}\right) f\left(\frac{u_{n}-q_{n}}{\sqrt{\hbar}}\right)\right| \\
& \left\|a_{w_{k}} \cdots a_{w_{1}} \psi_{N, t}\right\|\left\|a_{u_{k}} \cdots a_{u_{1}} \psi_{N, t}\right\| \cdot \mid \int \cdots \int(\mathrm{d} p)^{\otimes k} \\
& \left(\chi_{\left(w_{1}-u_{1}\right) \in \Omega_{\hbar}} \cdots \chi_{\left(w_{\ell}-u_{\ell}\right) \in \Omega_{\hbar}} \chi_{\left(w_{\ell+1}-u_{\ell+1}\right) \in \Omega_{\hbar}^{c}} \cdots \chi_{\left(w_{k}-u_{k}\right) \in \Omega_{\hbar}^{c}}\right) \\
& \nabla_{q_{j}} \Phi \cdot e^{\frac{i}{\hbar} \mathbf{p}_{k} \cdot\left(\mathbf{w}_{k}-\mathbf{u}_{k}\right)} \\
& =C \hbar^{3-\frac{3}{2} k} \max _{0 \leq \ell \leq k} \int \ldots \int(\mathrm{d} q \mathrm{~d} w \mathrm{~d} u)^{\otimes k} \mid \int \ldots \int(\mathrm{d} p)^{\otimes \ell} \chi_{\left(w_{1}-u_{1}\right) \in \Omega_{\hbar}} \cdots \chi_{\left(w_{\ell}-u_{\ell}\right) \in \Omega_{\hbar}} e^{\frac{i}{\hbar} \sum_{m=1}^{\ell} p_{m} \cdot\left(w_{m}-u_{m}\right)} \\
& \int \cdot \cdots(\mathrm{d} p)^{\otimes(k-\ell)} \chi_{\left(w_{\ell+1}-u_{\ell+1}\right) \in \Omega_{\hbar}^{c}} \\
& \ldots \chi_{\left(w_{k}-u_{k}\right) \in \Omega_{\hbar}^{c}} e^{\frac{i}{\hbar} \sum_{m=k-\ell}^{\ell} p_{m} \cdot\left(w_{m}-u_{m}\right)} \nabla_{q_{j}} \Phi\left(q_{1}, p_{1}, \ldots, q_{k}, p_{k}\right) \mid \\
& \cdot \prod_{n=1}^{k}\left|f\left(\frac{w_{n}-q_{n}}{\sqrt{\hbar}}\right) f\left(\frac{u_{n}-q_{n}}{\sqrt{\hbar}}\right)\right|\left\|a_{w_{k}} \cdots a_{w_{1}} \psi_{N, t}\right\|\left\|a_{u_{k}} \cdots a_{u_{1}} \psi_{N, t}\right\|,
\end{aligned}
$$

where we apply similar argument in (3.28) in the last inequality. Note here that the constant $C$ above is dependent on $k$. Applying Lemma 2.5 we have

$$
\begin{aligned}
\leq & C \max _{0 \leq \ell \leq k} \hbar^{3-\frac{3}{2} k+(1-\alpha)(k-\ell) s} \int \cdots /(\mathrm{d} q \mathrm{~d} w \mathrm{~d} u)^{\otimes k}\left(\chi_{\left(w_{1}-u_{1}\right) \in \Omega_{\hbar}} \cdots \chi_{\left(w_{\ell}-u_{\ell}\right) \in \Omega_{\hbar}}\right) \\
& \cdot \prod_{n=1}^{k}\left|f\left(\frac{w_{n}-q_{n}}{\sqrt{\hbar}}\right) f\left(\frac{u_{n}-q_{n}}{\sqrt{\hbar}}\right)\right|\left\|a_{w_{k}} \cdots a_{w_{1}} \psi_{N, t}\right\|\left\|a_{u_{k}} \cdots a_{u_{1}} \psi_{N, t}\right\| \\
= & C \max _{0 \leq \ell \leq k} \hbar^{3-\frac{3}{2} k+(1-\alpha)(k-\ell) s} \int \cdots /(\mathrm{d} q \mathrm{~d} w \mathrm{~d} u)^{\otimes k}\left(\chi_{\left(w_{1}-u_{1}\right) \in \Omega_{\hbar}} \cdots \chi_{\left(w_{\ell}-u_{\ell}\right) \in \Omega_{\hbar}}\right) \\
& \cdot \prod_{n=1}^{k}\left|f\left(\frac{w_{n}-q_{n}}{\sqrt{\hbar}}\right) f\left(\frac{u_{n}-q_{n}}{\sqrt{\hbar}}\right)\right| \chi_{\left|w_{n}-q_{n}\right| \leq \sqrt{\hbar} R} \chi_{\left|u_{n}-q_{n}\right| \leq \sqrt{\hbar} R} \\
& \left\|a_{w_{k}} \cdots a_{w_{1}} \psi_{N, t}\right\|\left\|a_{u_{k}} \cdots a_{u_{1}} \psi_{N, t}\right\| \\
\leq & C \max _{0 \leq \ell \leq k} \hbar^{3-\frac{3}{2} k+(1-\alpha)(k-\ell) s} \int \cdots /(\mathrm{d} q)^{\otimes k}\left[\int(\mathrm{d} w \mathrm{~d} u)^{\otimes k}\left(\chi_{\left(w_{1}-u_{1}\right) \in \Omega_{\hbar}} \cdots \chi_{\left(w_{\ell}-u_{\ell}\right) \in \Omega_{\hbar}}\right)\right. \\
& \left.\cdot \prod_{n=1}^{k}\left|f\left(\frac{w_{n}-q_{n}}{\sqrt{\hbar}}\right) f\left(\frac{u_{n}-q_{n}}{\sqrt{\hbar}}\right)\right|^{2}\right]^{\frac{1}{2}}
\end{aligned}
$$




$$
\begin{aligned}
& {\left[\int \cdots /(\mathrm{d} w)^{\otimes k} \prod_{n=1}^{k} \chi_{\left|w_{n}-q_{n}\right| \leq \sqrt{\hbar} R}\left\|a_{w_{k}} \cdots a_{w_{1}} \psi_{N, t}\right\|^{2}\right] } \\
= & C \max _{0 \leq \ell \leq k} \hbar^{3+(1-\alpha)(k-\ell) s} \int \cdots /(\mathrm{d} q)^{\otimes k} \\
& {\left[\int \cdots \int(\mathrm{d} \widetilde{w} \mathrm{~d} \tilde{u})^{\otimes k}\left(\chi_{\left|\widetilde{w}_{1}-\widetilde{u}_{1}\right| \leq \hbar^{\alpha+\frac{1}{2}}} \cdots \chi_{\left|\widetilde{w}_{\ell}-\widetilde{u}_{\ell}\right| \leq \hbar^{\alpha+\frac{1}{2}}}\right)\right.} \\
& \left.\cdot \prod_{n=1}^{k}\left|f\left(\widetilde{w}_{n}\right) f\left(\tilde{u}_{n}\right)\right|^{2}\right]^{\frac{1}{2}} \\
& {\left[\int_{\cdots}(\mathrm{d} w)^{\otimes k} \prod_{n=1}^{k} \chi_{\left|w_{n}-q_{n}\right| \leq \sqrt{\hbar} R}\left\|a_{w_{k}} \cdots a_{w_{1}} \psi_{N, t}\right\|^{2}\right] } \\
\leq & C \max _{0 \leq \ell \leq k} \hbar^{3-\frac{3}{2} k+(1-\alpha)(k-\ell) s+\left(\alpha+\frac{1}{2}\right) \ell},
\end{aligned}
$$

where, as in the proof of Proposition 2.4, we applied Lemma 2.4 and (3.30). Therefore, we obtain the desired result by choosing $s=\left\lceil\frac{1+2 \alpha}{2(1-\alpha)}\right\rceil$.

Next, we switch to estimate (2.22) for $\widetilde{\mathcal{R}}_{k}$. Repeated the steps in the proof of Proposition 2.5, we have

$$
\begin{aligned}
\left|\int \ldots \int(\mathrm{d} q \mathrm{~d} p)^{\otimes k} \nabla_{\mathbf{p}_{k}} \Phi\left(q_{1}, p_{1}, \ldots, q_{k}, p_{k}\right) \cdot \widetilde{\mathcal{R}}_{k}\right| \\
=\mid \sum_{j=1}^{k} \int \cdots \int(\mathrm{d} q \mathrm{~d} p)^{\otimes k}(\mathrm{~d} w \mathrm{~d} u)^{\otimes k} \nabla_{p_{j}} \Phi\left(q_{1}, p_{1}, \ldots, q_{k}, p_{k}\right) \cdot \iint \mathrm{d} y \mathrm{~d} v \iint \mathrm{d} q_{k+1} \mathrm{~d} p_{k+1} \\
\quad \cdot \int_{0}^{1} \mathrm{~d} s\left[\nabla V\left(s u_{j}+(1-s) w_{j}-y\right)-\nabla V\left(q_{j}-y\right)+\nabla V\left(q_{j}-y\right)-\nabla V\left(q_{j}-q_{k+1}\right)\right] \\
\quad \cdot\left(f_{q, p}^{\hbar}(w) \overline{f_{q, p}^{\hbar}(u)}\right)^{\otimes k} f_{q_{k+1}, p_{k+1}}^{\hbar}(y) \overline{f_{q_{k+1}, p_{k+1}}^{\hbar}(v)}\left\langle a_{w_{k}} \cdots a_{w_{1}} a_{y} \psi_{N, t}, a_{u_{k}} \cdots a_{u_{1}} a_{v} \psi_{N, t}\right\rangle \mid .
\end{aligned}
$$

Appling the $\hbar$-weighted Dirac-delta function as in (2.7), we have

$$
\begin{aligned}
= & (2 \pi)^{3} \hbar^{3-\frac{3}{2}} \mid \sum_{j=1}^{k} \int \ldots \int(\mathrm{d} q \mathrm{~d} p)^{\otimes k}(\mathrm{~d} w \mathrm{~d} u)^{\otimes k} \nabla_{p_{j}} \Phi\left(q_{1}, p_{1}, \ldots, q_{k}, p_{k}\right) \cdot \iint \mathrm{d} y \mathrm{~d} q_{k+1} \\
& \cdot \int_{0}^{1} \mathrm{~d} s\left[\nabla V\left(s u_{j}+(1-s) w_{j}-y\right)-\nabla V\left(q_{j}-y\right)+\nabla V\left(q_{j}-y\right)-\nabla V\left(q_{j}-q_{k+1}\right)\right] \\
& \cdot\left(f_{q, p}^{\hbar}(w) \overline{f_{q, p}^{\hbar}(u)}\right){ }^{\otimes k}\left|f\left(\frac{y-q_{k+1}}{\sqrt{\hbar}}\right)\right|^{2}\left\langle a_{w_{k}} \cdots a_{w_{1}} a_{y} \psi_{N, t}, a_{u_{k}} \cdots a_{u_{1}} a_{y} \psi_{N, t}\right\rangle \mid \\
\leq & (2 \pi)^{3} \hbar^{3-\frac{3}{2} k} \sum_{j=1}^{k} \int \ldots \int(\mathrm{d} q \mathrm{~d} w \mathrm{~d} u)^{\otimes k} \prod_{n=1}^{k}\left|\int \ldots \int(\mathrm{d} p)^{\otimes k} \nabla_{p_{j}} \Phi\left(q_{1}, p_{1}, \ldots, q_{k}, p_{k}\right) e^{\frac{1}{\hbar} p_{n} \cdot\left(w_{n}-u_{n}\right)}\right| \\
& \iint_{\mathrm{d} y \mathrm{~d} \widetilde{q}_{k+1}} \\
& \cdot\left(\int_{0}^{1} \mathrm{~d} s \mid \nabla V\left(s u_{j}+(1-s) w_{j}-y\right)-\nabla V\left(q_{j}-y\right)\right) \mid \\
& \left.+\left|\nabla V\left(q_{j}-y\right)-\nabla V\left(q_{j}-y+\sqrt{\hbar} \widetilde{q}_{k+1}\right)\right|\right)
\end{aligned}
$$




$$
\left|f\left(\frac{w_{n}-q_{n}}{\sqrt{\hbar}}\right) f\left(\frac{u_{n}-q_{n}}{\sqrt{\hbar}}\right)\right|\left|f\left(\widetilde{q}_{k+1}\right)\right|^{2}\left|\left\langle a_{w_{k}} \cdots a_{w_{1}} a_{y} \psi_{N, t}, a_{u_{k}} \cdots a_{u_{1}} a_{y} \psi_{N, t}\right\rangle\right| .
$$

Using the fact that $\nabla V$ is Lipchitz continuous and that $f$ is compactly supported, we have

$$
\begin{aligned}
\leq & (2 \pi)^{3} \hbar^{3-\frac{3}{2} k} \sum_{j=1}^{k} \int \ldots \int(\mathrm{d} q \mathrm{~d} w \mathrm{~d} u)^{\otimes k} \prod_{n=1}^{k}\left|\int \ldots \int(\mathrm{d} p)^{\otimes k} \nabla_{p_{j}} \Phi\left(q_{1}, p_{1}, \ldots, q_{k}, p_{k}\right) e^{\frac{\mathrm{i}}{\hbar} p_{n} \cdot\left(w_{n}-u_{n}\right)}\right| \\
& \iint \mathrm{d} y \mathrm{~d} \widetilde{q}_{k+1} \cdot\left(\int_{0}^{1} \mathrm{~d} s\left|s u_{j}+(1-s) w_{j}-q_{j}\right|+\left|\sqrt{\hbar} \widetilde{q}_{k+1}\right|\right) \\
& \left|f\left(\frac{w_{n}-q_{n}}{\sqrt{\hbar}}\right) f\left(\frac{u_{n}-q_{n}}{\sqrt{\hbar}}\right)\right|\left|f\left(\widetilde{q}_{k+1}\right)\right|^{2} \\
& \cdot \chi_{\left|w_{n}-q_{n}\right| \leq \sqrt{\hbar} R} \chi_{\left|u_{n}-q_{n}\right| \leq \sqrt{\hbar} R} \mid\left\|a_{w_{k}} \cdots a_{w_{1}} a_{y} \psi_{N, t}\right\|\left\|a_{u_{k}} \cdots a_{u_{1}} a_{y} \psi_{N, t}\right\| \\
= & I_{4}+I I_{4}
\end{aligned}
$$

Focusing on $I_{4}$, we split the integral as follows

$$
\begin{aligned}
I_{4}= & (2 \pi)^{3} \hbar^{3-\frac{3}{2} k} \sum_{j=1}^{k} \int \ldots \int(\mathrm{d} q \mathrm{~d} w \mathrm{~d} u)^{\otimes k} \mid \prod_{n=1}^{k}\left(\chi_{\left(w_{n}-u_{n}\right) \in \Omega_{\hbar}^{c}}+\chi_{\left(w_{n}-u_{n}\right) \in \Omega_{\hbar}}\right) \\
& \int \ldots \int(\mathrm{d} p)^{\otimes k} \nabla_{p_{j}} \Phi\left(q_{1}, p_{1}, \ldots, q_{k}, p_{k}\right) \\
& \cdot e^{\frac{i}{\hbar} \sum_{m=1}^{k} p_{m} \cdot\left(w_{m}-u_{m}\right)} \mid \\
& \left.\iint \mathrm{d} y \mathrm{~d} \widetilde{q}_{k+1} \int_{0}^{1} \mathrm{~d} s\left|s u_{j}+(1-s) w_{j}-q_{j}\right|\left|f\left(\frac{w_{n}-q_{n}}{\sqrt{\hbar}}\right) f\left(\frac{u_{n}-q_{n}}{\sqrt{\hbar}}\right)\right| f\left(\widetilde{q}_{k+1}\right)\right|^{2} \\
& \cdot \chi_{\left|w_{n}-q_{n}\right| \leq \sqrt{\hbar} R} \chi_{\left|u_{n}-q_{n}\right| \leq \sqrt{\hbar} R} \mid\left\|a_{w_{k}} \cdots a_{w_{1}} a_{y} \psi_{N, t}\right\|\left\|a_{u_{k}} \cdots a_{u_{1}} a_{y} \psi_{N, t}\right\| .
\end{aligned}
$$

where $\Omega_{\hbar}$ is defined as in (2.10). We do similar computations for $I I_{4}$,

$$
\begin{aligned}
I I_{4}=(2 \pi)^{3} \hbar^{3-\frac{3}{2} k} \sum_{j=1}^{k} \int \ldots \int(\mathrm{d} q \mathrm{~d} w \mathrm{~d} u)^{\otimes k} \mid \prod_{n=1}^{k} \int \ldots \int(\mathrm{d} p)^{\otimes k}\left(\chi_{\left(w_{n}-u_{n}\right) \in \Omega_{\hbar}^{c}}+\chi_{\left(w_{n}-u_{n}\right) \in \Omega_{\hbar}}\right) \\
\quad \nabla_{p_{j}} \Phi\left(q_{1}, p_{1}, \ldots, q_{k}, p_{k}\right) \\
\left.\quad \cdot e^{\frac{1}{\hbar} p_{n} \cdot\left(w_{n}-u_{n}\right)}\left|\iint \mathrm{d} y \mathrm{~d} \widetilde{q}_{k+1}\right| \sqrt{\hbar} \widetilde{q}_{k+1}|| f\left(\frac{w_{n}-q_{n}}{\sqrt{\hbar}}\right) f\left(\frac{u_{n}-q_{n}}{\sqrt{\hbar}}\right)|| f\left(\widetilde{q}_{k+1}\right)\right|^{2} \\
\cdot \chi_{\left|w_{n}-q_{n}\right| \leq \sqrt{\hbar} R} \chi_{\left|u_{n}-q_{n}\right| \leq \sqrt{\hbar} R} \mid\left\|a_{w_{k}} \cdots a_{w_{1}} a_{y} \psi_{N, t}\right\|\left\|a_{u_{k}} \cdots a_{u_{1}} a_{y} \psi_{N, t}\right\| .
\end{aligned}
$$

Repeating the proof of Proposition 2.5, namely in (3.36) and (3.32), as well as the proof for estimate (2.21), we eventually obtain

$$
I_{4}+I I_{4} \leq C \max _{0 \leq \ell \leq k} \hbar^{\frac{1}{2}-\frac{3}{2} k+(1-\alpha)(k-\ell) s+\left(\alpha+\frac{1}{2}\right) \ell},
$$

where the constant $C$ depends on $k$. As before, we choose $s=\left\lceil\frac{1+2 \alpha}{2(1-\alpha)}\right\rceil$ and choose $\alpha \in$ $\left(\frac{1}{2}, 1\right)$ such that $(\alpha-1) k \leq-\delta$, and we obtain the desired estimates.

Acknowledgements We are also grateful to the anonymous referee for carefully reading our manuscript and providing helpful comments. We acknowledge support by the Deutsche Forschungsgemeinschaft through the grants CH 955/4-1. Jinyeop Lee was partially supported by Samsung Science and Technology Foundation 
(SSTF-BA1401-51) and by the National Research Foundation of Korea(NRF) grant funded by the Korea government(MSIT) (NRF-2019R1A5A1028324 and NRF-2020R1F1A1A01070580).

Funding Open Access funding enabled and organized by Projekt DEAL.

Open Access This article is licensed under a Creative Commons Attribution 4.0 International License, which permits use, sharing, adaptation, distribution and reproduction in any medium or format, as long as you give appropriate credit to the original author(s) and the source, provide a link to the Creative Commons licence, and indicate if changes were made. The images or other third party material in this article are included in the article's Creative Commons licence, unless indicated otherwise in a credit line to the material. If material is not included in the article's Creative Commons licence and your intended use is not permitted by statutory regulation or exceeds the permitted use, you will need to obtain permission directly from the copyright holder. To view a copy of this licence, visit http://creativecommons.org/licenses/by/4.0/.

\section{References}

1. Amour, L., Khodja, M., Nourrigat, J.: The classical limit of the Heisenberg and time-dependent HartreeFock equations: the Wick symbol of the solution. Math. Res. Lett. 20(1), 119-139 (2013)

2. Amour, L., Khodja, M., Nourrigat, J.: The semiclassical limit of the time dependent Hartree-Fock equation: the Weyl symbol of the solution. Anal. PDE 6(7), 1649-1674 (2013)

3. Bach, V., Breteaux, S., Petrat, S., Pickl, P., Tzaneteas, T.: Kinetic energy estimates for the accuracy of the time-dependent Hartree-Fock approximation with Coulomb interaction. J. Math. Pures Appl. 105(1), 1-30 (2016)

4. Benedikter, N., Jakšić, V., Porta, M., Saffirio, C., Schlein, B.: Mean-field evolution of fermionic mixed states. Commun. Pure Appl. Math. 69(12), 2250-2303 (2016)

5. Benedikter, N., Porta, M., Saffirio, C., Schlein, B.: From the Hartree dynamics to the Vlasov equation. Arch. Ration. Mech. Anal. 221(1), 273-334 (2016)

6. Benedikter, N., Porta, M., Schlein, B.: Mean-field evolution of fermionic systems. Commun. Math. Phys. 331(3), 1087-1131 (2014)

7. Benedikter, N., Porta, M., Schlein, B.: Mean-field dynamics of fermions with relativistic dispersion. J. Math. Phys. 55(2), 021901 (2014)

8. Benedikter, N., Porta, M., Schlein, B.: Effective Evolution Equations from Quantum Dynamics, vol. 7. Springer, New York (2016)

9. Bröcker, T., Werner, R.F.: Mixed states with positive Wigner functions. J. Math. Phys. 36(1), 62-75 (1995)

10. Chen, L., Lee, J.O., Lee, J.: Rate of convergence toward Hartree dynamics with singular interaction potential. J. Math. Phys. 59(3), 031902 (2018)

11. Chen, L., Lee, J.O., Schlein, B.: Rate of convergence towards Hartree dynamics. J. Stat. Phys. 144(4), 872 (2011)

12. Dell'Antonio, G.: Lectures on the Mathematics of Quantum Mechanics II: Selected Topics. Springer, New York (2016)

13. Diestel, J.: Uniform integrability: an introduction. Rend. Istit. Mat. Univ. Trieste 23(1), 41-80 (1993) (1991). School on Measure Theory and Real Analysis (Grado, 1991)

14. Dietler, E., Rademacher, S., Schlein, B.: From Hartree dynamics to the relativistic Vlasov equation. J. Stat. Phys. 172(2), 398-433 (2018)

15. Dobrushin, R.L.: Vlasov equations. Funct. Anal. Appl. 13(2), 115-123 (1979)

16. Elgart, A., Erdős, L., Schlein, B., Yau, H.T.: Nonlinear Hartree equation as the mean field limit of weakly coupled fermions. J. Math. Pures Appl. 83(10), 1241-1273 (2004)

17. Erdos, L., Yau, H.T.: Derivation of the nonlinear Schrödinger equation with Coulomb potential. Tech. rep. (2001)

18. Fefferman, C., de la Llave, R.: Relativistic stability of matter-i. Revista Matematica Iberoamericana 2(2), 119-213 (1986)

19. Fournais, S., Lewin, M., Solovej, J.P.: The semi-classical limit of large fermionic systems. Calc. Var. Partial. Differ. Equ. 57(4), 105 (2018)

20. Fröhlich, J., Knowles, A.: A microscopic derivation of the time-dependent Hartree-Fock equation with Coulomb two-body interaction. J. Stat. Phys. 145(1), 23 (2011)

21. Gasser, I., Illner, R., Markowich, P.A., Schmeiser, C.: Semiclassical, $t \rightarrow \infty$ asymptotics and dispersive effects for Hartree-Fock systems. ESAIM: Mathematical Modelling and Numerical Analysis - Modélisation Mathématique et Analyse Numérique 32(6), 699-713 (1998) 
22. Golse, F., Mouhot, C., Paul, T.: On the mean field and classical limits of quantum mechanics. Commun. Math. Phys. 343(1), 165-205 (2016)

23. Golse, F., Paul, T.: The Schrödinger equation in the mean-field and semiclassical regime. Arch. Ration. Mech. Anal. 223(1), 57-94 (2017)

24. Golse, F., Paul, T.: Empirical measures and quantum mechanics: applications to the mean-field limit. Commun. Math. Phys. 369(3), 1021-1053 (2019)

25. Golse, F., Paul, T., Pulvirenti, M.: On the derivation of the Hartree equation in the mean field limit: uniformity in the Planck constant. J. Funct. Anal. 275(7), 1603-1649 (2018)

26. Hainzl, C., Seiringer, R.: General decomposition of radial functions on $R^{n}$ and applications to $N$-body quantum systems. Lett. Math. Phys. 61(1), 75-84 (2002)

27. Hudson, R.: When is the Wigner quasi-probability density non-negative? Rep. Math. Phys. 6(2), 249-252 (1974)

28. Lafleche, L.: Global semiclassical limit from Hartree to Vlasov equation for concentrated initial data. arXiv preprint arXiv:1902.08520 (2019)

29. Lafleche, L.: Propagation of moments and semiclassical limit from Hartree to Vlasov equation. J. Stat. Phys. 177(1), 20-60 (2019)

30. Laflèche, L., Saffirio, C.: Strong semiclassical limit from Hartree and Hartree-Fock to Vlasov-Poisson equation. arXiv preprint arXiv:2003.02926 (2020)

31. Lions, P.L., Paul, T.: Sur les mesures de Wigner. Revista Matemática Iberoamericana 9(3), 553-618 (1993)

32. Mandilara, A., Karpov, E., Cerf, N.J.: Extending Hudson's theorem to mixed quantum states. Phys. Rev. A 79, 062302 (2009)

33. Markowich, P.A., Mauser, N.J.: The classical limit of a self-consistent quantum-Vlasov equation in $3 \mathrm{~d}$. Math. Models Methods Appl. Sci. 3, 109-124 (1993)

34. Narnhofer, H., Sewell, G.L.: Vlasov hydrodynamics of a quantum mechanical model. Commun. Math. Phys. 79(1), 9-24 (1981)

35. Petrat, S.: Derivation of mean-field dynamics for fermions. Ph.D. thesis (2014)

36. Petrat, S.: Hartree corrections in a mean-field limit for fermions with Coulomb interaction. J. Phys. A 50(24), 244004 (2017)

37. Petrat, S., Pickl, P.: A new method and a new scaling for deriving fermionic mean-field dynamics. Math. Phys. Anal. Geom. 19, 1-51 (2016)

38. Porta, M., Rademacher, S., Saffirio, C., Schlein, B.: Mean field evolution of fermions with coulomb interaction. J. Stat. Phys. 166(6), 1345-1364 (2017)

39. Robert, M.C.D.: Coherent States and Applications in Mathematical Physics. Springer, Netherlands (2012)

40. Rodnianski, I., Schlein, B.: Quantum fluctuations and rate of convergence towards mean field dynamics. Commun. Math. Phys. 291(1), 31-61 (2009)

41. Saffirio, C.: Mean-field evolution of fermions with singular interaction. In: Workshop on Macroscopic Limits of Quantum Systems, pp. 81-99. Springer (2017)

42. Saffirio, C.: From the Hartree equation to the Vlasov-Poisson system: strong convergence for a class of mixed states. arXiv preprint arXiv:1903.06013 (2019)

43. Saffirio, C.: Semiclassical limit to the Vlasov equation with inverse power law potentials. Commun. Math. Phys. 373(2), 571-619 (2020)

44. Santambrogio, F.: Optimal Transport for Applied Mathematicians. Springer, New York (2015)

45. Soto, F., Claverie, P.: When is the Wigner function of multidimensional systems nonnegative? J. Math. Phys. 24(1), 97-100 (1983)

46. Spohn, H.: On the Vlasov hierarchy. Math. Methods Appl. Sci. 3(1), 445-455 (1981)

47. Villani, C.: Topics in Optimal Transportation. American Mathematical Society, Providence (2003)

48. Zhang, P.: Wigner Measure and Semiclassical Limits of Nonlinear Schrödinger Equations. American Mathematical Society, Providence (2008)

Publisher's Note Springer Nature remains neutral with regard to jurisdictional claims in published maps and institutional affiliations. 